Pacific

Journal of

Mathematics

\title{
LE FLOT GÉODÉSIQUE DES QUOTIENTS GÉOMÉTRIQUEMENT FINIS DES GÉOMÉTRIES DE HILBERT
}

MickAëL CRAMPON ET LudoviC MARQUiS 


\title{
LE FLOT GÉODÉSIQUE DES QUOTIENTS GÉOMÉTRIQUEMENT FINIS DES GÉOMÉTRIES DE HILBERT
}

\author{
MickaËl CRAMPON ET LudoviC MARQuis
}

On étudie le flot géodésique des quotients géométriquement finis $\Omega / \Gamma$ de géométries de Hilbert, en particulier ses propriétés de récurrence.

On prouve, sous une hypothèse géométrique sur les pointes, que le flot géodésique est uniformément hyperbolique. Sans cette hypothèse, on construit un exemple où celui-ci a un exposant de Lyapunov nul.

On fait le lien entre la dynamique du flot géodésique et certaines propriétés du convexe $\Omega$ et du groupe $\Gamma$. On en déduit des résultats de rigidité, qui étendent ceux de Benoist et Guichard pour les quotients compacts.

Enfin, on s'intéresse au lien entre entropie volumique et exposant critique; on montre entre autres qu'ils coïncident lorsque le quotient est de volume fini.

We study the geodesic flow of geometrically finite quotients $\Omega$ / $\Gamma$ of Hilbert geometries, in particular its recurrence properties.

We prove that, under a geometric assumption on the cusps, the geodesic flow is uniformly hyperbolic. Without this assumption, we provide an example of a quotient whose geodesic flow has a zero Lyapunov exponent.

We make the link between the dynamics of the geodesic flow and some properties of the convex set $\Omega$ and the group $\Gamma$. As a consequence, we get various rigidity results which extend previous results of Benoist and Guichard for compact quotients.

Finally, we study the link between volume entropy and critical exponent; for example, we show that they coincide provided the quotient has finite volume.

1. Introduction

2. Géométries de Hilbert 320

3. Le flot géodésique 326

4. Variétés géométriquement finies 330

5. Hyperbolicité uniforme du flot géodésique 335

6. Propriétés de récurrence 340

7. Régularité du bord 343

MSC2010: 20H10, 22E40, 37D40, 53A20.

Mots-clefs: Hilbert geometry, geodesic flow, geometrically finite, volume entropy. 
8. Quelques exemples 354

9. Entropie volumique et exposant critique 358

Remerciements 368

Bibliographie 368

\section{Introduction}

Cet article dynamique fait logiquement suite à l'article géométrique [Crampon et Marquis 2012], dans lequel nous étudions la notion de finitude géométrique en géométrie de Hilbert. Avec [Crampon et Marquis 2013], ils forment un seul travail que nous avons découpé en trois pour des raisons évidentes de longueur. Concernant la géométrie des variétés géométriquement finies, nous ne rappellerons dans ce texte que les résultats dont nous ferons usage et renvoyons le lecteur à [Crampon et Marquis 2012] pour plus d'informations.

Une géométrie de Hilbert est un espace métrique $\left(\Omega, d_{\Omega}\right)$ où $\Omega$ est un ouvert proprement convexe de l'espace projectif réel $\mathbb{P}^{n}=\mathbb{P}^{n}(\mathbb{R})$ et $d_{\Omega}$ est la distance définie sur $\Omega$ par

$$
d_{\Omega}(x, y)=\frac{1}{2}|\ln ([p: q: x: y])|, \quad x, y \in \Omega \text { distincts; }
$$

dans cette formule, les points $p$ et $q$ sont les points d'intersection de la droite ( $x y)$ avec le bord $\partial \Omega$ de $\Omega$. Ces géométries ont été introduites par Hilbert comme exemples de géométries dans lesquelles les droites sont des géodésiques. Leur définition imite celle de l'espace hyperbolique dans le modèle projectif de Beltrami, qui correspond à la géométrie de Hilbert définie par un ellipsoïde.

Lorsque l'ouvert convexe $\Omega$ est strictement convexe, la géométrie de Hilbert est uniquement géodésique : les droites sont les seules géodésiques. On peut dans ce cas définir le flot géodésique sans recourir à des équations géodésiques, comme on le fait de façon traditionnelle en géométrie riemannienne. Le flot géodésique est ainsi le flot défini sur le fibré homogène $H \Omega=T \Omega \backslash\{0\} / \mathbb{R}^{+}$de la façon suivante :

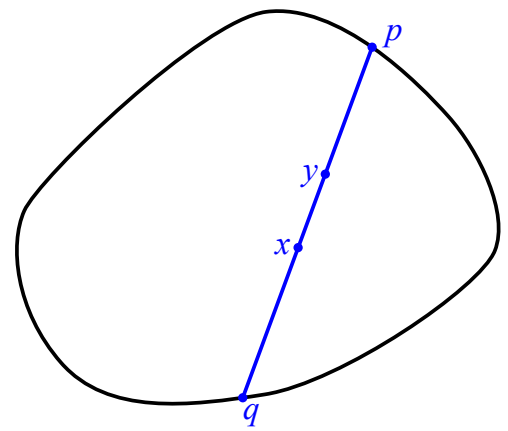

Figure 1. La distance de Hilbert. 
si $w=(x,[\xi])$ est un point de $H \Omega$, consistant en un point $x$ de $\Omega$ et une direction tangente $[\xi]$, on trouve son image $\varphi^{t}(w)$ en suivant la droite géodésique partant de $x$ dans la direction [ $\xi]$.

Les géométries de Hilbert sont des espaces finslériens : la métrique de Hilbert est engendrée par un champ de normes $F: T \Omega \rightarrow \mathbb{R}$ sur $\Omega$, donné par la formule

$$
F(x, \xi)=\frac{|\xi|}{2}\left(\frac{1}{\left|x x^{-}\right|}+\frac{1}{\left|x x^{+}\right|}\right), \quad(x, \xi) \in T \Omega,
$$

où $x^{+}$et $x^{-}$sont les points d'intersection de la droite $\{x+\lambda \xi \mid \lambda \in \mathbb{R}\}$ avec $\partial \Omega$ (voir le paragraphe $2 \mathrm{~A}$ pour plus de précisions). Lorsque $\partial \Omega$ est de classe $\mathscr{C}^{2}$ à hessien défini positif, alors on peut définir les géodésiques au moyen d'une équation différentielle, et le flot géodésique est le flot de cette équation.

La géométrie de Hilbert définie par un ouvert strictement convexe à bord $\mathscr{C}^{1}$ possède un certain comportement hyperbolique. Par exemple, dans ce cadre-là, on voit apparaître naturellement, au moyen des horosphères, les variétés stables et instables du flot géodésique. Le flot géodésique est dans ce cas de classe $\mathscr{C}^{1}$ et l'espace tangent à $H \Omega$ admet une décomposition en

$$
T H \Omega=\mathbb{R} \cdot X \oplus E^{s} \oplus E^{u},
$$

où $X$ est le générateur du flot, $E^{s}$ est la distribution stable tangente au feuilletage stable, et $E^{u}$ est la distribution instable.

Les flots géodésiques des variétés riemanniennes compactes de courbure négative sont les premiers exemples de flots d'Anosov, ou uniformément hyperboliques. Cette propriété d'hyperbolicité ne dépend que des bornes sur la courbure et elle reste donc vraie pour une variété riemannienne non compacte à courbure négative $K<-a^{2}<0$.

Pour une géométrie de Hilbert quelconque, on ne peut espérer obtenir de propriété d'hyperbolicité. En effet, le comportement asymptotique autour d'une géodésique dépend de la régularité du bord du convexe au point extrémal de la géodésique (voir [Crampon 2014] pour une étude détaillée). Par contre, si la géométrie admet un quotient assez petit, on peut s'attendre à des propriétés de récurrence sur le quotient.

C'est le cas lorsqu'il existe un quotient compact : dans [Benoist 2004], Yves Benoist a prouvé que le flot géodésique d'un quotient compact d'une géométrie de Hilbert (avec $\Omega$ strictement convexe) était un flot d'Anosov. Notre premier théorème généralise cela au flot géodésique de certaines variétés géométriquement finies.

Les variétés géométriquement finies sont en quelque sorte les variétés non compactes les plus simples. Leur caractéristique essentielle pour nous est que leur cœur convexe se décompose en une partie compacte et un nombre fini de pointes (cusps, en anglais). C'est essentiel car le cœur convexe est le support de l'ensemble non 
errant du flot géodésique; c'est donc là que se concentre la dynamique. On renvoie au fait 3 ou à l'article [Crampon et Marquis 2012] pour plus de détails.

À chaque fois, on va essayer de comprendre séparément ce qu'il se passe sur la partie compacte puis sur les parties cuspidales. De façon générale, on ne peut rien dire sans faire d'hypothèses sur la géométrie des pointes :

Proposition 1.1 (proposition 8.1). Il existe une variété géométriquement finie $M=\Omega / \Gamma$ dont le flot géodésique a un exposant de Lyapunov nul. En particulier, le flot géodésique n'est pas uniformément hyperbolique.

Dans ce texte, nous étudierons donc principalement les variétés géométriquement finies dont les pointes sont «asymptotiquement hyperboliques » : dans une pointe, la métrique de Hilbert est équivalente à une métrique hyperbolique qui a les mêmes géodésiques (non paramétrées); voir la définition 4.4. Parmi les variétés géométriquement finies à pointes asymptotiquement hyperboliques, on trouve en particulier les variétés de volume fini, et plus généralement celles dont les sous-groupes paraboliques maximaux sont de rang maximal, c'est-à-dire qu'ils agissent cocompactement sur $\partial \Omega \backslash\{p\}$, où $p$ est le point fixe du groupe parabolique considéré.

Il est fort possible que pour toute variété géométriquement finie $M=\Omega / \Gamma$, il existe un ouvert $\Omega^{\prime}, \Gamma$-invariant, strictement convexe et à bord $\mathscr{C}^{1}$, tel que le quotient $M^{\prime}=\Omega^{\prime} / \Gamma$ est géométriquement fini à pointes asymptotiquement hyperboliques. La raison principale qui nous pousse à penser qu'une telle construction est possible est que les sous-groupes paraboliques d'un tel groupe $\Gamma$ sont conjugués à des sous-groupes paraboliques de $\mathrm{SO}_{n, 1}(\mathbb{R})$.

Pour ces variétés-là, on peut prouver ceci :

Théorème 1.2 (théorème 5.2). Soient $\Omega$ un ouvert strictement convexe et à bord $\mathscr{C}^{1}$, et $M=\Omega / \Gamma$ une variété géométriquement finie à pointes asymptotiquement hyperboliques. Le flot géodésique de la métrique de Hilbert est uniformément hyperbolique sur son ensemble non errant $\mathrm{NW}$ : le fibré tangent à $H M$ admet en tout point de NW une décomposition $\varphi^{t}$-invariante

$$
T H M=\mathbb{R} \cdot X \oplus E^{s} \oplus E^{u},
$$

telle qu'il existe des constantes $\chi, C>0$ pour lesquelles

$$
\text { (1-1) }\left\|d \varphi^{t} Z^{s}\right\| \leqslant C e^{-\chi t} \quad \text { et }\left\|d \varphi^{-t} Z^{u}\right\| \leqslant C e^{-\chi t}, \quad Z^{s} \in E^{s}, Z^{u} \in E^{u}, t \geqslant 0 .
$$

De façon générale, on prouvera aussi les propriétés de récurrence suivantes :

Proposition 1.3 (proposition 6.1). Soient $\Omega$ un ouvert strictement convexe et à bord $\mathscr{C}^{1}$, et $M=\Omega / \Gamma$ une variété quotient. Le flot géodésique de $M$ est topologiquement mélangeant sur son ensemble non errant. 
Notre deuxième théorème concerne la régularité du bord des ouverts convexes $\Omega$ qui admettent un quotient géométriquement fini $M=\Omega / \Gamma$ non compact. Ce résultat est lié au fait que les propriétés hyperboliques des orbites du flot géodésique se lisent directement sur la régularité du bord au niveau de leur point extrémal.

Bien entendu, cela permet de décrire le bord uniquement au niveau de l'ensemble limite $\Lambda_{\Gamma}$ du groupe. Ce n'est pas étonnant puisque celui-ci constitue l'ensemble des points extrémaux des géodésiques récurrentes. De plus, c'est la seule partie du bord qui est imposée par le groupe $\Gamma$ : on peut en effet modifier le bord (presque) à sa guise hors de l'ensemble limite; c'est d'ailleurs ainsi qu'on obtient l'exemple de la proposition 1.1. Pour un quotient compact ou de volume fini, l'ensemble limite est le bord tout entier et donc le convexe $\Omega$ est entièrement déterminé par le groupe $\Gamma$.

Théorème 1.4 (corollaire 7.3). Soient $\Omega$ un ouvert strictement convexe et à bord $\mathscr{C}^{1}$, et $M=\Omega / \Gamma$ une variété géométriquement finie à pointes asymptotiquement hyperboliques. Il existe $\varepsilon>0$ tel que le bord $\partial \Omega$ du convexe $\Omega$ est de classe $\mathscr{C}^{1+\varepsilon}$ en tout point de $\Lambda_{\Gamma}$.

Via la caractérisation des quotients de volume fini par leur ensemble limite, on obtient le corollaire suivant.

Corollaire 1.5 (corollaire 7.3). Soit $\Omega$ un ouvert strictement convexe et à bord $\mathfrak{b}^{1}$. Si $\Omega$ admet un quotient de volume fini, alors son bord $\partial \Omega$ est de classe $\varphi^{1+\varepsilon}$ pour un certain $\varepsilon>0$.

Lorsque $\Omega$ admet un quotient compact, Olivier Guichard a pu déterminer exactement la régularité optimale du bord, c'est-à-dire le plus grand $\varepsilon$ tel que le bord $\partial \Omega$ est $\mathscr{C}^{1+\varepsilon}$. Celle-ci est encore une fois déterminée par le groupe $\Gamma$, via les valeurs propres de ses éléments hyperboliques. Cela n'est pas étonnant, étant donné que les orbites périodiques sont denses, et que celles-ci sont en bijection avec les classes de conjugaison d'éléments hyperboliques de $\Gamma$.

Si on se restreint à l'ensemble limite et l'ensemble non errant, cette observation reste valable pour un quotient quelconque. Nous pouvons ainsi prouver un résultat similaire pour les ouverts convexes qui admettent un quotient géométriquement fini à pointes asymptotiquement hyperboliques. Pour l'énoncé, définissons d'abord

$$
\varepsilon\left(\Lambda_{\Gamma}\right)=\sup \left\{\varepsilon \in[0,1] \mid \text { le bord } \partial \Omega \text { est } \mathscr{C}^{1+\varepsilon} \text { en tout point de } \Lambda_{\Gamma}\right\} .
$$

En suite, pour tout élément hyperbolique $\gamma \in \Gamma$, notons

$$
\varepsilon(\gamma)=\sup \left\{\varepsilon \in[0,1] \mid \text { le bord } \partial \Omega \text { est } \mathscr{C}^{1+\varepsilon} \text { au point attractif } x_{\gamma}^{+} \text {de } \gamma\right\},
$$

et $\varepsilon(\Gamma)=\inf \{\varepsilon(\gamma) \mid \gamma \in \Gamma$ hyperbolique $\}$. Ainsi, le bord $\partial \Omega$ est $\mathscr{C}^{1+\varepsilon(\Gamma)}$ en tout point fixe hyperbolique. On obtient alors : 
Théorème 1.6 (théorème 7.4). Soient $\Omega$ un ouvert strictement convexe et à bord $\mathscr{C}^{1}$, et $M=\Omega / \Gamma$ une variété géométriquement finie à pointes asymptotiquement hyperboliques. On a

$$
\varepsilon\left(\Lambda_{\Gamma}\right)=\varepsilon(\Gamma) .
$$

Notre démonstration de ce théorème est différente de celle de Guichard et repose sur l'extension d'un théorème de Ursula Hamenstädt [1994], qui s’intéresse au meilleur coefficient de contraction d'un flot uniformément hyperbolique.

Pour l'énoncer, il nous faut définir les meilleurs coefficients de contraction du flot sur l'ensemble non errant $\chi(\mathrm{NW})=\sup \{\chi \mid$ il existe $C>0$ tel que l'inégalité (1-1) a lieu en tout point de NW $\}$, et sur les orbites périodiques

$$
\chi(\operatorname{Per})=\inf \{\chi(w) \mid w \in \text { NW périodique }\} .
$$

Théorème 1.7 (théorème 7.5). Soient $\Omega$ un ouvert strictement convexe et à bord $\mathscr{C}^{1}$, et $M=\Omega / \Gamma$ une variété géométriquement finie à pointes asymptotiquement hyperboliques. On a

$$
\chi(\mathrm{Per})=\chi(\mathrm{NW}) .
$$

Comme corollaire de ces résultats et du travail précédent [Crampon et Marquis 2012], on obtient un résultat de rigidité :

Théorème 1.8 (corollaire 7.10). Soit $\Omega$ un ouvert strictement convexe et à bord $\mathscr{C}^{1}$, qui admet une action géométriquement finie d'un groupe $\Gamma$ contenant un élément parabolique. Si le bord $\partial \Omega$ est de classe $\mathscr{C}^{1+\varepsilon}$ pour tout $0<\varepsilon<1$, alors $\Gamma$ est un sous-groupe d'un conjugué de $\mathrm{SO}_{n, 1}(\mathbb{R})$.

Comme cas particulier, on obtient une version volume fini d'un théorème de Benoist [2004] qui concernait les quotients compacts :

Corollaire 1.9 (corollaire 7.11). Soit $\Omega$ un ouvert strictement convexe et à bord $\mathscr{C}^{1}$ qui admet un quotient de volume fini non compact. Si le bord $\partial \Omega$ est de classe $\mathscr{b}^{1+\varepsilon}$ pour tout $0<\varepsilon<1$, alors $\Omega$ est un ellipsoïde.

Remarquons que dans l'énoncé de ce théorème, tout comme dans celui du corollaire 1.5, l'une des hypothèses strictement convexe/à bord $\mathscr{C}^{1}$ est superflue : c'est une conséquence du travail de Daryl Cooper, Darren Long et Stephan Tillmann [Cooper et al. 2011].

À la fin de ce texte, on revient sur la représentation sphérique de $\mathrm{SL}_{2}(\mathbb{R})$ dans $\mathrm{SL}_{5}(\mathbb{R})$, que nous avions étudié dans [Crampon et Marquis 2012] car elle permettait de distinguer les deux notions de finitude géométrique que nous y avions introduites. En particulier, on avait vu que l'ensemble des ouverts proprement convexes préservés par cette représentation formait, à action de $\mathrm{SL}_{5}(\mathbb{R})$ près, une famille croissante 
$\left\{\Omega_{r} \mid 0 \leqslant r \leqslant \infty\right\}$. Parmi eux, les convexes $\Omega_{0}$ et $\Omega_{\infty}$, duaux l'un de l'autre, n'étaient ni strictement convexes ni à bord $\mathscr{C}^{1}$. Les autres par contre l'étaient. En fait, on peut déterminer précisément leur régularité (voir définition 4.5 pour les notions de régularité $\mathscr{C}^{1+\varepsilon}$ et la $\beta$-convexité) :

Proposition 1.10 (proposition 8.2). Pour $0<r<\infty$, le bord de l'ouvert convexe $\Omega_{r}$ est de classe $\mathscr{C}^{4 / 3}$ et 4 -convexe.

Nous avons inclus pour finir une première étude de la croissance des groupes discrets dont l'action est géométriquement finie sur $\Omega$. L'objet principal est l'exposant critique $\delta_{\Gamma}$ du groupe $\Gamma$, qui mesure la croissance exponentielle du groupe agissant sur $\Omega$; à savoir

$$
\delta_{\Gamma}=\limsup _{R \rightarrow+\infty} \frac{1}{R} \log \sharp\left\{g \in \Gamma \mid d_{\Omega}(x, g x) \leqslant R\right\} .
$$

Lorsque $\Gamma$ est un groupe cocompact, il est immédiat que l'exposant critique et l'entropie volumique de la géométrie de Hilbert sont égaux. Rappelons que l'entropie volumique de la géométrie de Hilbert $\left(\Omega, d_{\Omega}\right)$ est le taux de croissance exponentiel des volumes des boules :

$$
h_{\mathrm{vol}}(\Omega)=\limsup _{R \rightarrow+\infty} \frac{1}{R} \log \operatorname{Vol}_{\Omega} B(x, R) .
$$

Lorsque $\Gamma$ n'est plus cocompact, on a de façon générale $\delta_{\Gamma} \leqslant h_{\mathrm{vol}}$ mais il n'y a a priori plus de raisons pour que ces deux quantités coïncident, même si $\Gamma$ est de covolume fini : Françoise Dal'bo, Marc Peigné, Jean-Claude Picaud et Andrea Sambusetti ont construit des exemples de réseaux non uniformes d'espaces de courbure négative pincée où $\delta_{\Gamma}<h_{\mathrm{vol}}$.

Dans notre cas, le fait que les pointes d'une variété de volume fini soient asymptotiquement hyperboliques entraîne l'égalité :

Théorème 1.11 (théorème 9.2). Soient $\Omega$ un ouvert strictement convexe et à bord $\mathscr{C}^{1}$, et $\Gamma$ un sous-groupe discret de $\operatorname{Aut}(\Omega)$ de covolume fini. Alors

$$
\delta_{\Gamma}=h_{\mathrm{vol}}(\Omega) .
$$

Ce résultat peut même s'étendre au cas des actions géométriquement finies de la manière suivante :

Théorème 1.12 (théorème 9.7). Soit $\Gamma$ un sous-groupe discret de $\operatorname{Aut}(\Omega) d o n t$ l'action sur $\Omega$ est géométriquement finie. Alors

$$
\delta_{\Gamma}=\limsup _{R \rightarrow+\infty} \frac{1}{R} \log \operatorname{Vol}_{\Omega}\left(B(o, R) \cap C\left(\Lambda_{\Gamma}\right)\right),
$$

où o est un point quelconque de $\Omega$. 
Plan. Les sections 2 et 3 sont des préliminaires portant respectivement sur les géométries de Hilbert et leur flot géodésique.

La section 4 explique ce qui nous sera utile sur les variétés géométriquement finies, en présentant notamment l'hypothèse d'asymptoticité hyperbolique des pointes.

La section 5 est consacrée à la démonstration du théorème 1.2. Bien que l'idée soit claire et très simple, la démonstration reste malgré tout quelque peu technique.

La section 6 se concentre sur les propriétés de récurrence du flot géodésique d'une variété quelconque $M=\Omega / \Gamma$; en particulier, on y montre la proposition 1.3.

Dans la section 7 , on s'intéresse à la régularité du bord de l'ouvert convexe. C'est là qu'on montre le théorème 1.4 et le corollaire 1.5. Une bonne partie de cette section est dédiée au théorème 1.6, via le théorème 1.7 dont la démonstration présente quelques technicités.

La section 8 construit le contre-exemple de la proposition 1.1 et détaille la proposition 1.10. Enfin, dans la section 9, on montre les théorèmes 1.11 et 1.12 qui lient exposant critique et entropie volumique. Là encore, les démonstrations présentent quelques difficultés techniques.

\section{Géométries de Hilbert}

2A. Distance et volume. Une carte affine $A$ de $\mathbb{P}^{n}$ est le complémentaire d'un hyperplan projectif. Une carte affine possède une structure naturelle d'espace affine. Un ouvert $\Omega$ de $\mathbb{P}^{n}$ différent de $\mathbb{P}^{n}$ est convexe lorsqu'il est inclus dans une carte affine et qu'il est convexe dans cette carte. Un ouvert convexe $\Omega$ de $\mathbb{P}^{n}$ est dit proprement convexe lorsqu'il existe une carte affine contenant son adhérence $\bar{\Omega}$. Autrement dit, un ouvert convexe est proprement convexe lorsqu'il ne contient pas de droite affine. Un ouvert proprement convexe $\Omega$ de $\mathbb{P}^{n}$ est dit strictement convexe lorsque son bord $\partial \Omega$ ne contient pas de segment non trivial.

Hilbert a introduit sur un ouvert proprement convexe $\Omega$ de $\mathbb{P}^{n}$ la distance qui porte aujourd'hui son nom. Pour $x \neq y \in \Omega$, on note $p, q$ les points d'intersection de la droite $(x y)$ et du bord $\partial \Omega$ de $\Omega$, de telle façon que $x$ soit entre $q$ et $y$, et $y$ entre $x$ et $p$ (voir figure 2). On pose

$$
d_{\Omega}(x, y)=\frac{1}{2} \ln ([p: q: x: y])=\frac{1}{2} \ln \frac{|q y| \cdot|p x|}{|q x| \cdot|p y|} \quad \text { et } \quad d_{\Omega}(x, x)=0,
$$

où :

1. la quantité $[p: q: x: y]$ désigne le birapport des points $p, q, x, y$;

2. $|\cdot|$ est une norme euclidienne quelconque sur une carte affine $A$ qui contient l'adhérence $\bar{\Omega}$ de $\Omega$.

Le birapport étant une notion projective, il est clair que $d_{\Omega}$ ne dépend ni du choix de $A$, ni du choix de la norme euclidienne sur $A$. 

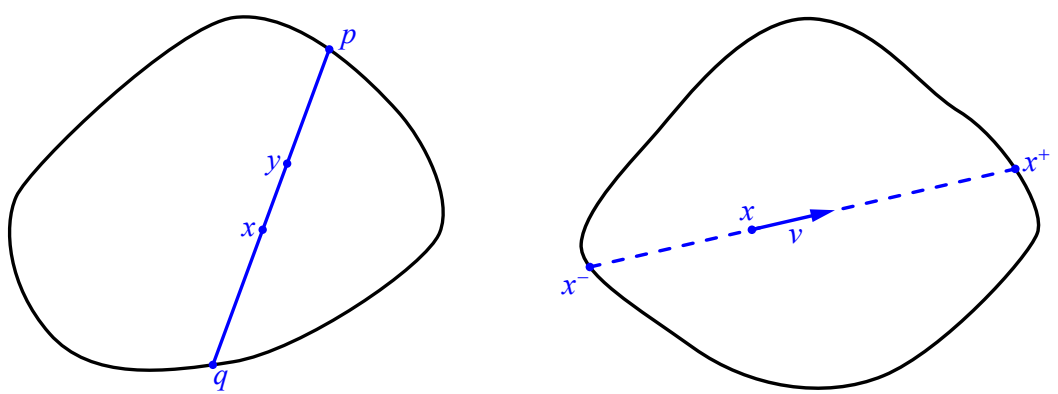

Figure 2. La distance de Hilbert et la norme de Finsler.

Fait 1. Soit $\Omega$ un ouvert proprement convexe de $\mathbb{P}^{n}$.

1. $d_{\Omega}$ est une distance sur $\Omega$.

2. $\left(\Omega, d_{\Omega}\right)$ est un espace métrique complet.

3. La topologie induite par $d_{\Omega}$ coïncide avec celle induite par $\mathbb{P}^{n}$.

4. Le groupe $\operatorname{Aut}(\Omega)$ des transformations projectives de $\mathrm{SL}_{n+1}(\mathbb{R})$ qui préservent $\Omega$ est un sous-groupe fermé de $\mathrm{SL}_{n+1}(\mathbb{R})$ qui agit par isométries sur $\left(\Omega, d_{\Omega}\right)$. Il agit donc proprement sur $\Omega$.

La distance de Hilbert $d_{\Omega}$ est induite par une structure finslérienne sur l'ouvert $\Omega$. On choisit une carte affine $A$ et une métrique euclidienne $|\cdot|$ sur $A$ pour lesquelles $\Omega$ apparaît comme un ouvert convexe borné. On identifie le fibré tangent $T \Omega$ de $\Omega$ à $\Omega \times A$. Soient $x \in \Omega$ et $v \in A$, on note $x^{+}=x^{+}(x, v)$ (resp. $\left.x^{-}\right)$le point d'intersection de la demi-droite définie par $x$ et $v$ (resp. $-v$ ) avec $\partial \Omega$ (voir figure 2). On pose

$$
F(x, v)=\frac{|v|}{2}\left(\frac{1}{\left|x x^{-}\right|}+\frac{1}{\left|x x^{+}\right|}\right),
$$

quantité indépendante du choix de $A$ et de $|\cdot|$, puisqu'on ne considère que des rapports de longueurs.

Fait 2. Soient $\Omega$ un ouvert proprement convexe de $\mathbb{P}^{n}$ et $A$ une carte affine qui contient $\bar{\Omega}$. La distance induite par la métrique finslérienne $F$ est la distance $d_{\Omega}$. Autrement dit on a les formules suivantes:

- $\mathscr{F}(x, v)=\left.\frac{d}{d t}\right|_{t=0} d_{\Omega}(x, x+t v)$, pour $v \in A$.

$-d_{\Omega}(x, y)=\inf \int_{0}^{1} F(\dot{\sigma}(t)) d t$, où l'infimum est pris sur les chemins $\sigma$ de classe $\mathscr{C}^{1}$ tel que $\sigma(0)=x$ et $\sigma(1)=y$.

Il y a plusieurs manières naturelles de construire un volume pour une géométrie de Finsler, la définition riemannienne acceptant plusieurs généralisations. Nous travaillerons avec le volume de Busemann, noté $\mathrm{Vol}_{\Omega}$. 
Pour le construire, on se donne une carte affine $A$ et une métrique euclidienne $|\cdot|$ sur $A$ pour lesquelles $\Omega$ apparaît comme un ouvert convexe borné. On note $B_{T_{x} \Omega}(r)=$ $\left\{v \in T_{x} \Omega \mid F(x, v)<r\right\}$ la boule de rayon $r>0$ de l'espace tangent à $\Omega$ en $x$, Vol la mesure de Lebesgue sur $A$ associée à $|\cdot|$ et $v_{n}=\operatorname{Vol}(\{v \in A|| v \mid<1\})$ le volume de la boule unité euclidienne en dimension $n$.

Pour tout borélien $\mathscr{A} \subset \Omega \subset A$, on pose :

$$
\operatorname{Vol}_{\Omega}(\mathscr{A})=\int_{\mathscr{A}} \frac{v_{n}}{\operatorname{Vol}\left(B_{T_{x} \Omega}(1)\right)} d \operatorname{Vol}(x)
$$

Là encore, la mesure $\mathrm{Vol}_{\Omega}$ est indépendante du choix de $A$ et de $|\cdot|$. En particulier, elle est préservée par le groupe $\operatorname{Aut}(\Omega)$.

La proposition suivante permet de comparer deux géométries de Hilbert entre elles.

Proposition 2.1. Soient $\Omega_{1}$ et $\Omega_{2}$ deux ouverts proprement convexes de $\mathbb{P}^{n}$ tels que $\Omega_{1} \subset \Omega_{2}$.

- Les métriques finslériennes $F_{1}$ et $F_{2}$ de $\Omega_{1}$ et $\Omega_{2}$ vérifient: $F_{2}(w) \leqslant F_{1}(w)$, $w \in T \Omega_{1} \subset T \Omega_{2}$, l'égalité ayant lieu si et seulement si $x_{\Omega_{1}}^{+}(w)=x_{\Omega_{2}}^{+}(w)$ et $x_{\Omega_{1}}^{-}(w)=x_{\Omega_{2}}^{-}(w)$.

- Pour tous $x, y \in \Omega_{1}$, on a $d_{\Omega_{2}}(x, y) \leqslant d_{\Omega_{1}}(x, y)$.

- Les boules métriques et métriques tangentes vérifient, pour tout $x \in \Omega_{1}$ et $r>0$, $B_{\Omega_{1}}(x, r) \subset B_{\Omega_{2}}(x, r)$ et $B_{T_{x} \Omega_{1}}(r) \subset B_{T_{x} \Omega_{2}}(r)$, avec égalité si et seulement si $\Omega_{1}=\Omega_{2}$.

- Pour tout borélien $\mathscr{A}$ de $\Omega_{1}$, on a $\operatorname{Vol}_{\Omega_{2}}(\mathscr{A}) \leqslant \operatorname{Vol}_{\Omega_{1}}(\mathscr{A})$.

2B. Fonctions de Busemann et horosphères. Nous supposons dans ce paragraphe que l'ouvert proprement convexe $\Omega$ de $\mathbb{P}^{n}$ est strictement convexe et à bord $\mathscr{C}^{1}$. Dans ce cadre, il est possible de définir les fonctions de Busemann et les horosphères de la même manière qu'en géométrie hyperbolique, et nous ne donnerons pas de détails.

Pour $\xi \in \partial \Omega$ et $x \in \Omega$, notons $c_{x, \xi}:[0,+\infty) \rightarrow \Omega$ la géodésique issue de $x$ et d'extrémité $\xi$, soit $c_{x, \xi}(0)=x$ et $c_{x, \xi}(+\infty)=\xi$. La fonction de Busemann basée en $\xi \in \partial \Omega b_{\xi}(\cdot, \cdot): \Omega \times \Omega \rightarrow \mathbb{R}$ est définie par :

$$
b_{\xi}(x, y)=\lim _{t \rightarrow+\infty} d_{\Omega}\left(y, c_{x, \xi}(t)\right)-t=\lim _{z \rightarrow \xi} d_{\Omega}(y, z)-d_{\Omega}(x, z), \quad x, y \in \Omega .
$$

L'existence de ces limites est due aux hypothèses de régularité faites sur $\Omega$. Les fonctions de Busemann sont de classe $\mathscr{C}^{1}$.

L'horosphère basée en $\xi \in \partial \Omega$ et passant par $x \in \Omega$ est l'ensemble

$$
\mathscr{H}_{\xi}(x)=\left\{y \in \Omega \mid b_{\xi}(x, y)=0\right\} .
$$




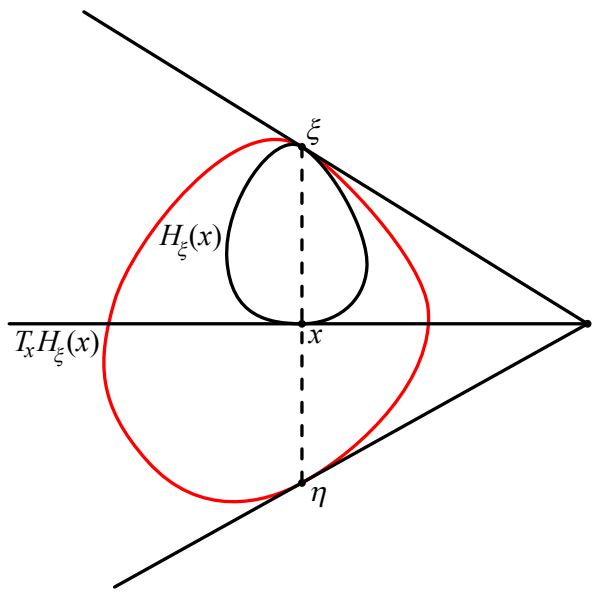

Figure 3. Une horosphère et son espace tangent.

L'horoboule basée en $\xi \in \partial \Omega$ et passant par $x \in \Omega$ est l'ensemble

$$
H_{\xi}(x)=\left\{y \in \Omega \mid b_{\xi}(x, y)<0\right\} .
$$

L'horoboule basée en $\xi \in \partial \Omega$ et passant par $x \in \Omega$ est un ouvert strictement convexe de $\Omega$, dont le bord est l'horosphère correspondante, qui est elle une sous-variété de classe $\mathscr{C}^{1}$ de $\Omega$.

Dans une carte affine $A$ dans laquelle $\Omega$ apparaît comme un ouvert convexe relativement compact, on peut, en identifiant $T \Omega$ avec $\Omega \times A$, construire géométriquement l'espace tangent à $\mathscr{H}_{\xi}(x)$ en $x$ : c'est le sous-espace affine contenant $x$ et l'intersection $T_{\xi} \partial \Omega \cap T_{\eta} \partial \Omega$ des espaces tangents à $\partial \Omega$ en $\xi$ et $\eta=(x \xi) \cap \partial \Omega \backslash\{\xi\}$.

On peut voir que l'horoboule et l'horosphère basées en $\xi \in \partial \Omega$ et passant par $x \in \Omega$ sont les limites des boules et des sphères métriques centrées au point $z \in \Omega$ et passant par $x$ lorsque $z$ tend vers $\xi$.

2C. Dualité. À l'ouvert proprement convexe $\Omega$ de $\mathbb{P}^{n}$ est associé l'ouvert proprement convexe dual $\Omega^{*}$ : on considère un des deux cônes $C \subset \mathbb{R}^{n+1}$ au-dessus de $\Omega$, et son dual

$$
C^{*}=\left\{f \in\left(\mathbb{R}^{n+1}\right)^{*} \mid f(x)>0 \text { pour chaque } x \in C\right\} .
$$

Le convexe $\Omega^{*}$ est par définition la trace de $C^{*}$ dans $\mathbb{P}\left(\left(\mathbb{R}^{n+1}\right)^{*}\right)$.

Le bord de $\partial \Omega^{*}$ est facile à comprendre, car il s'identifie à l'ensemble des hyperplans tangents à $\Omega$. En effet, un hyperplan tangent $T_{x}$ à $\partial \Omega$ en $x$ est la trace d'un hyperplan $H_{x}$ de $\mathbb{R}^{n+1}$. L'ensemble des formes linéaires dont le noyau est $H_{x}$ forme une droite de $\left(\mathbb{R}^{n+1}\right)^{*}$, dont la trace $x^{*}$ dans $\mathbb{P}\left(\left(\mathbb{R}^{n+1}\right)^{*}\right)$ est dans $\partial \Omega^{*}$. Il n'est pas dur de voir qu'on obtient ainsi tout le bord $\partial \Omega^{*}$. 
Cette remarque permet de voir que le dual d'un ouvert strictement convexe a un bord de classe $\mathscr{C}^{1}$, et inversement. En particulier, lorsque $\Omega$ est strictement convexe et que son bord est de classe $\mathscr{C}^{1}$, ce qui est le cas que nous étudierons, on obtient une involution continue $x \mapsto x^{*}$ entre les bords de $\Omega$ et $\Omega^{*}$.

Tout sous-groupe $\Gamma$ de $\operatorname{SL}_{n+1}(\mathbb{R})$ agit par dualité sur $\left(\mathbb{R}^{n+1}\right)^{*}$ et donc sur $\mathbb{P}\left(\left(\mathbb{R}^{n+1}\right)^{*}\right)$, via la formule suivante :

$$
(\gamma \cdot f)(x)=f\left(\gamma^{-1} x\right), \quad \gamma \in \Gamma, x \in \mathbb{R}^{n+1} .
$$

Le convexe dual $\Omega^{*}$ est préservé par un élément $\gamma \in \mathrm{SL}_{n+1}(\mathbb{R})$ si et seulement si $\Omega$ est préservé par $\gamma$. On obtient de cette façon une action de tout sous-groupe $\Gamma$ de $\operatorname{Aut}(\Omega)$ sur le convexe dual $\Omega^{*}$. Le sous-groupe discret de $\operatorname{Aut}\left(\Omega^{*}\right)$ ainsi obtenu sera noté $\Gamma^{*}$. Bien entendu, on a $\left(\Omega^{*}\right)^{*}=\Omega$ et $\left(\Gamma^{*}\right)^{*}=\Gamma$.

\section{Dans tout ce qui suit, sauf mention explicite, $\Omega$ désignera un ouvert propre-} ment convexe, strictement convexe et à bord $\mathscr{C}^{1}$.

2D. Isométries. Les isométries d'une géométrie $\left(\Omega, d_{\Omega}\right)$ avec $\Omega$ strictement convexe à bord $\mathscr{C}^{1}$ ont été classifiées dans [Crampon et Marquis 2012]. Ce sont toutes des transformations projectives qui préservent $\Omega$, et, quitte à considérer leur carré, on les verra donc comme des éléments du groupe linéaire $\mathrm{SL}_{n+1}(\mathbb{R})$, agissant sur $\mathbb{P}^{n}$. Outre les isométries elliptiques qui sont de torsion et qui ne nous intéresseront pas ici, on trouve les isométries hyperboliques et paraboliques.

- Une isométrie hyperbolique $\gamma$ a exactement deux points fixes $x_{\gamma}^{+}, x_{\gamma}^{-} \in \partial \Omega$, l'un répulsif et l'autre attractif. Cela veut dire que la suite $\left(\gamma^{n}\right)_{n \in \mathbb{N}}$ converge uniformément sur les compacts de $\bar{\Omega} \backslash\left\{x_{\gamma}^{-}\right\}$vers $x_{\gamma}^{+}$, et la suite $\left(\gamma^{-n}\right)_{n \in \mathbb{N}}$ converge uniformément sur les compacts de $\bar{\Omega} \backslash\left\{x_{\gamma}^{+}\right\}$vers $x_{\gamma}^{-}$. De plus, les valeurs propres $\lambda_{0}(\gamma)$ et $\lambda_{n}(\gamma)$ associées aux points fixes $x_{\gamma}^{+}$et $x_{\gamma}^{-}$sont positives : c'est une conséquence du fait que le rayon spectral de $\gamma$ est valeur propre de $\gamma$ ([Benoist 2006, lemme 2.3], par exemple); elles sont de multiplicité 1 car, sinon, il y aurait un segment dans le bord de $\Omega$. Finalement, $\gamma$ agit par translation sur le segment ouvert $] x_{\gamma}^{-} x_{\gamma}^{+}[$de $\Omega$, translation de force

$$
\tau(\gamma)=\ln \frac{\lambda_{0}(\gamma)}{\lambda_{n}(\gamma)}
$$

- Une isométrie parabolique $\gamma$ a exactement un point fixe $p \in \partial \Omega$ et préserve toute horosphère basée en $p$. De plus, la famille $\left(\gamma^{n}\right)_{n \in \mathbb{Z}}$ converge uniformément sur les compacts de $\bar{\Omega} \backslash\{p\}$ vers $p$.

On dira qu'un sous-groupe discret $\mathscr{P}$ de $\operatorname{Aut}(\Omega)$, sans torsion, est parabolique si tous ses éléments sont paraboliques. Un tel groupe est nilpotent et ses éléments fixent un même point $p \in \partial \Omega$. On dira que le groupe $\mathscr{P}$ est de rang maximal si son action sur $\partial \Omega \backslash\{p\}$ est cocompacte. 
Si un sous-groupe discret $\Gamma$ de $\operatorname{Aut}(\Omega)$ est donné, on dira qu'un sous-groupe parabolique de $\Gamma$ est maximal s'il n'est contenu dans aucun autre sous-groupe parabolique.

2E. Ensemble limite. Comme en géométrie hyperbolique, on peut définir l'ensemble limite et le domaine de discontinuité d'un sous-groupe discret de $\operatorname{Aut}(\Omega)$ de la façon suivante. On utilise ici de façon essentielle la stricte convexité de $\Omega$.

Définition 2.2. Soit $\Gamma$ un sous-groupe discret de $\operatorname{Aut}(\Omega)$ et $x \in \Omega$. L'ensemble limite $\Lambda_{\Gamma}$ de $\Gamma$ est le sous-ensemble de $\partial \Omega$ suivant :

$$
\Lambda_{\Gamma}=\overline{\Gamma \cdot x} \backslash \Gamma \cdot x
$$

Le domaine de discontinuité $\mathrm{O}_{\Gamma}$ de $\Gamma$ est le complémentaire de l'ensemble limite de $\Gamma$ dans $\partial \Omega$.

L'ensemble limite $\Lambda_{\Gamma}$, s'il n'est pas infini, est vide ou consiste en 1 ou 2 points. On dit que $\Gamma$ est non élémentaire si $\Lambda_{\Gamma}$ est infini. Dans ce dernier cas, l'ensemble limite $\Lambda_{\Gamma}$ est le plus petit fermé $\Gamma$-invariant non vide de $\partial \Omega$. En particulier, $\Lambda_{\Gamma}$ est l'adhérence des points fixes des éléments hyperboliques de $\Gamma$.

Définition 2.3. Soit $\Gamma$ un sous-groupe de $\mathrm{SL}_{n+1}(\mathbb{R})$. On dira que $\Gamma$ est irréductible lorsque les seuls sous-espaces vectoriels de $\mathbb{R}^{n+1}$ invariants par $\Gamma$ sont $\{0\}$ et $\mathbb{R}^{n+1}$. On dira que $\Gamma$ est fortement irréductible si tous ses sous-groupes d'indice fini sont irréductibles, autrement dit, si $\Gamma$ ne préserve pas une union finie de sous-espaces vectoriels non triviaux.

Lemme 2.4. Soit $\Gamma$ un sous-groupe discret de $\operatorname{Aut}(\Omega)$. Les propositions suivantes sont équivalentes:

(i) L'ensemble limite $\Lambda_{\Gamma}$ de $\Gamma$ engendre $\mathbb{P}^{n}$.

(ii) Le groupe $\Gamma$ est irréductible.

(iii) Le groupe $\Gamma$ est fortement irréductible.

Démonstration. L'implication (i) $\Rightarrow$ (ii) vient du fait que $\Lambda_{\Gamma}$ est l'adhérence des points fixes des éléments hyperboliques de $\Gamma$. Pour les implications (ii) $\Rightarrow$ (i) et (iii) $\Rightarrow$ (i), il suffit de voir que l'espace engendré par $\Lambda_{\Gamma}$ est invariant par $\Gamma$.

Montrons pour finir l'implication (i) $\Rightarrow$ (iii). Supposons donc que $\Lambda_{\Gamma}$ engendre $\mathbb{P}^{n}$. Si $G$ est un sous-groupe d'indice fini de $\Gamma$, alors, pour tout élément hyperbolique $h$ de $\Gamma$, il existe un entier $n \geqslant 1$ tel que $h^{n} \in G$. Ainsi, $\Lambda_{G}=\Lambda_{\Gamma}$ et donc $\Lambda_{G}$ engendre $\mathbb{P}^{n}$, ce qui équivaut à l'irréductibilité de $G$. 


\section{Le flot géodésique}

3A. Généralités. Le flot géodésique est le principal objet d'étude de ce travail. Nous le définirons sur le fibré tangent homogène, ou en demi-droites, de $\Omega$, qui est le fibré $\pi: H \Omega \rightarrow \Omega$, avec $H \Omega=(T \Omega \backslash\{0\}) / \mathbb{R}_{+}:$deux points $(x, u)$ et $(y, v)$ de $T \Omega \backslash\{0\}$ sont identifiés si $x=y$ et $u=\lambda v$ pour un certain réel $\lambda>0$.

L'image d'un point $w=(x,[\xi]) \in H \Omega$ par le flot géodésique $\varphi^{t}: H \Omega \rightarrow H \Omega$ est le point $\varphi^{t}(w)=\left(x_{t},\left[\xi_{t}\right]\right)$ obtenu en suivant la géodésique partant de $x$ dans la direction $[\xi]$ pendant le temps $t$. Il est engendré par le champ de vecteurs $X$ sur $H \Omega$, qui a la même régularité que le bord de $\Omega$. Ainsi, $\varphi^{t}$ est au moins de classe $\mathscr{C}^{1}$.

Nous ferons les calculs de façon intelligente en utilisant l'invariance projective. Une carte adaptée à un point $w \in H \Omega$ est une carte affine munie d'une métrique euclidienne telle que :

- la fermeture de $\Omega$ est incluse dans la carte;

- l'intersection des plans tangents à $\partial \Omega$ en $x^{+}$et $x^{-}$sont à l'infini de la carte ; autrement dit, ils y sont parallèles ;

- la droite $\left(x x^{+}\right)$et les plans tangents à $\partial \Omega$ en $x^{+}$et $x^{-}$sont orthogonaux.

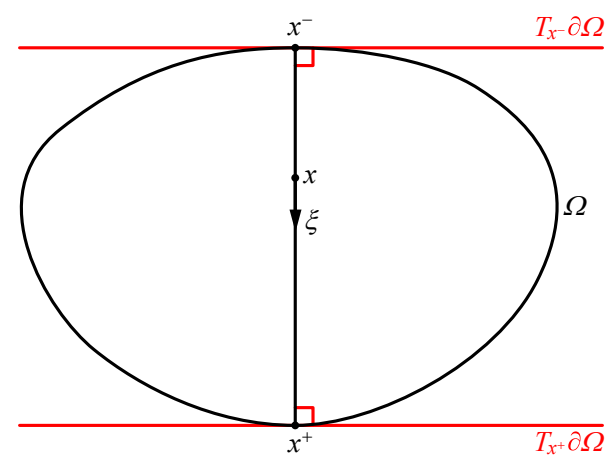

Figure 4. Une carte adaptée en $w$.

3B. Variétés stable et instable. On définit les variétés stable $W^{s}(w)$ et instable $W^{u}(w)$ de $w=(x,[\xi]) \in H \Omega$ par

$$
\begin{aligned}
& W^{s}(w)=\left\{w^{\prime}=\left(y,\left[y x^{+}\right]\right) \mid y \in \mathscr{H}_{x^{+}}(x)\right\}, \\
& W^{u}(w)=\left\{w^{\prime}=\left(y,\left[x^{-} y\right]\right) \mid y \in \mathscr{H}_{x^{-}}(x)\right\} .
\end{aligned}
$$

Il n'est pas difficile de voir que, comme $\Omega$ est strictement convexe à bord $\mathscr{C}^{1}$, on a

$$
\begin{aligned}
& W^{s}(w)=\left\{w^{\prime} \in H \Omega \mid \lim _{t \rightarrow+\infty} d_{\Omega}\left(\pi \varphi^{t}(w), \pi \varphi^{t}\left(w^{\prime}\right)\right)=0\right\}, \\
& W^{u}(w)=\left\{w^{\prime} \in H \Omega \mid \lim _{t \rightarrow-\infty} d_{\Omega}\left(\pi \varphi^{t}(w), \pi \varphi^{t}\left(w^{\prime}\right)\right)=0\right\} .
\end{aligned}
$$




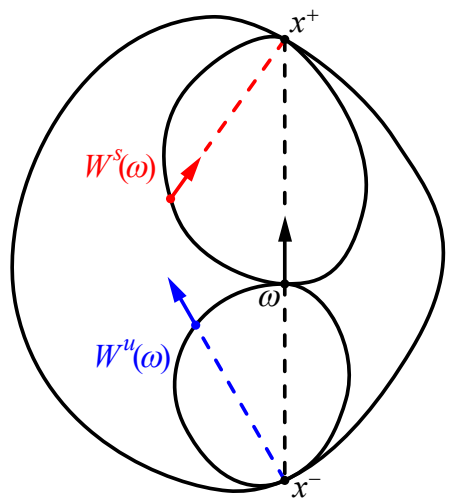

Figure 5. Variétés stable et instable.

Les sous-espaces stable $E^{s}$ et instable $E^{u}$ sont les espaces tangents aux variétés stable et instable. On a clairement que $E^{s} \cap E^{u}=\{0\}$ et donc la décomposition

$$
T H \Omega=\mathbb{R} \cdot X \oplus E^{s} \oplus E^{u},
$$

qu'on appellera décomposition d'Anosov.

On peut définir une norme de Finsler $\|\cdot\|$ sur $H M$ de la façon suivante : pour $Z=a X+Z^{s}+Z^{u} \in \mathbb{R} \cdot X \oplus E^{s} \oplus E^{u}$, on pose

$$
\|Z\|=\left(a^{2}+F\left(d \pi Z^{s}\right)+F\left(d \pi Z^{u}\right)\right)^{1 / 2} .
$$

Cette métrique est précisément celle qui a été introduite dans [Crampon 2014], et qui apparaît naturellement via une décomposition en sous-fibrés horizontaux et verticaux. Nous n'aurons toutefois pas besoin ici de ces notions.

Remarquons qu'en particulier, si $Z$ est un vecteur tangent stable ou instable, c'est-à-dire $Z=Z^{s}$ ou $Z=Z^{u}$, on a $\left\|Z^{s}\right\|=F\left(d \pi Z^{s}\right)$ ou $\left\|Z^{u}\right\|=F\left(d \pi Z^{u}\right)$.

Rappelons les deux lemmes suivants, dont les démonstrations permettront de fixer certaines notations.

Lemme 3.1. Soient $w \in H \Omega$ et $Z \in T_{w} H \Omega$ un vecteur stable (respectivement instable). L'application $t \mapsto\left\|d \varphi^{t}(Z)\right\|$ est une bijection décroissante (respectivement croissante $)$ de $(0,+\infty)$ dans $(0,+\infty)$.

Démonstration. Choisissons une carte adaptée au point $w$. Notons $x=\pi w$ et $x_{t}=\pi \varphi^{t}(w), t \in \mathbb{R}$. Supposons que $Z$ est un vecteur stable, tangent à $H \Omega$ en $w$ et notons $z=d \pi(Z), z_{t}=d \pi d \varphi^{t}(Z), t \in \mathbb{R}$. Rappelons que par définition de la norme, on a $\left\|d \varphi^{t}(Z)\right\|=F\left(z_{t}\right)$. Or,

$$
F\left(z_{t}\right)=\frac{\left|z_{t}\right|}{2}\left(\frac{1}{\left|x_{t} y_{t}^{+}\right|}+\frac{1}{\left|x_{t} y_{t}^{-}\right|}\right)
$$




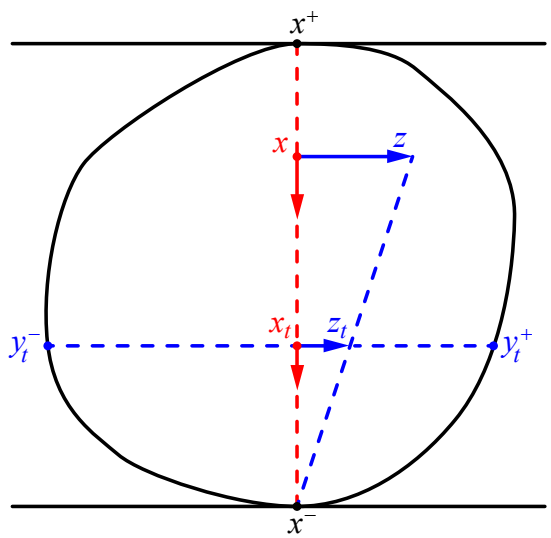

Figure 6. Contraction du flot.

où $y_{t}^{+}$et $y_{t}^{-}$sont les points d'intersection de $x+\mathbb{R} \cdot z$ avec $\partial \Omega$.

Si on considère l'application

$$
h_{t}: y \in \mathscr{H}_{x^{+}}(x) \mapsto y_{t}=\pi \varphi^{t}\left(y,\left[y x^{+}\right]\right)=\left(y x^{+}\right) \cap \mathscr{H}_{x^{+}}\left(x_{t}\right),
$$

on voit que $z_{t}$ est en fait donné par

$$
z_{t}=d h_{t}(z)=\frac{\left|x_{t} x^{+}\right|}{\left|x x^{+}\right|} z
$$

On obtient ainsi

$$
F\left(z_{t}\right)=\frac{|z|}{2\left|x x^{+}\right|}\left(\frac{\left|x_{t} x^{+}\right|}{\left|x_{t} y_{t}^{+}\right|}+\frac{\left|x_{t} x^{+}\right|}{\left|x_{t} y_{t}^{-}\right|}\right) .
$$

Que $t \mapsto\left\|d \varphi^{t}(Z)\right\|$ soit strictement décroissante est alors une conséquence directe de la stricte convexité de $\Omega$. La régularité $\mathscr{C}^{1}$ au point extrémal $\varphi^{+\infty}(w)$ de l'orbite de $w$ entraîne que $\left\|d \varphi^{t}(Z)\right\|$ tend vers 0 en $+\infty$. La stricte convexité à l'autre point extrémal $\varphi^{-\infty}(w)$ de l'orbite de $w$ implique $\lim _{t \rightarrow-\infty}\left\|d \varphi^{t}(Z)\right\|=+\infty$.

Dans le cas où $Z$ est un vecteur instable, on obtient, en gardant les mêmes notations :

$$
F\left(z_{t}\right)=\frac{|z|}{2\left|x x^{-1}\right|}\left(\frac{\left|x_{t} x^{-}\right|}{\left|x_{t} y_{t}^{+}\right|}+\frac{\left|x_{t} x^{-}\right|}{\left|x_{t} y_{t}^{-}\right|}\right) .
$$

On peut donc appliquer le même raisonnement.

Remarquons ici le corollaire suivant, qui dit que la décroissance et la croissance du lemme précédent sont contrôlées.

Corollaire 3.2. Pour tout vecteur $Z \in T H \Omega$, on a

$$
e^{-2|t|}\|Z\| \leqslant\left\|d \varphi^{t}(Z)\right\| \leqslant e^{2|t|}\|Z\| .
$$


Démonstration. Soient $w \in H \Omega$ et $Z^{s} \in E^{s}(w)$ un vecteur stable. Posons $z:=d \pi Z^{s}$. Soit $Z^{u} \in E^{u}(w)$ l'unique vecteur instable tel que $d \pi Z^{u}=z$. On a vu, dans la démonstration du lemme précédent, et avec les mêmes notations que, pour tout $t \in \mathbb{R}$,

et

$$
\left\|d \varphi^{t} Z^{s}\right\|=\frac{|z|}{2\left|x x^{+}\right|}\left(\frac{\left|x_{t} x^{+}\right|}{\left|x_{t} y_{t}^{+}\right|}+\frac{\left|x_{t} x^{+}\right|}{\left|x_{t} y_{t}^{-}\right|}\right),
$$

Ainsi,

$$
\left\|d \varphi^{t} Z^{u}\right\|=\frac{|z|}{2\left|x x^{-}\right|}\left(\frac{\left|x_{t} x^{-}\right|}{\left|x_{t} y_{t}^{+}\right|}+\frac{\left|x_{t} x^{-}\right|}{\left|x_{t} y_{t}^{-}\right|}\right) .
$$

$$
\frac{\left\|d \varphi^{t} Z^{s}\right\|}{\left\|d \varphi^{t} Z^{u}\right\|}=\frac{\left|x_{t} x^{+}\right|}{\left|x_{t} x^{-}\right|} \frac{\left|x x^{+}\right|}{\left|x x^{-}\right|} .
$$

L'égalité $d_{\Omega}\left(x, x_{t}\right)=t$ implique directement que

$$
\frac{\left|x_{t} x^{+}\right|}{\left|x_{t} x^{-}\right|} \frac{\left|x x^{+}\right|}{\left|x x^{-}\right|}=e^{-2 t}
$$

et donc

$$
\frac{\left\|d \varphi^{t} Z^{s}\right\|}{\left\|d \varphi^{t} Z^{u}\right\|}=e^{-2 t} .
$$

Maintenant, le fait que la fonction $t \mapsto\left\|d \varphi^{t} Z^{s}\right\|$ soit décroissante implique que

$$
\limsup _{t \rightarrow+\infty} \frac{1}{t} \ln \left\|d \varphi^{t} Z^{s}\right\| \leqslant 0 ;
$$

de même, comme $t \mapsto\left\|d \varphi^{t} Z^{u}\right\|$ est croissante,

$$
\liminf _{t \rightarrow+\infty} \frac{1}{t} \ln \left\|d \varphi^{t} Z^{u}\right\| \geqslant 0 .
$$

De l'égalité (3-2), on déduit donc que

et

$$
\liminf _{t \rightarrow+\infty} \frac{1}{t} \ln \left\|d \varphi^{t} Z^{s}\right\| \geqslant-2
$$

$$
\limsup _{t \rightarrow+\infty} \frac{1}{t} \ln \left\|d \varphi^{t} Z^{u}\right\| \leqslant 2 .
$$

Ainsi, on a, pour tout $t \geqslant 0$ et $Z^{s} \in E^{s} \backslash\{0\}$,

$$
e^{-2|t|} \leqslant \frac{\left\|d \varphi^{t}\left(Z^{s}\right)\right\|}{\left\|Z^{s}\right\|} \leqslant 1 .
$$

De manière similaire, on obtient pour tout $t \geqslant 0$ et $Z^{u} \in E^{u} \backslash\{0\}$,

$$
1 \leqslant \frac{\left\|d \varphi^{t}\left(Z^{u}\right)\right\|}{\left\|Z^{u}\right\|} \leqslant e^{2|t|} .
$$

On obtient le résultat en décomposant un vecteur $Z$ selon $E^{s} \oplus E^{u} \oplus \mathbb{R} \cdot X$. 
3C. Ensemble non errant. Nous voulons par la suite étudier des propriétés de récurrence du flot géodésique. Pour cela, il nous faut regarder l'ensemble des points qui ne partent pas pour toujours à l'infini.

Étant donnée la variété $M=\Omega / \Gamma$, on notera $H M$ le fibré tangent homogène de $M$, quotient du fibré $H \Omega$ par le groupe $\Gamma$. L'ensemble non errant du flot géodésique de $M$ est l'ensemble fermé NW des points $w \in H M$ dont l'orbite passe une infinité de fois dans tout voisinage ouvert de $w$, dans le passé et dans le futur. Cet ensemble est naturellement relié à l'ensemble limite : c'est la projection sur $H M$ de l'ensemble

$$
\left\{w=(x, \xi) \in H \Omega \mid x^{+}(w), x^{-}(w) \in \Lambda_{\Gamma}\right\} .
$$

En particulier, la projection de NW sur $M$ est incluse dans le cœur convexe de $M$; cela nous permettra d'utiliser le fait 3 ci-dessous lorsque nous seront confrontés à des variétés géométriquement finies.

\section{Variétés géométriquement finies}

4A. Décomposition du cour convexe. Les variétés géométriquement finies sont le contexte de cet article. Des définitions équivalentes de la finitude géométrique ont été données dans [Crampon et Marquis 2012]. Rappelons seulement le résultat suivant, essentiel dans le présent travail (voir [Crampon et Marquis 2012, section 8]) :

Fait 3. Soit $M=\Omega / \Gamma$ une variété géométriquement finie. Le cœur convexe $C(M)$ de $M$ est l'union d'un compact $K$ et d'un nombre fini de pointes

$$
\mathscr{C}_{i}=\left(H_{i} \cap \overline{C\left(\Lambda_{\Gamma}\right)^{\Omega}}\right) / \mathscr{P}_{i}, \quad 1 \leqslant i \leqslant l,
$$

où $H_{i}$ est une horoboule basée en un point $p_{i} \in \partial \Omega$, et $\mathscr{P}_{i}$ est le sous-groupe parabolique maximal de $\Gamma$ fixant $p_{i}$, soit $\mathscr{P}_{i}=\operatorname{Stab}_{\Gamma}\left(p_{i}\right)$.

4B. Groupes paraboliques de rang maximal. Les sous-groupes paraboliques maximaux qui apparaissent ici sont conjugués à des sous-groupes paraboliques d'isométries hyperboliques; c'est un des résultats principaux de [Crampon et Marquis 2012]. Un cas particulier est celui où les sous-groupes paraboliques sont de rang maximal, c'est-à-dire que leur action sur $\partial \Omega \backslash\{p\}$, où $p$ est le point fixe du groupe en question, est cocompacte. Dans ce cas, on a le résultat suivant.

Théorème 4.1 [Crampon et Marquis 2012, section 7]. Soit $\mathscr{P}$ un sous-groupe parabolique de $\operatorname{Aut}(\Omega)$, de rang maximal et de point fixe $p \in \partial \Omega$. Il existe deux ellipsoïdes $\mathscr{P}_{\text {-invariants }} \mathscr{C}^{\mathrm{int}}$ et $\mathscr{E}^{\mathrm{Eext}}$ tels que :

- $\partial^{\mathscr{E}} \mathscr{E}^{\text {int }} \cap \partial_{\mathscr{E}} \mathscr{E}^{\mathrm{ext}}=\partial \mathscr{E}^{\mathrm{int}} \cap \partial \Omega=\partial \mathscr{E}^{\mathrm{ext}} \cap \partial \Omega=\{p\} ;$

$-\mathscr{E}^{\text {int }} \subset \Omega \subset \mathscr{E}^{\text {ext }}$

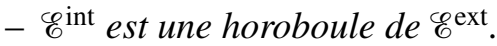


Anticipons un peu. Pour pouvoir dire quelque chose du flot géodésique d'une variété géométriquement finie, il va nous falloir maîtriser ce qui se passe dans les parties qui partent à l'infini, les pointes $\mathscr{C}_{i}$. De façon générale, cela ne sera pas possible, comme le montre le contre-exemple que nous donnons dans la partie 8.1. Toutefois, lorsque les sous-groupes paraboliques sont de rang maximal, les deux ellipsoïdes du théorème précédent nous donnent deux métriques hyperboliques dans chaque pointe qui contrôlent la métrique de Hilbert.

Corollaire 4.2. Soit $M=\Omega / \Gamma$ une variété géométriquement finie. Supposons que les sous-groupes paraboliques maximaux de $\Gamma$ soient tous de rang maximal. Alors, pour toute constante $C>1$, on peut trouver une décomposition

$$
C(M)=K \sqcup \bigsqcup_{1 \leqslant i \leqslant l} \mathscr{C}_{i}
$$

du coeur convexe de $M$, et, sur chaque $\mathscr{C}_{i}$, deux métriques hyperboliques $\mathrm{h}_{i}$ et $\mathrm{h}_{i}^{\prime}$, telles que:

- $F, \mathrm{~h}_{i}$ et $\mathrm{h}_{i}^{\prime}$ ont les mêmes géodésiques, à paramétrisation près;

$-C^{-1} \mathrm{~h}_{i} \leqslant \mathrm{~h}_{i}^{\prime} \leqslant F \leqslant \mathrm{~h}_{i} \leqslant C \mathrm{~h}_{i}^{\prime}$.

Démonstration. Soit $p \in \Lambda_{\Gamma}$ un point parabolique, $\mathscr{P}$ le sous-groupe parabolique maximal de $\Gamma$ fixant $p$. Soient $\mathscr{E}^{\text {int }}$ et $\mathscr{E}^{\text {ext }}$ deux ellipsoïdes donnés par le théorème 4.1. Ils définissent deux métriques hyperboliques $\mathrm{h}$ et $\mathrm{h}^{\prime}$ telles que $\mathrm{h}^{\prime} \leqslant F \leqslant \mathrm{~h}$.

L'adhérence de Zariski $U$ de $\mathscr{P}$ dans $\mathrm{SL}_{n+1}(\mathbb{R})$ est isomorphe à $\mathbb{R}^{n-1}$. Les ensembles $\partial^{\mathscr{E}^{\text {int }}} \backslash\{p\}$ et $\partial^{\mathscr{C} \mathscr{E}}{ }^{\text {ext }} \backslash\{p\}$ sont des orbites de $\mathcal{U}$. Par exemple, dans une

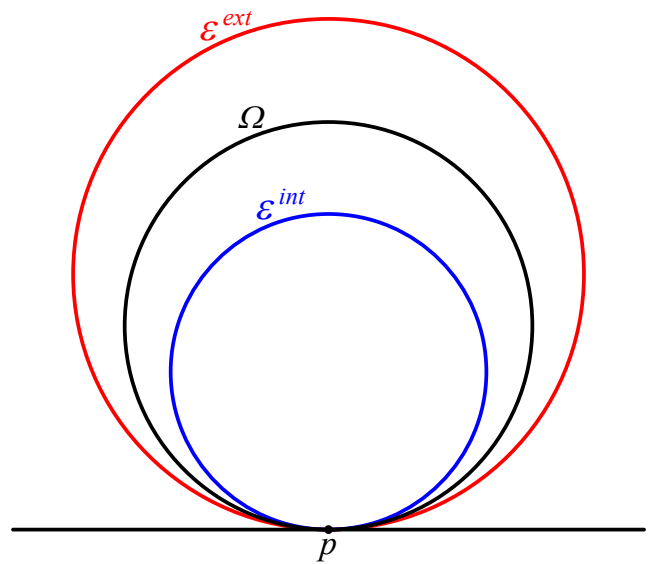

Figure 7. Les ellipsoïdes tangents. 
certaine base de $\mathbb{R}^{n+1}, \partial \mathscr{E}$ ext est d'équation

$$
x_{n} x_{n+1}=x_{1}^{2}+\cdots+x_{n-1}^{2},
$$

et alors $\partial_{\mathscr{E}}^{\text {int }}$ est d'équation

$$
-x_{n}^{2}+2 a x_{n} x_{n+1}=2 a\left(x_{1}^{2}+\cdots+x_{n-1}^{2}\right)
$$

pour un certain $a>0$.

Soient $C>1$ et $x$ un point de $\mathscr{E}^{\text {int }}$. Pour $t \geqslant 0$, on note $x(t)$ le point du segment $[x p]$ tel que $d_{\Omega}(x, x(t))=t$. On considère la fonction

$$
f_{x}: t \mapsto \max _{v \in T_{x(t)} \Omega \backslash\{0\}} \frac{\mathrm{h}(x(t), v)}{\mathrm{h}^{\prime}(x(t), v)} .
$$

Lemme 4.3. Il existe $T=T(C)$ tel que, pour tout $t \geqslant T, 1 \leqslant f_{x}(t) \leqslant C$.

Démonstration. Pour simplifier les calculs, on peut supposer que les équations de $\partial^{\mathscr{E}} \mathscr{E}^{\text {ext }}$ et $\partial^{\mathscr{C}}{ }^{\text {int }}$ sont les précédentes et qu'on travaille dans la carte affine $\left\{x_{n+1}=1\right\}$ avec la structure euclidienne induite par celle de $\mathbb{R}^{n+1}$. On peut aussi supposer que le point $x$ a pour coordonnées $\left(0, \ldots, 0, x_{n}\right)$ pour un certain $x_{n}>0$; le point $p$ est ici l'origine et $T_{p} \partial_{\mathscr{E}}^{\text {ext }}=\left\{t_{n}=0\right\}$. La définition de la métrique de Finsler (formule (2-1)) donne immédiatement que, pour $v \in \mathbb{R} \cdot x p \backslash\{0\}$ ou $v \in\left\{t_{n}=0\right\} \backslash\{0\}$,

$$
\lim _{t \rightarrow+\infty} \frac{\mathrm{h}(x(t), v)}{\mathrm{h}^{\prime}(x(t), v)}=1 .
$$

Il existe donc $T(C)$ tel que pour $t \geqslant T(C)$ on ait $\frac{\mathrm{h}(x(t), v)}{\mathrm{h}^{\prime}(x(t), v)} \leqslant C$ pour $v \in \mathbb{R} \cdot x p \backslash\{0\}$ ou $v \in\left\{t_{n}=0\right\} \backslash\{0\}$.

De plus, pour tout $t \geqslant 0$, les sous-espaces $\mathbb{R} \cdot x p$ et $\left\{t_{n}=0\right\}$ de $T_{x(t)} \Omega$ sont orthogonaux, tant pour $\mathrm{h}(x(t), \cdot)$ que pour $\mathrm{h}^{\prime}(x(t), \cdot)$. En décomposant le vecteur $v \in T_{x(t)} \Omega \backslash\{0\}$ selon $\mathbb{R} \cdot x p$ et $\left\{t_{n}=0\right\}$, on voit que

$$
\frac{\mathrm{h}(x(t), v)}{\mathrm{h}^{\prime}(x(t), v)} \leqslant C
$$

dès que $t \geqslant T(C)$.

Comme les métriques $\mathrm{h}$ et $\mathrm{h}^{\prime}$ sont invariantes par $U$, on a aussi $1 \leqslant f_{u \cdot x}(t) \leqslant C$, pour tout $u \in \mathcal{U}$ et tout $t \geqslant T$. Il existe donc une horoboule $H^{\text {int }}$ de $\mathscr{E}^{\text {int }}$ basée en $p$ telle que, sur $H^{\text {int }}, C^{-1} \mathrm{~h} \leqslant \mathrm{~h}^{\prime} \leqslant F \leqslant \mathrm{~h} \leqslant C \mathrm{~h}^{\prime}$. Comme $\mathscr{P}$ agit de façon cocompacte sur $\partial \Omega \backslash\{p\}, H^{\text {int }}$ contient une horoboule $H$ de $\Omega$ basée en $p$ telle que, sur $H$ :

- $F, \mathrm{~h}$ et $\mathrm{h}^{\prime}$ ont les mêmes géodésiques (les droites), à paramétrisation près ;

$-C^{-1} \mathrm{~h} \leqslant \mathrm{~h}^{\prime} \leqslant F \leqslant \mathrm{~h} \leqslant C \mathrm{~h}^{\prime}$.

On peut maintenant conclure. Considérons un ensemble de représentants $\left\{p_{i}\right\}_{i=1}^{l}$ des points paraboliques de $\Lambda_{\Gamma}$. On note $\mathscr{P}_{i}$ le sous-groupe parabolique maximal de $\Gamma$ 
qui fixe le point $p_{i}$. On peut faire la construction précédente pour chaque point $p_{i}$. On obtient ainsi une horoboule $H_{i}$ de $\Omega$ basée en $p_{i}$ et, sur $H_{i}$, deux métriques hyperboliques $\mathscr{P}_{i}$-invariantes $\mathrm{h}_{i}$ et $\mathrm{h}_{i}^{\prime}$, vérifiant les propriétés précédentes. Elles induisent par projection deux métriques hyperboliques sur la pointe $\mathscr{C}_{i}=H_{i} / \mathscr{P}_{i}$, qui satisfont aux conditions de l'énoncé.

On peut supposer que $H_{i} \subset C\left(\Lambda_{\Gamma}\right), 1 \leqslant i \leqslant l$. D'après le fait 3 , il est aussi possible de prendre les $H_{i}$ telles que l'union

$$
\bigcup_{\substack{\gamma \in \Gamma \\ 1 \leqslant i \leqslant l}} \gamma \cdot H_{i}
$$

soit disjointe. La pointe $\mathscr{C}_{i}=H_{i} / \mathscr{P}_{i}$ s'identifie ainsi à une partie de $C(M)$. L'ensemble $K=C(M) \backslash \bigsqcup_{i} \mathscr{C}_{i}$ est nécessairement compact et cela donne la décomposition annoncée.

4C. Pointes asymptotiquement hyperboliques. Nous allons suivre le chemin indiqué par les groupes paraboliques de rang maximal en nous restreignant à ces variétés géométriquement finies dont nous savons contrôler la métrique de Hilbert dans les pointes :

Définition 4.4. On dira qu'une variété $M=\Omega / \Gamma$ géométriquement finie est $\grave{a}$ pointes asymptotiquement hyperboliques s'il existe une décomposition du cœur convexe $C(M)=K \sqcup \bigsqcup_{1 \leqslant i \leqslant l} \mathscr{C}_{i}$ telle que, sur chaque $\mathscr{C}_{i}$, il existe une métrique hyperbolique $\mathrm{h}_{i}$, ayant les mêmes géodésiques (non paramétrées) que $F$ et qui soit équivalente à $F$, c'est-à-dire que, pour un certain $C_{i} \geqslant 1$,

$$
C_{i}^{-1} \mathrm{~h}_{i} \leqslant F \leqslant C_{i} \mathrm{~h}_{i} .
$$

Si la condition d'être géométriquement fini porte sur le groupe $\Gamma$, celle d'hyperbolicité asymptotique des pointes porte sur $\Omega$. Le lemme 4.6 qui suit donne une condition sur le bord de $\Omega$, inspirée par les observations précédentes, pour que les pointes soient asymptotiquement hyperboliques. Pour l'énoncer, nous faisons quelques rappels :

Définition 4.5. Soient $\varepsilon>0, \beta>1$. On dit qu'une fonction $f: U \subset \mathbb{R}^{n} \rightarrow \mathbb{R}$, définie et de classe $\mathscr{C}^{1}$ sur un ouvert $U$, est :

- de classe $\mathscr{C}^{1+\varepsilon}$ si, pour une certaine constante $C>0$,

$$
\left|f(x)-f(y)-d_{x} f(y-x)\right| \leqslant C|x-y|^{1+\varepsilon}, \quad x, y \in U ;
$$

- $\beta$-convexe si, pour une certaine constante $C>0$,

$$
\left|f(x)-f(y)-d_{x} f(y-x)\right| \geqslant C|x-y|^{\beta}, \quad x, y \in U .
$$


On dit que $f$ est de classe $\mathscr{C}^{1+\varepsilon}$ ou $\beta$-convexe en un point $x \in U$ si on a les inégalités précédentes pour tout $y$ dans un voisinage de $x$.

Lemme 4.6. Soit $M=\Omega / \Gamma$ une variété géométriquement finie. Si le bord $\partial \Omega$ est de classe $\mathscr{C}^{1+1}$ et 2 -convexe en chaque point parabolique de $\Lambda_{\Gamma}$, alors la variété $M$ est à pointes asymptotiquement hyperboliques.

Démonstration. L'hypothèse de régularité de $\partial \Omega$ aux points paraboliques nous permet, pour chaque point parabolique $p$ de $\Lambda_{\Gamma}$ de stabilisateur le groupe $\mathscr{P}=\operatorname{Stab}_{\Gamma}(p)$, de trouver deux ellipsoïdes $\mathscr{E}^{\text {int }}$ et $\mathscr{E}^{\text {ext }}$ tels que :

$-\partial_{\mathscr{E}}^{\mathscr{i n t}} \cap \partial_{\mathscr{E}}^{\mathscr{E} \text { ext }}=\partial^{\mathscr{E}} \mathscr{E}^{\text {int }} \cap \partial \Omega=\partial^{\mathscr{C}} \mathscr{E}^{\text {ext }} \cap \partial \Omega=\{p\} ;$

$-\mathscr{E}^{\text {int }} \subset \Omega \subset \mathscr{E}^{\text {ext }}$.

Comme les sous-groupes paraboliques de $\Gamma$ sont conjugués à des sous-groupes de $\mathrm{SO}_{n, 1}(\mathbb{R})$, on peut choisir ces deux ellipsoïdes de telle façon qu'ils soient $\mathscr{P}$ invariants. Soit $H$ une horoboule de $\Omega$ basée en $p$, d'adhérence incluse dans $\mathscr{E}^{\text {int }}$. Les ellipsoïdes $\mathscr{E}^{\text {int }}$ et $\mathscr{E}^{\text {ext }}$ définissent sur $H$ deux métriques hyperboliques $\mathscr{P}$ invariantes $\mathrm{h}$ et $\mathrm{h}^{\prime}$, qui ont les mêmes géodésiques que $F$, et telles que $\mathrm{h}^{\prime} \leqslant F \leqslant \mathrm{~h}$.

On peut maintenant voir qu'il existe une constante $C \geqslant 1$ telle que $1 / C \leqslant \mathrm{~h} / \mathrm{h}^{\prime} \leqslant C$ sur $H \cap C\left(\Lambda_{\Gamma}\right)$. Comme $H$ est $\mathscr{P}$-invariante, il suffit de le montrer sur un domaine fondamental $D$ de $P$ sur $H \cap C\left(\Lambda_{\Gamma}\right)$. Soit $\mathscr{H}$ l'horosphère au bord de $H$. Comme $M$ est géométriquement finie, l'intersection $\mathscr{D}=\bar{D}^{\Omega} \cap \mathscr{H}$ est compacte. Il suffit donc de voir que pour tout $x \in \mathscr{D}$, la fonction

$$
f_{x}: t \in[0,+\infty) \mapsto \max _{v \in T_{x(t)} \Omega \backslash\{0\}} \frac{\mathrm{h}(x(t), v)}{\mathrm{h}^{\prime}(x(t), v)}
$$

est bornée dans $(0,+\infty)$, où $x(t)$ est le point du segment $[x p]$ tel que $d_{\Omega}(x, x(t))=t$. C'est un petit calcul.

Comme les métriques $\mathrm{h}$ et $\mathrm{h}^{\prime}$ sont $\mathscr{P}$-invariantes, elles donnent deux métriques hyperboliques sur le quotient $H \cap C\left(\Lambda_{\Gamma}\right) / \mathscr{P}$, qui vérifient les conditions voulues. On conclut alors comme dans la démonstration du corollaire 4.2.

On doit pouvoir obtenir la même conclusion que celle du corollaire 4.2 sous l'hypothèse que le bord $\partial \Omega$ est deux fois différentiable en chaque point parabolique de $\Lambda_{\Gamma}$. Toutefois, cette observation plus précise ne nous sera pas utile dans ce texte : nous n'utiliserons que le corollaire 4.2, dans la section 9 .

De façon générale, les sous-groupes paraboliques étant conjugués à des sousgroupes de $\mathrm{SO}_{n, 1}(\mathbb{R})$, on peut se poser la :

Question 4.7. Soit $\Gamma$ un sous-groupe discret de $\operatorname{Aut}(\Omega)$ dont l'action est géométriquement finie. Existe t-il un ouvert convexe $\Omega^{\prime}$ sur lequel $\Gamma$ agit de façon géométriquement finie à pointes asymptotiquement hyperboliques ? 
Beaucoup de résultats dynamiques ne dépendent pas du convexe que l'on considère et le résultat précédent permettrait de se ramener à une situation géométrique et dynamique agréable, qui sera notre propos dans cette article. Par exemple, le spectre des longueurs ne dépend que du groupe $\Gamma$, les longueurs des géodésiques fermées étant données par les valeurs propres des éléments hyperboliques du groupe.

4D. Cas particuliers. Parmi les variétés géométriquement finies, on peut distinguer celles qui ont volume fini, et celles dont le cœur convexe est compact.

Dans [Crampon et Marquis 2012], on a pu voir que les quotients $\Omega / \Gamma$ qui ont volume fini sont précisément les variétés géométriquement finies dont l'ensemble limite est le bord $\partial \Omega$ tout entier. En particulier, si $\Omega / \Gamma$ est une variété de volume fini, les sous-groupes paraboliques maximaux de $\Gamma$ sont de rang maximal. Remarquons que dans tous les cas, une pointe $\mathscr{C}$ d'une variété géométriquement finie a un volume fini (voir [ibid., partie 8]).

Les variétés convexes cocompactes sont celles dont le cœur convexe est compact; autrement dit, le quotient $\Omega / \Gamma$ est géométriquement finie et le groupe $\Gamma$ ne contient pas d'éléments paraboliques.

\section{Hyperbolicité uniforme du flot géodésique}

Rappelons d'abord quelques définitions.

Définition 5.1. Soit $W$ une variété munie d'une métrique de Finsler $\|\cdot\|$ continue. Soient $\varphi^{t}: W \rightarrow W$ un flot de classe $\mathscr{C}^{1}$ engendré par le champ de vecteurs $X$ sur $W$, et $V$ une partie $\varphi^{t}$-invariante de $W$. On dit que le flot $\varphi^{t}$ est uniformément hyperbolique sur $V$ s'il existe une décomposition $\varphi^{t}$-invariante

$$
T W=\mathbb{R} \cdot X \oplus E^{s} \oplus E^{u}
$$

du fibré tangent à $W$ en tout point de $V$, et des constantes $a, C>0$ pour lesquelles $\left\|d \varphi^{t} Z^{s}\right\| \leqslant C e^{-a t}\left\|Z^{s}\right\| \quad$ et $\quad\left\|d \varphi^{-t} Z^{u}\right\| \leqslant C e^{-a t}\left\|Z^{u}\right\|, \quad Z^{s} \in E^{s}, Z^{u} \in E^{u}, t \geqslant 0$.

Dans le cas où $W$ est une variété compacte et $V=W$, on parle plus souvent de flot d'Anosov. Les distributions $E^{s}$ et $E^{u}$ s'appellent les distributions stables et instables du flot. Le but de cette partie est de montrer une telle propriété d'hyperbolicité pour notre flot géodésique, restreint à son ensemble non errant.

Dans le cas où la variété $M$ est compacte, l'ensemble non errant est $H M$ tout entier, et Yves Benoist a déjà prouvé que le flot géodésique était d'Anosov. Si la variété $M$ est convexe-cocompacte, c'est-à-dire que son cœur convexe est compact, l'ensemble non errant est lui-même compact, et une démonstration similaire fonctionnerait pour prouver l'uniforme hyperbolicité sur l'ensemble non errant. 
Nous étendons ce résultat au cas d'une variété géométriquement finie à pointes asymptotiquement hyperboliques :

Théorème 5.2. Soit $M=\Omega / \Gamma$ une variété géométriquement finie à pointes asymptotiquement hyperboliques. Le flot géodésique est uniformément hyperbolique sur l'ensemble non errant, de décomposition

$$
T H M=\mathbb{R} \cdot X \oplus E^{s} \oplus E^{u} .
$$

Nous montrerons le théorème en plusieurs temps. Fixons une fois pour toutes une décomposition du cœur convexe $C(M)$ de $M$ en une partie compacte $K$ et une union finie de pointes $\mathscr{C}_{i}, 1 \leqslant i \leqslant l$, chacun d'entre eux portant une métrique hyperbolique $\mathrm{h}_{i}$ telle que :

- $F$ et $\mathrm{h}_{i}$ ont les mêmes géodésiques, à paramétrisation près ;

- $C^{-1} \mathrm{~h}_{i} \leqslant F \leqslant C \mathrm{~h}_{i}$, pour une certaine constante $C \geqslant 1$.

Pour la partie compacte, on se servira du lemme suivant :

Lemme 5.3. Soient $V$ une partie compacte de $H M$ et $T>0$. Il existe un réel $0<b(V, T)<1$ tel que, si $\varphi^{t}(w) \in V$ pour $0 \leqslant t \leqslant T$, alors, pour tout $Z \in E^{s}(w)$,

$$
\left\|d \varphi^{T}(Z)\right\| \leqslant b(V, T)\|Z\| .
$$

Démonstration. C'est une simple conséquence du lemme 3.1. Notons $V_{T}$ l'ensemble des $w \in V$ tels que $\varphi^{t}(w) \in V$ pour $0 \leqslant t \leqslant T$ et $E_{1}=\left\{Z \in E^{s}(w) \mid w \in V_{T},\|Z\|=1\right\}$. Les ensembles $V_{T}$ et $E_{1}$ sont compacts. La fonction $Z \in E_{1} \mapsto\left\|d \varphi^{T}(Z)\right\|$ est continue et atteint donc son maximum pour un certain vecteur $Z_{M}$. Le lemme 3.1 nous dit que $\left\|d \varphi^{T}\left(Z_{M}\right)\right\|<1$, d'où le résultat.

Pour les pointes, c'est un peu plus délicat. Choisissons une des pointes $\mathscr{C}_{i}$, et oublions les indices : on note $\mathscr{C}$ la pointe et h la métrique hyperbolique $\operatorname{sur} \mathscr{C}$.

Lemme 5.4. Pour tout $0<a<1$, on peut trouver un temps $T_{a}=T_{a}(C)>0$ tel que, pour tout $w \in H^{\mathscr{C}}$ tel que $\varphi^{t}(w) \in H^{\mathscr{C}}$ pour $0 \leqslant t \leqslant T_{a}$ et $Z \in E^{s}(w)$, on a

$$
\left\|d \varphi^{T_{a}} Z\right\| \leqslant a\|Z\| \text {. }
$$

Avant de montrer ce dernier lemme, voyons d'abord comment en déduire le théorème :

Démonstration du théorème 5.2. Rappelons la décomposition du cœur convexe en

$$
C(M)=K \sqcup \bigsqcup_{i} \mathscr{C}_{i}
$$

Choisissons un réel $0<a<1$ et un temps $T_{a}>0$ comme dans le lemme 5.4, et posons

$$
K_{a}=\bigcup_{-T_{a} \leqslant t \leqslant T_{a}} \varphi^{t}\left(\left.H M\right|_{K}\right) .
$$


Pour tout point $w$ de l'ensemble non errant NW, le morceau d'orbite $\left\{\varphi^{t}(w)\right\}_{0 \leqslant t \leqslant T_{a}}$ est inclus soit dans $K_{a}$, soit dans un des $H^{C} \mathscr{C}_{i}$. Les deux lemmes précédents impliquent alors que, pour tout $\left.Z \in E^{s}\right|_{\mathrm{NW}}$, on a

$$
\left\|d \varphi^{T_{a}} Z\right\| \leqslant A\|Z\|,
$$

avec $A=\max \left(a, b\left(K_{a}, T_{a}\right)\right)<1$. Ainsi, pour tout $t \geqslant 0$, en posant $N=\left[t / T_{a}\right]$, on a

$$
\left\|d \varphi^{t}(Z)\right\| \leqslant A^{N}\left\|d \varphi^{t-N T_{a}}(Z)\right\| \leqslant \frac{\left\|d \varphi^{t-N T_{a}}(Z)\right\|}{e^{\frac{t-N T_{a}}{T_{a}} \ln A}} e^{\frac{t}{T_{a}} \ln A} \leqslant A^{-1} e^{-\frac{\ln A^{-1}}{T_{a}} t}\|Z\| .
$$

Cela prouve la décroissance uniformément hyperbolique sur la distribution stable. On fait de même pour la distribution instable en considérant $\varphi^{-t}$.

Le reste de cette partie est consacrée à la démonstration du lemme 5.4. Bien entendu, l'idée est de comparer les flots géodésiques des métriques $F$ et h sur $H^{\mathscr{C}}$, qui satisfont $C^{-1} \mathrm{~h} \leqslant F \leqslant C \mathrm{~h}$ pour une certaine constante $C>1$. Comme $F$ et $\mathrm{h}$ ont les mêmes géodésiques à paramétrisation près, le flot $\varphi^{t}$ est en effet une renormalisation du flot $\varphi_{\mathrm{h}}^{t}$ de la métrique $\mathrm{h}:$ on a

$$
\varphi^{t}(w)=\varphi_{\mathrm{h}}^{\alpha(w, t)}(w)
$$

pour un certain $\alpha(w, t) \in \mathbb{R}$. Bien sûr, cette expression ne fait sens que si $\varphi^{s}(w)$ est dans $H^{\mathscr{C}}$ pour tout $0 \leqslant s \leqslant t$. La fonction $\alpha$ est donc définie sur l'ensemble

$$
W=\left\{(w, t) \mid \varphi^{s}(w) \in H^{\mathscr{C}}, 0 \leqslant s \leqslant t\right\} \subset H^{\mathscr{C}} \times \mathbb{R} .
$$

Soit $g$ la fonction définie sur $H^{\mathscr{C}}$ par $F=g^{-1}$ h. C'est une fonction de classe $\mathscr{C}^{1}$, qui prend ses valeurs dans l'intervalle $\left[C^{-1}, C\right]$. Si $X_{\mathrm{h}}$ est le générateur du flot géodésique de $\mathrm{h}$, alors on a $X=g X_{\mathrm{h}}$. On retrouve la fonction $\alpha$ en intégrant $g$ :

$$
\alpha(w, t)=\int_{0}^{t} g\left(\varphi^{s}(w)\right) d s
$$

la fonction $\alpha$ est donc de classe $\mathscr{C}^{1}$ et satisfait

$$
C^{-1} t \leqslant \alpha(w, t) \leqslant C t, \quad t \geqslant 0 .
$$

L'espace tangent à $H^{\mathscr{C}}$ se décompose de deux façons, selon que l'on considère le flot de $F$ ou de h :

$$
T H \mathscr{C}=E^{s} \oplus E^{u} \oplus \mathbb{R} \cdot X=E_{\mathrm{h}}^{s} \oplus E_{\mathrm{h}}^{u} \oplus \mathbb{R} \cdot X .
$$

Sur $H \mathscr{C}$, on dispose des métriques $\|\cdot\|$ et $\|\cdot\|_{\mathrm{h}}$ associées respectivement à $F$ et h et définies par la formule (3-1) via les décompositions précédentes. La métrique $\|\cdot\|_{\mathrm{h}}$ est bien entendu une métrique riemannienne, qui n'est rien d'autre que la métrique 


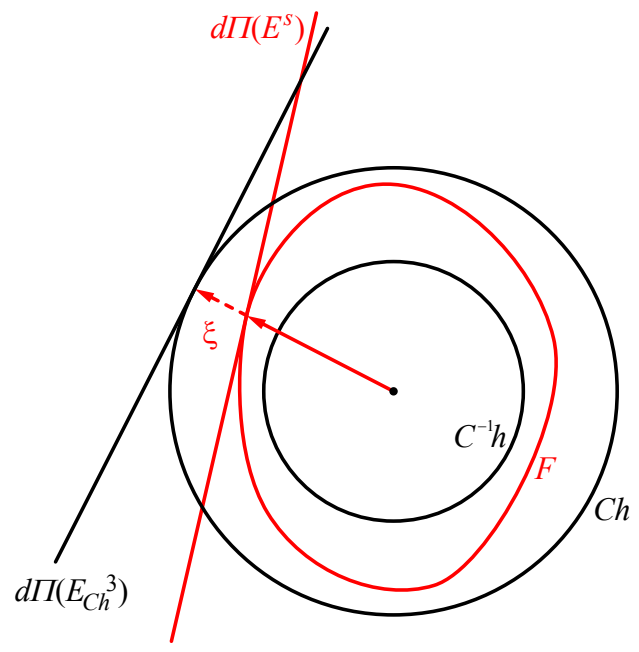

Figure 8. La boule unité de $F$ est coincée entre celles de $C^{-1} \mathrm{~h}$ et $C \mathrm{~h}$.

de Sasaki, et pour laquelle la décomposition $T H \mathscr{C}=E_{\mathrm{h}}^{s} \oplus E_{\mathrm{h}}^{u} \oplus \mathbb{R} \cdot X$ est orthogonale. Rappelons que, si $Z_{\mathrm{h}}^{s} \in E_{\mathrm{h}}^{s}$, alors

$$
\left\|d \varphi_{\mathrm{h}}^{t}\left(Z_{\mathrm{h}}^{s}\right)\right\|=e^{-t}\left\|Z_{\mathrm{h}}^{s}\right\|, \quad t \in \mathbb{R}
$$

sous réserve, bien sûr, que $Z$ soit tangent à $H^{\mathscr{C}}$ en un point $w$ tel que $\varphi_{\mathrm{h}}^{s}(w) \in H^{\mathscr{C}}$ pour $0 \leqslant s \leqslant t$.

Le lemme essentiel est le suivant :

Lemme 5.5. La distribution $E^{s}$ est, sur $H \mathscr{C}$, incluse dans un cône d'angle $\theta$ pour $\|\cdot\|_{\mathrm{h}}$ autour de la distribution $E_{\mathrm{h}}^{s}$, avec $0 \leqslant \theta=\theta(C)<\pi / 2$.

Démonstration. Il revient au même de montrer qu'il existe $\theta$ tel que, pour tout $w=(x,[\xi])$ dans $H^{\mathscr{C}}$, la projection $d \pi\left(E^{s}(w)\right)$ est dans un cône d'angle $\theta$ pour $\mathrm{h}$ autour de $d \pi\left(E_{\mathrm{h}}^{s}(w)\right)$.

De la démonstration de la proposition 3.6 de [Crampon 2009], on peut tirer que la projection $d \pi\left(E^{s}(w)\right)$ coïncide avec l'espace tangent en $\xi \in T_{x} \mathscr{b}$ à la boule unité tangente de la norme $F(x, \cdot)$; où $\xi$ est le vecteur de norme 1 de $[\xi]$. La même chose est bien sûr valable pour $d \pi\left(E_{\mathrm{h}}^{s}(w)\right)$ et la métrique $\mathrm{h}$.

Or, la boule unité tangente de $F$ est coincée entre les boules de rayon $C^{-1}$ et $C$ de h. Cet encadrement et le fait précédent impliquent l'existence de $\theta$.

Tout vecteur $Z^{s} \in E^{s}$ se décompose en

$$
Z^{s}=Z_{\mathrm{h}}^{s}+Z_{\mathrm{h}}^{u}+Z_{\mathrm{h}}^{X} \in E_{\mathrm{h}}^{s} \oplus E_{\mathrm{h}}^{u} \oplus \mathbb{R} \cdot X_{\mathrm{h}} .
$$


On déduit du lemme précédent que l'angle entre $Z^{s}$ et sa projection $Z_{\mathrm{h}}^{s}$ sur $E_{\mathrm{h}}^{s}$ est toujours inférieur à $\theta$; de même en ce qui concerne l'angle, pour h, entre $d \pi Z^{s}$ et $d \pi Z_{\mathrm{h}}^{s}$. D'où :

Corollaire 5.6. Pour tout vecteur $Z^{s} \in E^{s}$, on a

$$
\frac{1}{C}\left\|Z_{\mathrm{h}}^{s}\right\|_{\mathrm{h}} \leqslant\left\|Z^{s}\right\| \leqslant \frac{C}{\cos \theta}\left\|Z_{\mathrm{h}}^{s}\right\|_{\mathrm{h}} .
$$

Démonstration. On a

et

$$
\left\|Z^{s}\right\|=F\left(d \pi Z^{s}\right) \leqslant C h\left(d \pi Z^{s}\right) \leqslant \frac{C}{\cos \theta} \mathrm{h}\left(d \pi Z_{\mathrm{h}}^{s}\right)=\frac{C}{\cos \theta}\left\|Z_{\mathrm{h}}^{s}\right\|_{\mathrm{h}},
$$

$$
\left\|Z_{\mathrm{h}}^{s}\right\|_{\mathrm{h}}=\mathrm{h}\left(d \pi Z_{\mathrm{h}}^{s}\right) \leqslant \mathrm{h}\left(d \pi Z^{s}\right) \leqslant C F\left(d \pi Z^{s}\right)=C\left\|Z^{s}\right\| .
$$

Démonstration du lemme 5.4. Fixons $(w, t) \in W$ et un vecteur stable $Z^{s} \in E^{s}(w)$, qui se décompose en

$$
Z^{s}=Z_{\mathrm{h}}^{s}+Z_{\mathrm{h}}^{u}+Z_{\mathrm{h}}^{X} \in E_{\mathrm{h}}^{s} \oplus E_{\mathrm{h}}^{u} \oplus \mathbb{R} \cdot X_{\mathrm{h}} .
$$

On a donc

$$
d \varphi^{t}\left(Z^{s}\right)=d \varphi^{t}\left(Z_{\mathrm{h}}^{s}\right)+d \varphi^{t}\left(Z_{\mathrm{h}}^{u}\right)+d \varphi^{t}\left(Z_{\mathrm{h}}^{X}\right) .
$$

D'autre part, considérons les fonctions $\varphi$ et $\varphi_{\mathrm{h}}$ définies par

$$
\varphi(w, t)=\varphi^{t}(w), \quad \varphi_{\mathrm{h}}(w, t)=\varphi_{\mathrm{h}}^{t}(w) ;
$$

les fonctions $\varphi$ et $\varphi_{\mathrm{h}}$ sont définies, respectivement, sur $W$ et sur l'ensemble

$$
\left\{(w, t) \mid \varphi_{\mathrm{h}}^{s}(w) \in H^{\mathscr{C}}, 0 \leqslant s \leqslant t\right\} .
$$

On a ainsi $\varphi(w, t)=\varphi_{\mathrm{h}}(w, \alpha(w, t))$, d'où

$$
d \varphi^{t}\left(Z^{s}\right)=\frac{\partial \varphi}{\partial w}(w, t)\left(Z^{s}\right)=\frac{\partial \varphi_{\mathrm{h}}}{\partial t} \frac{\partial \alpha}{\partial w}(w, t)\left(Z^{s}\right)+d \varphi_{\mathrm{h}}^{\alpha(w, t)}\left(Z^{s}\right) .
$$

L'application $\frac{\partial \varphi_{\mathrm{h}}}{\partial t}$ a son image dans $\mathbb{R} \cdot X$; le premier terme

$$
\frac{\partial \varphi_{\mathrm{h}}}{\partial t} \frac{\partial \alpha}{\partial w}(w, t)\left(Z^{s}\right)
$$

de la dernière expression est donc un vecteur de $\mathbb{R} \cdot X$. Comme $d \varphi_{\mathrm{h}}^{t}$ préserve la décomposition $T H \mathscr{C}=E_{\mathrm{h}}^{s} \oplus E_{\mathrm{h}}^{u} \oplus \mathbb{R} \cdot X_{\mathrm{h}}$, on déduit de (5-1) et (5-2) que

$$
\left(d \varphi^{t}\left(Z^{s}\right)\right)_{\mathrm{h}}^{s}=d \varphi_{\mathrm{h}}^{\alpha(w, t)}\left(Z_{\mathrm{h}}^{s}\right) .
$$

On a alors, d'après le corollaire 5.6,

$$
\left\|d \varphi^{t} Z^{s}\right\| \leqslant \frac{C}{\cos \theta}\left\|d \varphi_{\mathrm{h}}^{\alpha(w, t)}\left(Z_{\mathrm{h}}^{s}\right)\right\|_{\mathrm{h}}=\frac{C}{\cos \theta} e^{-\alpha(w, t)}\left\|Z_{\mathrm{h}}^{s}\right\|_{\mathrm{h}} \leqslant \frac{C^{2}}{\cos \theta} e^{-t}\left\|Z^{s}\right\| .
$$


Mais on peut écrire

$$
\frac{C^{2}}{\cos \theta} e^{-t}=e^{-t\left(1-\frac{1}{t} \ln \frac{C^{2}}{\cos \theta}\right)}
$$

Aussi, en prenant $T_{a}=\ln \frac{C^{2}}{a \cos \theta}$, on obtient $\left\|d \varphi^{T_{a}} Z^{s}\right\| \leqslant a\left\|Z^{s}\right\|$.

Remarque 5.7. Dans le lemme 5.5, on pourrait voir que plus $C$ est proche 1 , plus $\theta(C)$ peut être pris proche de 0 . Or, dans la pointe $\mathscr{C}$ choisi pour la constante $C$, on a

$$
\left\|d \varphi^{t} Z^{s}\right\| \leqslant \frac{C^{2}}{\cos \theta(C)} e^{-t}\left\|Z^{s}\right\|,
$$

pour tout vecteur stable $Z^{s}$ tangent en un point $w \in H^{\mathscr{C}}$ tel que $\varphi^{s}(w) \in H^{\mathscr{C}}$, $0 \leqslant s \leqslant t$. En particulier, sous l'hypothèse de rang maximal des sous-groupes paraboliques maximaux, on peut, d'après le corollaire 4.2 , choisir la pointe de telle façon que la constante $C^{2} / \cos \theta(C)$ soit aussi proche de 1 qu'on le souhaite.

\section{Propriétés de récurrence}

6A. Transitivité et mélange topologique. Le but de cette partie est d'étudier les propriétés de récurrence du flot géodésique d'une variété quotient $M=\Omega / \Gamma$ quelconque. Rappelons qu'un flot $\varphi^{t}$ sur un espace topologique $X$ est dit :

- topologiquement transitif s'il existe une orbite dense ou, de façon équivalente, si pour tous ouverts $U$ et $V$ de $X$, il existe $T \in \mathbb{R}$ tel que $\varphi^{T}(U) \cap V \neq \varnothing$;

- topologiquement mélangeant si pour tous ouverts $U$ et $V$ de $X$, il existe $T \in \mathbb{R}$ tel que, pour tout $t \geqslant T, \varphi^{t}(U) \cap V \neq \varnothing$.

Le résultat principal est le suivant.

Proposition 6.1. Soit $M=\Omega / \Gamma$, avec $\Gamma$ non élémentaire. Le flot géodésique est topologiquement mélangeant sur son ensemble non errant.

Une orbite périodique du flot géodésique sur $H M$ se projette sur une géodésique fermée de $M$, parcourue dans un sens ou dans l'autre. Or, les géodésiques fermées orientées sont en bijection avec les classes de conjugaison d'éléments hyperboliques de $\Gamma$. La géodésique fermée orientée définie par un tel $\gamma \in \Gamma$ est précisément la projection sur $M$ de l'axe orienté $\left(x_{\gamma}^{-} x_{\gamma}^{+}\right)$.

À un élément $\gamma \in \Gamma$ correspond ainsi une unique orbite périodique. Bien sûr, l'orbite périodique associée à $\gamma^{-1}$ se projette sur la même géodésique fermée que celle associée à $\gamma$, mais l'orientation est inversée. Le lemme suivant est immédiat :

Lemme 6.2. Soient $g$ et h deux éléments hyperboliques de $\operatorname{Aut}(\Omega)$ tel que l'intersection des groupes engendrés par $g$ et h est triviale. Posons $k_{n}=g^{n} h^{n}$, alors

$$
\lim _{n \rightarrow+\infty} x_{k_{n}}^{+}=x_{g}^{+} \quad \text { et } \quad \lim _{n \rightarrow+\infty} x_{k_{n}}^{-}=x_{h}^{-} .
$$


Corollaire 6.3. Soit $\Gamma$ un sous-groupe de $\operatorname{Aut}(\Omega)$. L'ensemble $\left\{\left(x_{g}^{+}, x_{g}^{-}\right) \mid g \in \Gamma\right\}$ est dense dans $\Lambda_{\Gamma} \times \Lambda_{\Gamma}$. En particulier, les orbites périodiques de HM sont denses dans NW.

Démonstration. Il suffit de se rappeler que l'action de $\Gamma$ sur $\Lambda_{\Gamma}$ est minimale, puisque $\Lambda_{\Gamma}$ est le plus petit fermé $\Gamma$-invariant de $\partial \Omega$; en particulier, l'ensemble des $x_{g}^{+}$pour $g \in \Gamma$ est dense dans $\Lambda_{\Gamma}$. Fixons une métrique riemannienne quelconque sur $\partial \Omega$. Si on prend un couple $(x, y)$ dans $\Lambda_{\Gamma} \times \Lambda_{\Gamma}$, il existe, pour tout $\varepsilon>0$, des éléments $g$ et $h$ hyperboliques de $\Gamma$ tels que $x_{g}^{+}$et $x_{h}^{-}$sont $\varepsilon$-proches de, respectivement, $x$ et $y$. Le lemme précédent affirme alors que pour $n$ assez grand, si $k_{n}=g^{n} h^{n} \in \Gamma$, les points $x_{k_{n}}^{+}$et $x_{k_{n}}^{-}$sont $2 \varepsilon$-proches de, respectivement, $x$ et $y$.

Démonstration de la proposition 6.1. Prenons $U$ et $V$ deux ouverts de NW. Les orbites périodiques étant denses dans NW, il existe une orbite périodique passant dans $U$, et une autre, distincte de la première, passant dans $V$. Considérons des relevés $(x y)$ et $\left(x^{\prime} y^{\prime}\right)$ dans $H \Omega$ de ces orbites. Ce sont les axes d'éléments $\gamma$ et $\gamma^{\prime}$ distincts de $\Gamma$. Le projeté de l'orbite $\left(x y^{\prime}\right)$ sur $H M$ est alors une orbite qui rencontre à la fois $U$ et $V$. Ainsi, il existe $t \geqslant 0$ tel que $\varphi^{t}(U) \cap V \neq \varnothing$ et le flot est topologiquement transitif.

Comme le flot est topologiquement transitif, le mélange topologique est équivalent au fait que le spectre des longueurs des orbites périodiques engendre un sous-groupe dense de $\mathbb{R}$ (exercice 18.3.4 du livre [Katok et Hasselblatt 1995]). Or, la longueur de l'orbite périodique définie par l'élément hyperbolique $\gamma \in \Gamma$ est exactement $\frac{1}{2} \ln \left(\lambda_{0}(\gamma) / \lambda_{n}(\gamma)\right.$ ), où $\lambda_{0}(\gamma)$ et $\lambda_{n}(\gamma)$ sont le module de, respectivement, sa plus grande et plus petite valeur propre. Si le groupe engendré par les longueurs n'était pas dense dans $\mathbb{R}$, il existerait $l>0$ tel que pour tout $\gamma \in \Gamma$, il existe $k_{\gamma} \in \mathbb{N}$ tel que

$$
\frac{1}{2} \ln \frac{\lambda_{0}(\gamma)}{\lambda_{n}(\gamma)}=k_{\gamma} l
$$

Quitte à se restreindre au sous-espace projectif engendré par $\Lambda_{\Gamma}$, on peut supposer que l'action de $\Gamma$ est irréductible. D'après le lemme 2.4, elle est même fortement irréductible. La proposition 6.5 nous dit que l'adhérence de Zariski $G$ de $\Gamma$ est alors un groupe semi-simple. Le théorème 6.4 qui suit implique que la relation (6-1) ne peut être vérifiée pour tout $\gamma \in \Gamma$.

Le théorème permettant de conclure la démonstration est dû à Yves Benoist [2000b]. Rappelons-en ici un énoncé dans notre contexte particulier.

Soit $G$ un sous-groupe de Lie semi-simple de $\mathrm{SL}_{n+1}(\mathbb{R})$. À tout élément $g$ de $G$, on associe le vecteur $\ln (g)=\left(\ln \lambda_{0}(g), \ldots, \ln \lambda_{n}(g)\right) \in \mathbb{R}^{n+1}$, où

$$
\lambda_{0}(g) \geqslant \lambda_{1}(g) \geqslant \cdots \geqslant \lambda_{n}(g)
$$


désignent les modules des valeurs propres de $g$. Pour un sous-groupe $\Gamma$ de $G$, on note $\ln \Gamma$ l'ensemble des $\ln \gamma$ pour $\gamma \in \Gamma$. Le résultat est le suivant :

Théorème 6.4 [ibid.]. Soient $G$ un sous-groupe de Lie semi-simple de $\mathrm{SL}_{n+1}(\mathbb{R})$ et $\Gamma$ un sous-groupe de G. Si $\Gamma$ est Zariski-dense dans $G$, alors le sous-groupe engendré par $\ln \Gamma$ est dense dans le sous-espace vectoriel de $\mathbb{R}^{n+1}$ engendré par $\ln G$.

L'autre résultat que l'on a utilisé était la proposition suivante, due à Yves Benoist. Pour faciliter la lecture de ce texte, nous en donnons une démonstration sous l'hypothèse $\Omega$ strictement convexe, qui n'est toutefois pas nécessaire.

Proposition 6.5 [Benoist 2000a, remarque suivant le corollaire 3.2]. Soit $\Gamma$ un sous-groupe irréductible de $\mathrm{SL}_{n+1}(\mathbb{R})$ qui préserve un ouvert $\Omega \subset \mathbb{P}^{n}$ proprement convexe et strictement convexe. La composante connexe $G$ de l'adhérence de Zariski de $\Gamma$ est un groupe de Lie semi-simple.

Démonstration. D'après le lemme 2.4, $\Gamma$ est fortement irréductible. Quitte à considérer un sous-groupe d'indice fini, on peut donc supposer que $\Gamma$ est Zariski-connexe et ainsi que $G$ est d'indice fini dans l'adhérence de Zariski de $\Gamma$. Le groupe $G$ est Zariski-fermé à indice fini près et agit de façon irréductible sur $\mathbb{R}^{n+1}$. Il est donc réductif. ${ }^{1}$

En effet, soit $N$ un sous-groupe unipotent et distingué de $G$. Considérons le sous-espace vectoriel $E$ de $\mathbb{R}^{n+1}$ des points fixes de $N$. Comme $N$ est distingué dans $G$, l'espace vectoriel $E$ est préservé par $G$. Comme l'action de $G$ sur $\mathbb{R}^{n+1}$ est irréductible, on en déduit que $E$ est trivial. Or, le théorème de Kolchin affirme que tout groupe unipotent fixe un vecteur non trivial. On a donc nécessairement $E=\mathbb{R}^{n+1}$ et $N=\{1\}$. Ainsi, $G$ est bien réductif.

Par conséquent, pour montrer que $G$ est semi-simple, il suffit de montrer que son centre est trivial. Soit $a$ un élément du centre de $G$. Comme $\Gamma$ est fortement irréductible, d'après le lemme 2.4, il possède au moins un élément hyperbolique $\gamma$. Notons $\rho^{+}$son rayon spectral. L'espace $\operatorname{propre} \operatorname{ker}\left(\gamma-\rho^{+}\right)$est donc de dimension 1 (voir le paragraphe 2D) et préservé par $a$. Par suite, $a$ possède une valeur propre $\lambda$ réelle. L'espace propre $\operatorname{ker}(a-\lambda)$ est préservé par $\Gamma$ et doit donc être trivial. On en déduit que $a$ est une homothétie. Comme $a \in \Gamma \subset \mathrm{SL}_{n+1}(\mathbb{R})$, on conclut que $a=1$ ou $a=-1$.

6B. Lemme de fermeture et conséquences. Nous rappelons ici un résultat classique pour les flots d'Anosov et une de ses conséquences, qui nous servira dans la partie suivante. Yves Coudene et Barbara Schapira [2010] on donné une démonstration de ces deux résultats dans le cadre de la courbure négative (ou nulle), qui s'adapte sans changement aucun. On pourra aussi consulter [Eberlein 1996] en ce qui concerne le lemme de fermeture :

1. C'est-à-dire que tout sous-groupe unipotent distingué est réduit à l'identité. 
Lemme 6.6 [Coudene et Schapira 2010, Appendice; Eberlein 1996, Proposition 4.5.15]. Soient $M=\Omega / \Gamma$ une variété quotient, avec $\Gamma$ non élémentaire, et $K$ une partie compacte de HM. Fixons $\varepsilon>0$.

Il existe $\delta>0$ et $T>0$ tels que, si $w \in K$ satisfait $d\left(w, \varphi^{t}(w)\right)<\delta$ pour un certain $t>T$, alors il existe un point $w^{\prime} \in H M$ tel que :

- $w^{\prime}$ est périodique de période $t^{\prime} \in(t-\varepsilon, t+\varepsilon)$;

- pour tout $0<s<\min \left\{t, t^{\prime}\right\}, d\left(\varphi^{s}(w), \varphi^{s}\left(w^{\prime}\right)\right)<\varepsilon$.

En version courte, cela signifie que si un point revient assez proche de sa position d'origine après un temps $t$, alors il existe une orbite périodique qui suit son orbite pendant le temps $t$, et ce en restant aussi proche qu'on le veut.

Prenons une variété quotient $M=\Omega / \Gamma$, avec $\Gamma$ non élémentaire. Notons $M$ l'ensemble des mesures de probabilité sur NW invariantes par le flot, qu'on munit de la convergence étroite des mesures : une suite $\left(\eta_{n}\right)$ de $\mu$ converge vers $\eta$ si, pour toute fonction $f$ continue sur NW, $\int f d \eta_{n}$ converge vers $\int f d \eta$. L'ensemble $M$ est un convexe dont les points extrémaux sont les mesures ergodiques. Parmi les mesures ergodiques, on peut distinguer le sous-ensemble $M_{\text {Per }}$ constitué des mesures de Lebesgue portées par les orbites périodiques. Le lemme de fermeture entraîne :

Proposition 6.7 [Coudene et Schapira 2010, Corollaire 2.3]. Soit $M=\Omega / \Gamma$ une variété quotient, avec $\Gamma$ non élémentaire. L'enveloppe convexe de $M_{\mathrm{Per}}$ est dense dans $M$.

\section{Régularité du bord}

Définition 7.1. On dira qu'un point $w=(x,[\xi]) \in H \Omega$, ou le rayon géodésique $\left\{\pi \varphi^{t}(w)\right\}_{t \geqslant 0}$ qu'il définit, est hyperbolique si, pour tout vecteur stable $Z^{s} \in E^{s}(w)$, on a

$$
\limsup _{t \rightarrow+\infty} \frac{1}{t} \ln \left\|d \varphi^{t} Z^{s}\right\|<0 .
$$

Si $w$ est un point hyperbolique, il existe alors $\chi>0$ tel que, pour tout vecteur stable $Z^{s} \in E^{s}(w)$,

$$
\limsup _{t \rightarrow+\infty} \frac{1}{t} \ln \left\|d \varphi^{t} Z^{s}\right\| \leqslant-\chi
$$

Pour tout $w \in H \Omega$, on notera $\chi(w) \geqslant 0$ le plus grand des réels $\chi$ qui vérifie l'inégalité précédente pour tout vecteur $Z^{s} \in E^{s}(w)$; autrement dit,

$$
\chi(w)=\inf _{Z^{s} \in E^{s}(w)} \liminf _{t \rightarrow+\infty}-\frac{1}{t} \ln \left\|d \varphi^{t} Z^{s}\right\| .
$$

Le point $w$ est donc hyperbolique si et seulement si $\chi(w)>0$. 
En fait, on peut facilement caractériser les points de $H \Omega$ qui sont hyperboliques, et même déterminer $\chi(w)$, selon la régularité du bord $\partial \Omega$ au point extrémal $x^{+} \mathrm{du}$ rayon défini par $w$.

Proposition 7.2. Un point $w \in H \Omega$ est hyperbolique, de coefficient $\chi(w)>0$, si et seulement si $\partial \Omega$ est de classe $\mathscr{b}^{1+\varepsilon}$ en $x^{+}$, pour tout $0<\varepsilon<(2 / \chi(w)-1)^{-1}$.

Démonstration. Reprenons les notations du lemme 3.1 : on a choisi une carte adaptée au point $w \in H \Omega, Z$ est un vecteur stable tangent à $H \Omega$ au point $w$, et $z=d \pi(Z)$, $z_{t}=\left(d \pi d \varphi^{t} Z\right)$. Tout se passe dans un plan et on peut donc supposer qu'on est en dimension 2. On a vu durant la démonstration du lemme 3.1 que

$$
\left\|d \varphi^{t}(Z)\right\|=F\left(z_{t}\right)=\frac{|z|}{2\left|x x^{+}\right|}\left(\frac{\left|x_{t} x^{+}\right|}{\left|x_{t} y_{t}^{+}\right|}+\frac{\left|x_{t} x^{+}\right|}{\left|x_{t} y_{t}^{-}\right|}\right) .
$$

Ainsi, le rayon géodésique défini par $w$ est hyperbolique si et seulement si

$$
\liminf _{t \rightarrow+\infty} \frac{1}{t} \ln \left(\frac{\left|x_{t} x^{+}\right|}{\left|x_{t} y_{t}^{+}\right|}+\frac{\left|x_{t} x^{+}\right|}{\left|x_{t} y_{t}^{-}\right|}\right)<0 ;
$$

autrement dit si et seulement s'il existe $\chi, C>0$ tels que pour $t \geqslant 0$,

soit

$$
\frac{\left|x_{t} x^{+}\right|}{\left|x_{t} y_{t}^{+}\right|}+\frac{\left|x_{t} x^{+}\right|}{\left|x_{t} y_{t}^{-}\right|}<C e^{-\chi t}
$$

$$
\frac{\left|x_{t} x^{+}\right|}{\left|x_{t} y_{t}^{+}\right|}<C e^{-\chi t} \text { et } \quad \frac{\left|x_{t} x^{+}\right|}{\left|x_{t} y_{t}^{-}\right|}<C e^{-\chi t} \text {. }
$$

Mais de l'égalité $d_{\Omega}\left(x, x_{t}\right)=t=\frac{1}{2} \ln \left[x^{+}: x^{-}: x: x_{t}\right]$, on tire

$$
\left|x_{t} x^{+}\right|=e^{-2 t} \frac{\left|x_{t} x^{-}\right|}{\left|x x^{-}\right|}\left|x x^{+}\right|,
$$

et donc il existe une constante $C_{0} \geqslant 1$ pour laquelle

$$
C_{0}^{-1} e^{-2 t} \leqslant\left|x_{t} x^{+}\right| \leqslant C_{0} e^{-2 t} .
$$

Ainsi, le rayon géodésique défini par $w$ est hyperbolique si et seulement s'il existe $\chi, D>0$ tels que pour $t \geqslant 0$,

soit

$$
\frac{\left|x_{t} x^{+}\right|}{\left|x_{t} y_{t}^{+}\right|}<D\left|x_{t} x^{+}\right|^{x / 2} \text { et } \frac{\left|x_{t} x^{+}\right|}{\left|x_{t} y_{t}^{-}\right|}<D\left|x_{t} x^{+}\right|^{\chi / 2}
$$

$$
\left|x_{t} x^{+}\right|^{1-\chi / 2}<D\left|x_{t} y_{t}^{+}\right| \text {et }\left|x_{t} x^{+}\right|^{1-\chi / 2}<D\left|x_{t} y_{t}^{-}\right| .
$$

Appelons $f: T_{x^{+}} \partial \Omega \mapsto \mathbb{R}$ le graphe de $\partial \Omega$, de telle façon que

$$
\left|x_{t} x^{+}\right|=f\left(\left|x_{t} y_{t}^{+}\right|\right)=f\left(-\left|x_{t} y_{t}^{-}\right|\right) .
$$


La condition précédente est alors équivalente à : pour tout $s$ (assez petit),

$$
f(s)<D s^{1 /(1-\chi / 2)} .
$$

Or, dans notre carte adaptée, on a $d_{x^{+}} f=0$. L'inégalité précédente veut donc dire que la fonction $f$ est $\mathscr{b}^{1+\varepsilon}$ en $x^{+}$avec $\varepsilon=1 /(2 / \chi-1)$.

Cette proposition et le théorème 5.2 ont la conséquence suivante :

Corollaire 7.3. Supposons que $\Omega$ admette une action géométriquement finie à pointes asymptotiquement hyperboliques d'un sous-groupe discret $\Gamma$ de $\operatorname{Aut}(\Omega)$. Alors il existe $\varepsilon>0$ tel que le bord $\partial \Omega$ de $\Omega$ est de classe $\mathscr{C}^{1+\varepsilon}$ en tout point de $\Lambda_{\Gamma}$.

En particulier, si $\Omega$ admet une action de covolume fini, alors le bord $\partial \Omega$ de $\Omega$ est de classe $\mathscr{C}^{1+\varepsilon}$ pour un certain $\varepsilon>0$.

7A. Régularité optimale du bord. Soit $\Gamma$ un sous-groupe discret de $\operatorname{Aut}(\Omega)$. Notons

$$
\varepsilon\left(\Lambda_{\Gamma}\right)=\sup \left\{\varepsilon \in[0,1] \mid \text { le bord } \partial \Omega \text { est } \mathscr{C}^{1+\varepsilon} \text { en tout point de } \Lambda_{\Gamma}\right\} .
$$

Pour tout élément hyperbolique $\gamma \in \Gamma$, notons

$$
\varepsilon(\gamma)=\sup \left\{\varepsilon \in[0,1] \mid \text { le bord } \partial \Omega \text { est } \mathscr{C}^{1+\varepsilon} \text { en } x_{\gamma}^{+}\right\},
$$

et $\varepsilon(\Gamma)=\inf \{\varepsilon(\gamma) \mid \gamma \in \Gamma$ hyperbolique $\}$. Le bord $\partial \Omega$ est ainsi de classe $\mathscr{b}^{1+\varepsilon(\Gamma)}$ en tout point fixe d'un élément hyperbolique de $\Gamma$. Rappelons-nous que l'ensemble des points fixes d'éléments hyperboliques de $\gamma$ est dense dans $\Lambda_{\Gamma}$, dès que $\Gamma$ n'est pas élémentaire; on pourrait donc s'attendre au théorème qui suit, qui est toutefois faux en général.

Théorème 7.4. Supposons que $\Omega$ admette une action géométriquement finie à pointes asymptotiquement hyperboliques $\Gamma$ d'un sous-groupe discret $\Gamma$ de $\operatorname{Aut}(\Omega)$. Alors

$$
\varepsilon\left(\Lambda_{\Gamma}\right)=\varepsilon(\Gamma) .
$$

Dans le cas où le groupe $\Gamma$ est cocompact, l'ensemble limite $\Lambda_{\Gamma}$ est tout le bord $\partial \Omega$ et ce résultat a déjà été prouvé par Olivier Guichard. Nous allons donner ici une toute autre démonstration. Toutefois, remarquons que la méthode de Guichard est plus précise car elle permet de prouver que le supremum dans la définition (7-1) est en fait un maximum, c'est-à-dire que $\partial \Omega$ est exactement $\mathscr{C}^{1+\varepsilon(\Gamma)}$ (et pas plus, sauf si $\Omega$ est un ellipsoïde).

Remarquons que l'hypothèse faite sur les pointes est essentielle. En effet, il est possible de faire en sorte que la régularité en un point parabolique soit aussi mauvaise que l'on veut car lorsque le groupe parabolique n'est pas de rang maximal, il n'impose la régularité du bord au point fixe que dans certaines directions. On consultera la partie 8.1 à ce propos. 
Notre démonstration repose sur une approche dynamique et en particulier sur l'extension d'un résultat de Ursula Hamenstädt concernant le "meilleur" coefficient de contraction du flot géodésique [Hamenstädt 1994]. Au vu de la proposition 7.2, cette approche est en fait totalement naturelle. Dans toute la suite, nous supposerons que $\Gamma$ est sans torsion, ce qui ne change rien d'après le lemme de Selberg, et nous étudierons plus en détail le flot géodésique de $M=\Omega / \Gamma$.

Rappelons que l'ensemble non errant NW du flot géodésique sur $H M$ est la projection sur $H M$ de l'ensemble

$$
\left\{w=(x, \xi) \in H \Omega \mid x^{+}, x^{-} \in \Lambda_{\Gamma}\right\} .
$$

Notons $\chi(\mathrm{NW})$ la meilleure constante d'hyperbolicité du flot géodésique sur l'ensemble non errant; autrement dit, $\chi(\mathrm{NW})$ est le supremum des réels $\chi \geqslant 0$ tels qu'il existe $C>0$ tel que, pour tout $w \in \mathrm{NW}$ et tous $Z^{s} \in E^{s}(w), Z^{u} \in E^{u}(w)$, on a

$$
\left\|d \varphi^{t}\left(Z^{s}\right)\right\| \leqslant C e^{-\chi t}\left\|Z^{s}\right\|, \quad\left\|d \varphi^{-t}\left(Z^{u}\right)\right\| \leqslant C e^{-\chi t}\left\|Z^{u}\right\|, \quad t \geqslant 0 .
$$

On a en fait

$$
\chi(\mathrm{NW})=\inf _{w \in \mathrm{NW}} \chi(w) .
$$

On a déjà vu que les points périodiques formaient un ensemble Per dense dans NW. Notons

$$
\chi(\text { Per })=\inf \{\chi(w) \mid w \in \mathrm{NW} \text { périodique }\} .
$$

Le résultat, inspiré de celui d'Hamenstädt, est le suivant :

Théorème 7.5. Soit $M=\Omega / \Gamma$ une variété géométriquement finie à pointes asymptotiquement hyperboliques. On a

$$
\chi(\mathrm{NW})=\chi(\text { Per })
$$

Voyons tout de suite comment ce dernier résultat implique directement le théorème 7.4. Rappelons que l'ensemble des orbites périodiques est en bijection avec les classes de conjugaison d'éléments hyperboliques de $\Gamma:$ si $\gamma \in \Gamma$ est hyperbolique, la projection de la géodésique orientée $\left(x_{\gamma}^{-} x_{\gamma}^{+}\right)$sur $H M$ est une orbite périodique du flot géodésique, qu'on note encore $\gamma$. Associé à cette orbite $\gamma$, on dispose du plus petit coefficient de contraction :

$$
\chi(\gamma):=\inf _{Z^{s} \in E^{s}(w)} \liminf _{t \rightarrow+\infty}-\frac{1}{t} \ln \left\|d \varphi^{t} Z^{s}\right\|=\chi(w),
$$

où $w$ est un point quelconque de l'orbite $\gamma$. L'égalité principale est la suivante, qui découle de la proposition 7.2 :

$$
\varepsilon(\Gamma)=\frac{1}{2 / \chi(\text { Per })-1} .
$$


Il s'avère qu'on peut exprimer $\chi(\gamma)$ en fonction des valeurs propres de $\gamma$, comme l'affirme le lemme suivant, montré dans [Benoist 2004] ou dans [Crampon 2009] :

Lemme 7.6. Soit $\gamma \in \operatorname{Aut}(\Omega)$ un élément hyperbolique. Notons

$$
\lambda_{0}(\gamma) \geqslant \lambda_{1}(\gamma) \geqslant \cdots \geqslant \lambda_{n}(\gamma)>0
$$

les modules de ses valeurs propres, comptées avec multiplicité. Alors

$$
\chi(\gamma)=2\left(1-\frac{\ln \lambda_{0}(\gamma)-\ln \lambda_{n-1}(\gamma)}{\ln \lambda_{0}(\gamma)-\ln \lambda_{n}(\gamma)}\right) .
$$

Corollaire 7.7. Pour toute variété quotient $M=\Omega / \Gamma$, on a $\chi($ Per $) \leqslant 1$. De plus, si $\chi($ Per $)=1$, alors $\Gamma$ n'est pas Zariski-dense dans $\mathrm{SL}_{n+1}(\mathbb{R})$.

Démonstration. En gardant les notations du lemme, on voit que si $\gamma \in \operatorname{Aut}(\Omega)$ est hyperbolique, alors

$$
\chi\left(\gamma^{-1}\right)=2\left(1-\frac{\ln \lambda_{1}(\gamma)-\ln \lambda_{n}(\gamma)}{\ln \lambda_{0}(\gamma)-\ln \lambda_{n}(\gamma)}\right) .
$$

Ainsi,

$$
\chi(\gamma)+\chi\left(\gamma^{-1}\right)=2\left(1-\frac{\ln \lambda_{1}(\gamma)-\ln \lambda_{n-1}(\gamma)}{\ln \lambda_{0}(\gamma)-\ln \lambda_{n}(\gamma)}\right) \leqslant 2 .
$$

Cela implique que soit $\chi(\gamma) \leqslant 1$ soit $\chi\left(\gamma^{-1}\right) \leqslant 1$, et donc que

$$
\chi(\text { Per })=\inf \{\chi(\gamma) \mid \gamma \in \Gamma \text { hyperbolique }\} \leqslant 1 .
$$

Maintenant, supposons que $\chi($ Per $)=1$, c'est-à-dire que $\chi(\gamma) \geqslant 1$ pour tout élément $\gamma \in \Gamma$ hyperbolique. De l'inégalité $\chi(\gamma)+\chi\left(\gamma^{-1}\right) \leqslant 2$, on déduit que $\chi(\gamma)=1$ pour tout élément $\gamma \in \Gamma$ hyperbolique. D'après le lemme précédent, cela veut dire que, pour tout élément $\gamma \in \Gamma$ hyperbolique,

soit

$$
2\left(1-\frac{\ln \lambda_{0}(\gamma)-\ln \lambda_{1}(\gamma)}{\ln \lambda_{0}(\gamma)-\ln \lambda_{n}(\gamma)}\right)=1
$$

$$
\ln \lambda_{0}(\gamma)+\ln \lambda_{n}(\gamma)-2 \ln \lambda_{1}(\gamma)=0
$$

En particulier, l'ensemble $\ln (\Gamma)$ n'engendre pas tout l'espace

$$
\ln \left(\mathrm{SL}_{n+1}(\mathbb{R})\right)=\left\{x=\left(x_{0}, \cdots, x_{n}\right) \in \mathbb{R}^{n+1} \mid \sum_{i=0}^{n} x_{i}=0\right\} .
$$

D'après le théorème 6.4, cela implique que $\Gamma$ n'est pas Zariski-dense dans $\operatorname{SL}_{n+1}(\mathbb{R})$. 
Démonstration du théorème 7.4. D'après l'égalité (7-2), on a

$$
\varepsilon(\gamma)=\min \left(1, \frac{1}{2 / \chi(\gamma)-1}\right)
$$

et donc, via le corollaire 7.7, que

$$
\varepsilon(\Gamma)=\frac{1}{2 / \chi(\text { Per })-1} .
$$

De même, on a

Le théorème 7.5 implique

$$
\varepsilon\left(\Lambda_{\Gamma}\right)=\frac{1}{2 / \chi(\mathrm{NW})-1} .
$$

$$
\varepsilon\left(\Lambda_{\Gamma}\right)=\varepsilon(\Gamma) .
$$

7B. Conséquences. La régularité optimale du bord aux points de $\Lambda_{\Gamma}$ se lit donc sur les valeurs propres des éléments de $\Gamma$ :

Théorème 7.8. Supposons que $\Omega$ admette une action géométriquement finie à pointes asymptotiquement hyperboliques d'un sous-groupe discret $\Gamma$ de $\operatorname{Aut}(\Omega)$. Alors

$$
\varepsilon\left(\Lambda_{\Gamma}\right)=\inf \left\{\frac{\ln \lambda_{n-1}(\gamma)-\ln \lambda_{n}(\gamma)}{\ln \lambda_{0}(\gamma)-\ln \lambda_{n-1}(\gamma)} \mid \gamma \in \Gamma \text { hyperbolique }\right\} .
$$

Démonstration. C'est un simple calcul à partir de l'égalité

$$
\varepsilon\left(\Lambda_{\Gamma}\right)=\frac{1}{2 / \chi(\text { Per })-1} .
$$

Les corollaires ci-dessous sont sûrement plus parlants, et sont complémentaires du résultat de rigidité de Benoist concernant la régularité des convexes divisibles [Benoist 2004, Proposition 6.1]. Il repose sur le résultat suivant, cas particulier d'un des théorèmes principaux de [Crampon et Marquis 2012] :

Théorème 7.9. Soit $\Gamma$ un sous-groupe discret de Aut $(\Omega)$. Si $\Gamma$ agit de façon géométriquement finie sur $\Omega$ et contient un élément parabolique, alors son adhérence de Zariski est soit $\mathrm{SL}_{n+1}(\mathbb{R})$ tout entier, soit conjuguée à $\mathrm{SO}_{n, 1}(\mathbb{R})$.

Corollaire 7.10. Supposons que $\Omega$ admette une action géométriquement finie d'un sous-groupe discret $\Gamma$ de $\operatorname{Aut}(\Omega)$ qui contienne un élément parabolique. Si le bord $\partial \Omega$ est de classe $\mathscr{C}^{1+\varepsilon}$ pour tout $0<\varepsilon<1$ en tout point de $\Lambda_{\Gamma}$, alors $\Gamma$ est conjugué à un sous-groupe de $\mathrm{SO}_{n, 1}(\mathbb{R})$.

Démonstration. Les hypothèses impliquent que $\varepsilon(\Gamma)=1$, soit $\chi($ Per $)=1$. Le corollaire 7.7 implique que $\Gamma$ n'est pas Zariski-dense dans $\mathrm{SL}_{n+1}(\mathbb{R})$. D'après le théorème précédent, $\Gamma$ est Zariski-dense dans un conjugué de $\mathrm{SO}_{n, 1}(\mathbb{R})$.

Un cas particulier est le suivant, où l'on obtient une vraie rigidité : 
Corollaire 7.11. Si $\Omega$ admet un quotient de volume fini non compact, alors le bord $\partial \Omega$ est de classe $\mathscr{C}^{1+\varepsilon}$ pour tout $0<\varepsilon<1$ si et seulement si $\Omega$ est un ellipsoïde.

7C. Démonstration du théorème 7.5. Cette démonstration, largement inspirée de [Hamenstädt 1994], est assez technique. En voici d'abord le schéma, qui repousse la partie la plus délicate, incluse dans le lemme 7.12, à la suite.

Démonstration. Notons pour simplifier $\chi=\chi$ (Per). Cela veut dire que pour tout $\varepsilon>0$ et point $w \in \mathrm{NW}$ périodique, il existe une constante $C_{\varepsilon}(w)$ telle que, pour tout vecteur tangent stable $Z$ en $w$, on ait

$$
\left\|d \varphi^{t} Z\right\| \leqslant C_{\varepsilon}(w) e^{-(\chi-\varepsilon) t}\|Z\| .
$$

Considérons l'ensemble

$$
A_{T, \varepsilon}=\left\{w \in \mathrm{NW} \mid \frac{\left\|d \varphi^{T} Z\right\|}{\|Z\|} \leqslant e^{-(\chi-\varepsilon) T} \text { pour chaque } Z \in E^{S}(w)\right\} .
$$

Un point $w$ n'est pas dans $A_{T, \varepsilon}$ s'il existe un vecteur stable en $w$ qui est contracté $\operatorname{par} \varphi^{T}$ avec un exposant inférieur à $\chi-\varepsilon$. En particulier, à $\varepsilon$ fixé, pour tout point $w$ périodique, il existe un temps $T(w)$ tel que pour tout $t \geqslant T(w), w \in A_{t, \varepsilon}$. On va montrer qu'en fait l'orbite de tout point $w \in \mathrm{NW}$ sous $\varphi^{T}$ passe "la plupart du temps" dans $A_{T, \varepsilon}$ si $T$ est assez grand. Pour cela on pose

$$
N_{n, T, \varepsilon}=\frac{1}{n} \sum_{i=0}^{n-1} \mathbf{1}_{A_{T, \varepsilon}}\left(\varphi^{i T} w\right) .
$$

$N_{n, T, \varepsilon}$ compte la proportion des $n$ premiers points de l'orbite de $w$ sous $\varphi^{T}$ qui sont dans $A_{T, \varepsilon}$. Le résultat principal est le suivant :

Lemme 7.12. Pour tous $\varepsilon, \delta>0$, il existe $T=T(\varepsilon, \delta)$ et $N=N(T)$ tels que, pour $n \geqslant N$ et $w \in \mathrm{NW}$,

$$
N_{n, T, \varepsilon}(w) \geqslant 1-\delta .
$$

Le théorème découle aisément de ce lemme. En effet, pour tous $w \in \mathrm{NW}$ et $Z \in E^{s}(w)$, on a

$$
\begin{aligned}
\frac{\left\|d \varphi^{n T} Z\right\|}{\|Z\|} & \leqslant \prod_{i=0}^{n-1} \frac{\left\|d \varphi^{(i+1) T} Z\right\|}{\left\|d \varphi^{i T} Z\right\|} \\
& \leqslant \prod_{\substack{0 \leqslant i \leqslant n-1 \\
\varphi^{i T} w \notin A_{t, \varepsilon}}} \frac{\left\|d \varphi^{(i+1) T} Z\right\|}{\left\|d \varphi^{i T} Z\right\|} \prod_{\substack{0 \leqslant i \leqslant n-1 \\
\varphi^{i T} w \in A_{t, \varepsilon}}} \frac{\left\|d \varphi^{(i+1) T} Z\right\|}{\left\|d \varphi^{i T} Z\right\|} \\
& \leqslant 1 \cdot e^{-(\chi-\varepsilon) N_{n, T, \varepsilon}(w) n T} .
\end{aligned}
$$


La majoration par 1 du premier terme découle du lemme 3.1. Si maintenant, à $\varepsilon$ et $\delta$ fixés, on prend $T \geqslant T(\varepsilon, \delta)$ et $n \geqslant N(T)$, on obtient, pour tout $Z \in E^{s}$ :

$$
\frac{\left\|d \varphi^{n T} Z\right\|}{\|Z\|} \leqslant e^{-(\chi-\varepsilon)(1-\delta) n T} .
$$

En prenant $\delta=\varepsilon /(\chi-\varepsilon)$, cela donne

$$
\frac{\left\|d \varphi^{n T} Z\right\|}{\|Z\|} \leqslant e^{-(\chi-2 \varepsilon) n T} .
$$

On en conclut qu'il existe une constante $C$ telle que pour tous $Z \in E^{s}$ et $t \geqslant 0$,

$$
\frac{\left\|d \varphi^{t} Z\right\|}{\|Z\|} \leqslant C e^{-(\chi-2 \varepsilon) t} .
$$

C'est gagné, puisque $\varepsilon$ est arbitrairement petit.

Nous allons ici nous servir de la proposition 6.7 pour attaquer la partie technique : le lemme 7.12. On définit pour $k \in \mathbb{N}$ et $w \in \mathrm{NW}$,

$$
Q_{k}(w)=\inf \left\{-\frac{1}{2^{k}} \ln \frac{\left\|d \varphi^{2^{k}} Z\right\|}{\|Z\|} \mid Z \in E^{s}(w)\right\} .
$$

Comme le flot est uniformément hyperbolique, $Q_{k}$ est $\geqslant 0$ et majoré, indépendamment de $k$. Posons alors

$$
F_{k, \varepsilon}=\max \left\{0,(\chi-\varepsilon)-Q_{k}\right\} .
$$

Ainsi, $F_{k, \varepsilon}(w)>0$ s'il y a un vecteur stable en $w$ qui est contracté avec un exposant inférieur à $\chi-\varepsilon$. Autrement dit, $F_{k, \varepsilon}(w)>0$ si et seulement si $w \notin A_{2^{k}, \varepsilon}$.

Les fonctions $Q_{k}$ et $F_{k, \varepsilon}$ sont toutes deux positives, continues sur NW, et majorées indépendamment de $k$. Le premier lemme est le suivant :

Lemme 7.13. Soit $\varepsilon>0$. Pour $k$ assez grand, la fonction $F_{k, \varepsilon}$ est à support compact sur NW.

Démonstration. Rappelons-nous que le coeur convexe $C(M)$ de $M$ se décompose en une partie compacte $K$ et un nombre fini de pointes $\mathscr{C}_{i}, 1 \leqslant i \leqslant l$, asymptotiquement hyperboliques. On a montré (voir la remarque 5.7) que pour tout point $w \in H M$ tel que $\varphi^{s}(w) \in H^{\mathscr{C}} \mathfrak{C}_{i}, 0 \leqslant s \leqslant t$, on avait

$$
\left\|d \varphi^{t} Z^{s}\right\| \leqslant M e^{-t}\left\|Z^{s}\right\|, \quad Z^{s} \in E^{s}(w),
$$

pour une certaine constante $M>0$. Ainsi, pour tout $\delta>0$, il existe $T_{\delta}$ tel que pour $t \geqslant T_{\delta}$, on a, pour tout point $w \in H M$ tel que $\varphi^{s}(w) \in H \mathscr{C}_{i}, 0 \leqslant s \leqslant t$,

$$
\left\|d \varphi^{t} Z^{s}\right\| \leqslant e^{-(1-\delta) t}\left\|Z^{s}\right\|, \quad Z^{s} \in E^{s}(w) .
$$


Comme d'après le corollaire 7.7, on a toujours $\chi \leqslant 1$, on peut prendre

$$
\delta=1-\frac{1+\chi-\varepsilon}{2}
$$

et $k$ tel que $2^{k} \geqslant T_{\delta}$. On obtient alors que, pour tout point $w \in H M$ tel que $\varphi^{s}(w) \in H \mathscr{b}_{i}, 0 \leqslant s \leqslant 2^{k}$,

$$
\left\|d \varphi^{2^{k}} Z^{s}\right\| \leqslant e^{-\frac{1+\chi-\varepsilon}{2} 2^{k}}\left\|Z^{s}\right\|, \quad Z^{s} \in E^{s}(w) .
$$

En particulier, $F_{k, \varepsilon}=0$ sur l'ensemble

$$
\left\{w \in \mathrm{NW} \mid \varphi^{s}(w) \in H^{\mathscr{C}_{i}}, 0 \leqslant s \leqslant 2^{k}\right\},
$$

dont le complémentaire dans NW est compact. La fonction $F_{k, \varepsilon}$ est donc à support compact pour $k$ assez grand.

Rappelons qu'on a noté $\mathcal{M}$ l'ensemble des mesures de probabilités sur NW invariantes par le flot.

Lemme 7.14. Pour tous $\varepsilon, \delta>0$, il existe $k(\varepsilon, \delta)$ tel que pour tout $k \geqslant k(\varepsilon, \delta)$ et tout $\eta \in \mathcal{M}$,

$$
\int F_{k, \varepsilon} d \eta<\delta \text {. }
$$

Démonstration. Fixons $\varepsilon>0$, et choisissons $k$ assez grand pour que la fonction $F_{k, \varepsilon}$ soit à support compact $S$ sur NW. Notons $\mathfrak{M}$ l'espace vectoriel des mesures de Radon signées sur NW, muni de la topologie de la convergence étroite des mesures. Si $A$ est un compact de NW et $m>0$, l'ensemble des mesures de $\mathfrak{M}$ à support dans $A$ et de masse totale $\leqslant m$ est compact pour cette topologie. En particulier, en notant, pour $\eta \in \mathfrak{M}, \eta_{S}$ la mesure définie par

$$
\eta_{S}(B)=\eta(S \cap B), \quad B \text { Borélien de NW, }
$$

l'ensemble

$$
\mathcal{M}(S)=\left\{\eta_{S} \mid \eta \in \mathcal{M}\right\}
$$

est un ensemble compact.

On définit une forme linéaire sur $\mathfrak{M}$ par

$$
\Psi_{k}: \eta \in \mathfrak{M} \mapsto \int F_{k, \varepsilon} d \eta \in \mathbb{R} .
$$

Remarquons tout de suite que $\Psi_{k}(\eta)=\Psi_{k}\left(\eta_{S}\right)$ pour toute mesure $\eta \in \mathfrak{M}$. La forme linéaire $\Psi_{k}$ est positive et continue, et surtout la suite $\left(\Psi_{k}\right)$ est uniformément bornée : pour tout $k$,

$$
\left\|\Psi_{k}\right\|=\sup _{\|\eta\| \leqslant 1}\left|\Psi_{k}(\eta)\right| \leqslant\left\|F_{k, \varepsilon}\right\|_{\infty}<\chi<+\infty .
$$

On munit l'espace $\mathfrak{M}^{\prime}$ des formes linéaires continues sur $\mathfrak{M}$ de la topologie 
*-faible : une suite $\left(\Phi_{n}\right)$ de $\mathfrak{M}^{\prime}$ converge vers $\Phi$ si pour toute mesure $\eta \in \mathfrak{M},\left(\Phi_{n}(\eta)\right)$ converge vers $\Phi(\eta)$. Pour cette topologie, les ensembles bornés sont relativement compacts. En particulier, on peut supposer, quitte à extraire une sous-suite, que la suite $\left(\Psi_{k}\right)$ converge vers $\Psi \in \mathfrak{M}^{\prime}$.

Notons $C\left(M_{\mathrm{Per}}\right)$ l'enveloppe convexe de l'ensemble $M_{\text {Per }}$ des mesures portées par les orbites périodiques. Si $\eta \in C\left(M_{\mathrm{Per}}\right)$, on a, par construction, $\Psi(\eta)=0$. Par densité de $C\left(M_{\mathrm{Per}}\right)$ dans $\mathcal{M}$ (proposition 6.7) et continuité de $\Psi$, on en déduit que $\Psi=0$ sur $M$. Maintenant, en écrivant, pour $Z \in E^{s}$,

$$
-\frac{1}{2^{k+1}} \ln \frac{\left\|d \varphi^{2^{k+1}} Z\right\|}{\|Z\|}=\frac{1}{2}\left(-\frac{1}{2^{k}} \ln \frac{\left\|d \varphi^{2^{k}} d \varphi^{2^{k}} Z\right\|}{\left\|d \varphi^{2^{k}} Z\right\|}-\frac{1}{2^{k}} \ln \frac{\left\|d \varphi^{2^{k}} Z\right\|}{\|Z\|}\right),
$$

on remarque facilement que

$$
Q_{k+1} \geqslant \frac{1}{2}\left(Q_{k} \circ \varphi^{2^{k}}+Q_{k}\right) .
$$

De là, on obtient par définition de $F_{k, \varepsilon}$ que

$$
F_{k+1, \varepsilon} \leqslant \frac{1}{2}\left(F_{k, \varepsilon} \circ \varphi^{2^{k}}+F_{k, \varepsilon}\right) .
$$

Cela entraîne que, si $\eta \in \mathcal{M}$ est une mesure de probabilité invariante,

$\Psi_{k+1}(\eta)=\int F_{k+1, \varepsilon} d \eta \leqslant \frac{1}{2}\left(\int F_{k, \varepsilon} \circ \varphi^{2^{k}} d \eta+\int F_{k, \varepsilon} d \eta\right)=\int F_{k, \varepsilon} d \eta=\Psi_{k}(\eta)$.

Ainsi, pour tout $\eta \in \mathcal{M}$, la suite $\left(\Psi_{k}(\eta)\right)$ est décroissante.

Ainsi, la suite de fonctions $\Psi_{k}: M \rightarrow \mathbb{R}$ converge en décroissant vers 0 . L'ensemble $M(S)$ étant compact, le théorème de Dini entraîne que la convergence de $\left(\Psi_{k}\right)$ vers 0 est uniforme sur $\mathcal{M}(S)$, donc sur $\mathcal{M}$ puisque $\Psi_{k}(\eta)=\Psi_{k}\left(\eta_{S}\right)$ pour tout $\eta \in \mathcal{M}$. Autrement dit, pour tout $\delta>0$, il existe $k(\varepsilon, \delta)$ tel que pour $k \geqslant k(\varepsilon, \delta)$ et $\eta \in \mathcal{M}$,

$$
\Psi_{k}(\eta)=\int F_{k, \varepsilon} d \eta \leqslant \delta .
$$

Nous aurons enfin besoin de l'observation élémentaire qui suit :

Lemme 7.15. Soient $\varepsilon>0$ et $T>0$. Si $w \notin A_{T, \varepsilon}$, alors, pour tout $-\varepsilon / 8<\lambda<\varepsilon / 8$, $\varphi^{\lambda T}(w) \notin A_{T, \varepsilon / 2}$.

Démonstration. Soit $\varepsilon>0$. En vertu du corollaire 3.2, pour tout $t \geqslant 0$ et tout $Z \in E^{s}$,

$$
\frac{\left\|d \varphi^{t} Z\right\|}{\|Z\|} \geqslant e^{-2 t}
$$

Ainsi, pour tous $T>0, \lambda \in \mathbb{R}$,

$$
\frac{\left\|d \varphi^{(1+\lambda) T} Z\right\|}{\|Z\|}=\frac{\left\|d \varphi^{\lambda T} d \varphi^{T} Z\right\|}{\left\|d \varphi^{T} Z\right\|} \frac{\left\|d \varphi^{T} Z\right\|}{\|Z\|} \geqslant e^{-2 \lambda T} \frac{\left\|d \varphi^{T} Z\right\|}{\|Z\|} .
$$


Par conséquent, si $w \notin A_{T, \varepsilon}$, alors, pour tout $\lambda \geqslant 0$ et tout $Z \in E^{s}\left(\varphi^{t} w\right)$,

$$
\begin{aligned}
\frac{\left\|d_{\varphi^{t} w} \varphi^{T} Z\right\|}{\|Z\|} & =\frac{\left\|d_{w} \varphi^{T+t} d_{\varphi^{t} w} \varphi^{-t} Z\right\|}{\left\|d_{\varphi^{t} w} \varphi^{-t} Z\right\|} \frac{\left\|d_{\varphi^{t} w} \varphi^{-t} Z\right\|}{\|Z\|} \\
& \geqslant e^{-2 \lambda T} \frac{\left\|d_{w} \varphi^{T} d_{\varphi^{t} w} \varphi^{-t} Z\right\|}{\left\|d_{\varphi^{t} w} \varphi^{-t} Z\right\|} e^{-2 \lambda T} \\
& \geqslant e^{-4 \lambda T} e^{-(\chi-\varepsilon) T} \geqslant e^{-(\chi-\varepsilon+4 \lambda) T} .
\end{aligned}
$$

Cela implique que, si $w \notin A_{T, \varepsilon}$, alors $\varphi^{\lambda T}(w) \notin A_{T, \varepsilon / 2}$ pour tout $-\frac{\varepsilon}{8}<\lambda<\frac{\varepsilon}{8}$.

Démonstration du lemme 7.12. Fixons pour la suite $\varepsilon>0$. Choisissons $\delta>0$ et $T=2^{k}$ avec $k \geqslant k\left(\varepsilon / 4, \delta \varepsilon^{2} / 16\right)$ donné par le lemme 7.14. On a donc, pour toute mesure de probabilité invariante $\eta$,

$$
\int F_{k, \varepsilon / 4} d \eta<\delta \frac{\varepsilon^{2}}{16}
$$

On procède par l'absurde en supposant qu'il existe un point $w \in \mathrm{NW}$ et une suite $\left(n_{j}\right)_{j \in \mathbb{N}}$ telle que $N_{n_{j}, \varepsilon, T}(w) \leqslant 1-\delta$. D'après le lemme 7.15 , à chaque fois qu'un point $\varphi^{i T}(w), i \in \mathbb{N}$, de l'orbite de $w$ sous $\varphi^{T}$ n'est pas dans $A_{T, \varepsilon}$, alors $\varphi^{t+i T}(w)$ n'est pas dans $A_{T, \varepsilon / 2}$ pour tout $-\varepsilon T / 8<t<\varepsilon T / 8$; et donc $F_{k, \varepsilon / 4}\left(\varphi^{t} w\right) \geqslant \varepsilon / 4$.

Or, parmi les points $\varphi^{i T}(w), 0 \leqslant i \leqslant n_{j}$ de l'orbite de $w$ sous $\varphi^{T}$, il y en a $N_{n_{j}, \varepsilon, T}(w) n_{j}$ qui sont dans $A_{T, \varepsilon}$; cela implique qu'entre les instants 0 et $n_{j} T$, l'orbite de $w$ n'est pas dans $A_{T, \varepsilon / 2}$ pendant au moins le temps $n_{j} T \delta \varepsilon / 4$. Autrement dit,

$$
\begin{aligned}
\frac{1}{n_{j} T} \int_{0}^{n_{j} T} F_{k, \varepsilon / 4}\left(\varphi^{t} w\right) d t & \geqslant \frac{1}{n_{j} T} \int_{0}^{n_{j} T} F_{k, \varepsilon / 4}\left(\varphi^{t} w\right)\left(1-\mathbf{1}_{A_{T, \varepsilon / 2}}\left(\varphi^{t} w\right)\right) d t \\
& \geqslant \frac{1}{n_{j} T} \frac{\varepsilon}{4} n_{j} T \delta \frac{\varepsilon}{4}=\delta \frac{\varepsilon^{2}}{16} .
\end{aligned}
$$

On définit la suite de mesures de probabilités $\left(\eta_{j}\right)_{j \in \mathbb{N}}$ par

$$
\int f d \eta_{j}=\frac{1}{n_{j} T} \int_{0}^{n_{j} T} f\left(\varphi^{t}(w)\right) d t, \quad f \in C(\mathrm{NW}) .
$$

Toute valeur d'adhérence $\eta$ de la suite $\left(\eta_{j}\right)$ vérifie

$$
\int F_{k, \varepsilon / 4} d \eta \geqslant \delta \frac{\varepsilon^{2}}{16} .
$$

Or, une telle mesure $\eta$ est nécessairement invariante par le flot, et cela contredit le choix de $k$. 


\section{Quelques exemples}

8A. Un exemple où le flot géodésique n'est pas uniformément hyperbolique. On va construire un exemple "dégénéré" où le flot géodésique a un exposant de Lyapunov nul. Pour cela, on considère un certain groupe fuchsien $\Gamma$ qui contient un parabolique, et on le fait agir de façon canonique sur $\mathbb{\boxplus}^{3}$; on construit alors un nouvel ouvert convexe $\Gamma$-invariant dont le bord n'est de classe $\mathscr{C}^{1+\varepsilon}$ en aucun point parabolique, pour tout $\varepsilon>0$. D'après la proposition 7.2 , toute orbite ultimement incluse dans la pointe aura un exposant de Lyapunov nul.

Le groupe $\Gamma$ ici présenté n'est donc pas irréductible sur $\mathbb{P}^{3}$ mais il est sans doute possible de le déformer par pliage, tout en préservant les propriétés de régularité que l'on désirait.

Le résultat s'énonce ainsi :

Proposition 8.1. Il existe un ouvert proprement convexe $\Omega$ de $\mathbb{P}^{3}$, strictement convexe à bord $\mathscr{C}^{1}$, et un sous-groupe discret $\Gamma$ de $\operatorname{Aut}(\Omega)$ tels que:

- l'action de $\Gamma$ sur $\Omega$ soit géométriquement finie mais non convexe-cocompacte;

- le bord $\partial \Omega$ de $\Omega$ n'est pas de classe $\complement^{1+\varepsilon}$ aux points paraboliques de $\Lambda_{\Gamma}$, pour tout $\varepsilon>0$.

En particulier, le flot géodésique sur la variété quotient $\Omega / \Gamma$ a un exposant de Lyapunov nul; il n'est donc pas uniformément hyperbolique.

Démonstration. Soient $\Sigma$ le tore à 1 trou et $\Gamma$ son groupe fondamental; $\Gamma$ est un groupe libre à 2 générateurs. On munit $\Sigma$ d'une structure hyperbolique de volume

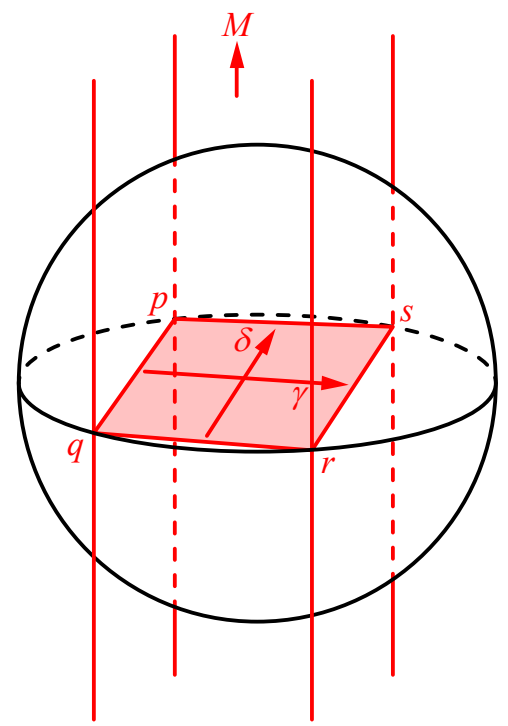

Figure 9. Domaines fondamentaux. 
fini de la façon suivante : on se donne un carré idéal $P$ de $\mathbb{H}^{2}$ et on identifie les côtés opposés de ce carré à l'aide de deux éléments hyperboliques $\gamma$ et $\delta$. Ainsi le domaine fondamental pour l'action de $\Gamma$ sur $\mathbb{W}^{2}$ est le carré idéal en question. Pour simplifier la discussion, on choisit ce carré idéal de telle façon qu'il ait un groupe diédral d'ordre 8 de symétrie.

À présent, on plonge $\Gamma$ dans $\mathrm{SO}_{3,1}(\mathbb{R})$ de façon canonique. Ainsi, $\Gamma$ agit sur l'espace hyperbolique $\mathbb{H}^{3}$ de dimension 3. L'ensemble limite de $\Gamma$ sur $\mathbb{T}^{3}$ est un cercle, intersection d'un plan projectif $\Pi$ et de $\partial \mathbb{H}^{3}$.

Le groupe $\Gamma$ préserve le plan $\Pi$ et le point $M$ intersection des hyperplans tangents à $\partial \mathbb{M}^{3}$ en $\Lambda_{\Gamma}=\Pi \cap \partial \mathbb{\boxplus}^{3}$. L'ouvert convexe $\Omega_{\infty}$ obtenu en prenant la réunion des deux cônes de bases $\Pi \cap \mathbb{H}^{3}$ et de sommet $M$ est préservé par $\Gamma$ et $\operatorname{par} \mathrm{SO}_{2,1}(\mathbb{R})$. Un domaine fondamental $D$ pour l'action de $\Gamma$ sur $\Omega_{\infty}$ est la réunion des deux cônes de base $P$ et de sommet $M$. Nous allons construire une partie convexe $D_{1}$ de $D$ telle que la réunion $\bigcup_{\gamma \in \Gamma} \gamma\left(D_{1}\right)$ nous donne l'ouvert convexe $\Omega$ désiré.

On note $p, q, r, s$ les sommets de $P, \gamma$ l'élément qui identifie [ $p q]$ avec [sr] et $\delta$ celui qui identifie $[r q]$ avec $[s p]$ (voir la figure 10 ); le groupe $\Gamma$ est engendré par ces deux éléments $\gamma$ et $\delta$. On appelle $\Pi_{p, q}$ (respectivement $\Pi_{q, s}, \ldots$ ) le plan contenant $p, q$ (respectivement $q, s, \ldots$ ) et $M$.

On commence par s'intéresser à l'intersection de $\Omega_{\infty}$ avec le plan $\Pi_{p, q}$ engendré par $p, q$ et $M$. On choisit une première courbe $\mathscr{C}_{p, q}$ qui joint $p$ à $q$ puis $q$ à $p$ et qui :

- est incluse dans la face de $D$ contenant $[p q]$, c'est-à-dire $\Pi_{p, q} \cap \bar{D}$;

- est strictement convexe et de classe $\mathscr{C}^{1}$;

- n'est pas de classe $\mathscr{C}^{1+\varepsilon}$ en $p$ et $q$ pour aucun $\varepsilon>0$.

On peut remarquer au passage que cette courbe est, par la dernière propriété, incluse dans $\mathbb{U}^{3}$ au voisinage de $p$ et $q$. On utilise à présent les symétries du groupe $\Gamma$ (c'est-à-dire celles de $P$ ) pour copier cette courbe et obtenir des courbes $\mathscr{C}_{p, s}, \mathscr{C}_{r, q}$ et $\mathscr{C}_{s, r}$ joignant $\{p, s\},\{r, q\}$ et $\{s, r\}$.
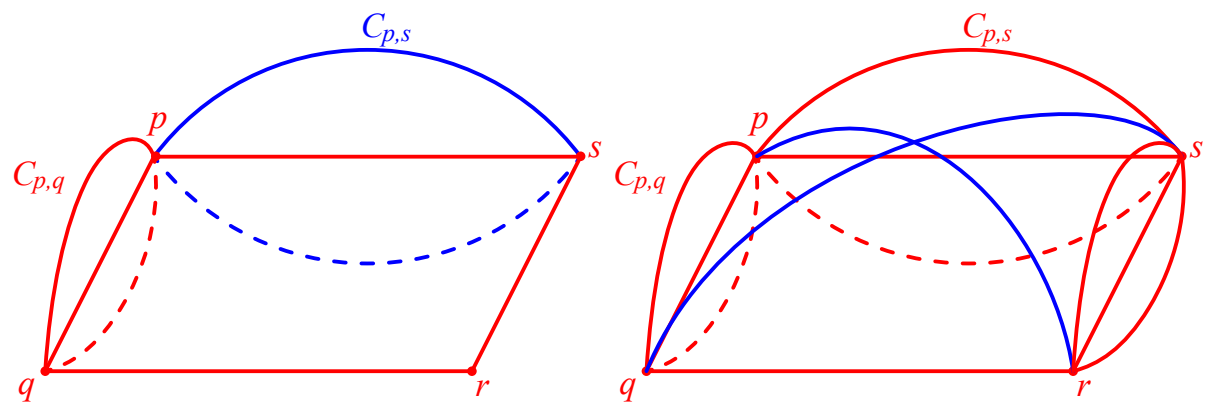

Figure 10. Les courbes et le dôme. 
Nous allons "relier" ces courbes pour construire le bord de $D_{1}$ (qu'on appellera le dôme). Pour simplifier la discussion, il est bon de remarquer que cet ensemble de courbes admet le même groupe de symétrie que le carré idéal de départ, à savoir un groupe diédral d'ordre 8 .

Soit $\gamma^{\mathbb{R}}$ (respectivement $\delta^{\mathbb{R}}$ ) le groupe à 1-paramètre engendré par $\gamma$ (respectivement $\delta$ ). Les orbites d'un point de $\Omega_{\infty} \backslash \Pi$ sous $\gamma^{\mathbb{R}}$ sont des demi-ellipses d'extrémités $\gamma^{-}$et $\gamma^{+}$; de même pour $\delta^{\mathbb{R}}$.

Le domaine fondamental $D$ privé de $\Pi_{p, r} \cup \Pi_{q, s}$ possède 4 composantes connexes $D_{\delta^{-}}, D_{\gamma^{-}}, D_{\delta^{+}}$et $D_{\gamma^{+}}$, naturellement étiquetées par $\delta^{-}, \gamma^{-}, \delta^{+}, \gamma^{+}$. L'orbite de $\mathscr{C}_{p, q}$ sous $\gamma^{\mathbb{R}}$ est une surface convexe ${ }^{2} S_{p, q}$, qui contient $\mathscr{C}_{s, r}$. De même, en considérant l'orbite de $\mathscr{C}_{r, q}$ sous $\delta^{\mathbb{R}}$, on obtient une surface convexe $S_{r, q}$. Soit $S$ la surface obtenue comme la réunion

$$
S=\left(D_{\delta^{-}} \cap S_{r, q}\right) \cup\left(D_{\gamma^{-}} \cap S_{p, q}\right) \cup\left(D_{\delta^{+}} \cap S_{r, q}\right) \cup\left(D_{\gamma^{+}} \cap S_{p, q}\right) .
$$

La surface $S$ possède encore un groupe diédral d'ordre 8 de symétries.

Appelons $D_{0}$ l'adhérence dans $D$ de l'enveloppe convexe de la surface $S$. L'ensemble $D_{0}$ est convexe, inclus dans $\mathbb{M}^{3}$. La réunion $\Omega_{0}=\bigcup_{\gamma \in \Gamma} \gamma\left(D_{0}\right)$ est un ouvert proprement convexe. En effet, $\gamma\left(D_{0}\right) \cup D_{0}$ est encore convexe puisque, par construction, les surfaces $S_{p, q}$ et $\gamma\left(S_{p, q}\right)$ se recollent pour donner une surface convexe ; bien sûr, le même chose est valable pour $\delta$; le résultat pour $\Gamma$ s'en déduit à l'aide d'une récurrence sur la longueur d'un élément pour la métrique des mots de $\Gamma$.

L'ouvert proprement convexe $\Omega_{0}$ est strictement convexe mais son bord n'est pas de classe $\mathscr{C}^{1}$ a priori. En dehors des courbes $\mathscr{C}_{p, r}=\Pi_{p, r} \cup \partial \Omega_{0}$ et $\mathscr{C}_{q, s}=\Pi_{q, s} \cup \partial \Omega_{0}$ et de leurs images par $\Gamma$, la surface $\partial \Omega_{0}$ est de classe $\mathscr{C}^{1}$. En lissant $\Omega_{0}$ le long de ces courbes, on obtient qu'il existe un voisinage $\mathscr{V}$ de $\mathscr{C}_{p, r} \cup \mathscr{C}_{q, s}$ dans $\partial \Omega_{0}$ et un convexe $D_{1}$ tel que $\partial D_{0} \backslash \mathscr{V}=\partial D_{1} \backslash \mathscr{V}$. L'ensemble $\Omega_{1}=\bigcup_{\gamma \in \Gamma} \gamma\left(D_{1}\right)$ est alors un ouvert proprement convexe, strictement convexe, à bord $\mathscr{C}^{1}$ et $\Gamma$-invariant, mais son bord n'est pas de classe $\mathscr{C}^{1+\varepsilon}$ aux points paraboliques de $\Lambda_{\Gamma}$, pour tout $\varepsilon>0$.

8B. Représentation sphérique de $\mathrm{SL}_{2}(\mathbb{R})$ dans $\mathrm{SL}_{5}(\mathbb{R})$. Dans [Crampon et Marquis 2012], on avait introduit deux notions de finitude géométrique, la finitude géométrique sur $\Omega$ et sur $\partial \Omega$. Dans l'article présent, nous n'avons étudié que la première.

Comme exemple d'action géométriquement finie sur $\partial \Omega$ mais pas sur $\Omega$, on avait donné la représentation sphérique de $\mathrm{SL}_{2}(\mathbb{R})$ dans $\mathrm{SL}_{5}(\mathbb{R})$ : il s'agit de l'action de $\mathrm{SL}_{2}(\mathbb{R})$ sur l'espace $V_{4}=\mathbb{R}_{4}[X, Y]$ des polynômes homogènes de degré 4 en deux variables, sur lequel $\mathrm{SL}_{2}(\mathbb{R})$ agit par coordonnées. Notons $G<\mathrm{SL}_{5}(\mathbb{R})$ l'image de $\mathrm{SL}_{2}(\mathbb{R})$ par cette représentation.

2. On dit qu'une hypersurface de $\mathbb{P}^{n}$ est convexe si elle est une partie du bord d'un convexe de $\mathbb{P}^{n}$. 
Rappelons ce qui a été vu dans [Crampon et Marquis 2012]. Il existe un point $x \in \mathbb{P}^{4}$ dont l'orbite sous $G$ est la courbe de Veronese, dont une équation est donnée par :

$$
[t: s] \in \mathbb{P}^{1} \rightarrow\left[t^{4}: t^{3} s: t^{2} s^{2}: t s^{3}: s^{4}\right] \in \mathbb{P}^{4}
$$

L'ensemble des ouverts proprement convexes préservés par cette représentation de $\mathrm{SL}_{2}(\mathbb{R})$ dans $\mathrm{SL}_{5}(\mathbb{R})$ forme une famille croissante $\left\{\Omega_{r}, 0 \leqslant r \leqslant \infty\right\}$. L'ouvert convexe $\Omega_{0}$ est l'enveloppe convexe de la courbe de Veronese, et $\Omega_{\infty}$ est son dual; ces deux convexes ne sont ni strictement convexes ni à bord $\mathscr{C}^{1}$. Les ouverts convexes $\Omega_{r}, 0<r<\infty$ sont les $r$-voisinages de $\Omega_{0}$ dans la géométrie de Hilbert $\left(\Omega_{\infty}, d_{\Omega_{\infty}}\right)$. On a vu dans [Crampon et Marquis 2012] que ces convexes étaient strictement convexes et à bord $\mathscr{C}^{1}$. En fait, on peut voir en procédant comme dans la section 7 que :

Proposition 8.2. Pour $0<r<\infty$, le bord de l'ouvert convexe $\Omega_{r}$ est de classe $\mathscr{C}^{4 / 3}$ et 4-convexe.

Démonstration. L'ensemble limite $\Lambda_{G}$ de l'action de $G$ sur $\Omega_{r}$ est dans tous les cas la courbe de Veronese. Hors de l'ensemble limite, le bord de $\Omega_{r}$ est lisse car l'action de $G$ sur $\partial \Omega_{r} \backslash \Lambda_{G}$ est libre et transitive (voir [Crampon et Marquis 2012, section 10]); $\partial \Omega_{r} \backslash \Lambda_{G}$ s'identifie donc à une orbite de $G$. De plus, le dual de $\Omega_{r}$ est un certain $\Omega_{r^{\prime}}$. Le fait que $\partial \Omega_{r^{\prime}} \backslash \Lambda_{G}$ soit lisse implique que $\partial \Omega_{r} \backslash \Lambda_{G}$ est 2-convexe. Autrement dit, le bord de $\Omega_{r}$ est lisse à hessien défini positif hors de l'ensemble limite.

La courbe de Veronese $\Lambda_{G}$ est une courbe algébrique lisse sur laquelle $G$ agit transitivement. La régularité $\partial \Omega_{r}$ est donc la même en tout point de $\Lambda_{G}$. Or, un point $x$ de $\Lambda_{G}$ est un point fixe d'un certain élément hyperbolique $g \in G$. La régularité de $\partial \Omega_{r}$ en $x$ se lit sur les valeurs propres de $g$. Il n'est pas dur de voir que si $g$ est l'image par la représentation d'un élément hyperbolique $\gamma$ de $\mathrm{SL}_{2}(\mathbb{R})$. Si $\lambda, \lambda^{-1}$ sont les valeurs propres de $\gamma$, avec $\lambda>1$, alors celles de $g$ sont $\lambda^{4}, \lambda^{2}, 1, \lambda^{-2}, \lambda^{-4}$. D'après la proposition 7.2 et le lemme 7.6, $\partial \Omega_{r}$ est $\mathscr{C}^{1+\varepsilon}$ en $x$ pour tout $\varepsilon<\varepsilon(g)$ avec

$$
\varepsilon(g)=\frac{\ln \lambda^{-2}-\ln \lambda^{-4}}{\ln \lambda^{4}-\ln \lambda^{-2}}=\frac{1}{3} .
$$

En fait, dans le cas d'un point fixe hyperbolique, on peut être plus précis dans la proposition 7.2 et voir que la valeur $\varepsilon(g)$ est atteinte, autrement dit que le bord est $\mathscr{C}^{1+\varepsilon(g)}$ en $x$ (et pas plus). On obtient donc que $\partial \Omega_{r}$ est $\mathscr{C}^{4 / 3}$ et, par dualité, que $\partial \Omega_{r}$ est 4-convexe. (En effet, le bord $\partial \Omega$ est $\beta$-convexe au point $x$ si et seulement si le bord $\partial \Omega^{*}$ du convexe dual est $\mathscr{C}^{1+\varepsilon}$ au point $x^{*}$, avec $1 /(1+\varepsilon)+1 / \beta=1$.) 


\section{Entropie volumique et exposant critique}

Si $\Gamma$ est un sous-groupe discret de $\operatorname{Aut}(\Omega)$, on notera, pour $x \in \Omega$ et $R \geqslant 0$,

$$
N_{\Gamma}(x, R)=\sharp\left\{g \in \Gamma \mid d_{\Omega}(x, g x) \leqslant R\right\}
$$

le nombre d'éléments $g$ de $\Gamma$ tels que $g x \in B(x, R)$. L'exposant critique du groupe $\Gamma$, défini par

$$
\delta_{\Gamma}=\limsup _{R \rightarrow+\infty} \frac{1}{R} \ln N_{\Gamma}(x, R),
$$

mesure le taux de croissance exponentiel du groupe $\Gamma$ agissant sur $\Omega$; il est immédiat que la limite précédente ne dépend pas du point $x$ considéré.

L'exposant critique $\delta_{\Gamma}$ de $\Gamma$ est nommé ainsi car c'est l'exposant critique des séries de Poincaré de $\Gamma$ données par

$$
g_{\Gamma}(s, x)=\sum_{\gamma \in \Gamma} e^{-s d_{\Omega}(x, \gamma x)}, \quad x \in \Omega
$$

cela veut dire que pour $s>\delta_{\Gamma}$, la série converge, et pour $s<\delta_{\Gamma}$, elle diverge.

L'entropie volumique d'une géométrie de Hilbert

$$
h_{\mathrm{vol}}(\Omega)=\limsup _{R \rightarrow+\infty} \frac{1}{R} \ln \operatorname{Vol}_{\Omega} B(x, R)
$$

représente le taux de croissance exponentiel du volume des boules de l'espace métrique $\left(\Omega, d_{\Omega}\right)$.

9A. Groupes de covolume fini. Si la géométrie $\left(\Omega, d_{\Omega}\right)$ admet une action cocompacte d'un sous-groupe discret $\Gamma$ de $\operatorname{Aut}(\Omega)$, on a évidemment l'égalité

$$
\delta_{\Gamma}=h_{\mathrm{vol}}(\Omega)
$$

puisqu'alors l'entropie volumique ne dépend pas de la mesure de volume considérée, pourvu qu'elle soit $\Gamma$-invariante; aussi peut-on prendre la mesure de comptage de l'orbite d'un point $x$ de $\Omega$ sous $\Gamma$ pour retrouver $\delta_{\Gamma}$. Si le groupe est "trop petit", cette égalité devient en général fausse, et on a seulement $\delta_{\Gamma} \leqslant h_{\mathrm{vol}}$. Dans [Dal'Bo et al. 2009], Françoise Dal'bo, Marc Peigné, Jean-Claude Picaud et Andrea Sambusetti ont étudié cette question pour les sous-groupes de covolume fini de variétés de Hadamard, à courbure négative pincée. Ils ont montré le résultat suivant.

Théorème 9.1. - Soit $M$ une variété riemannienne à courbure strictement négative, de volume fini. Si M est asymptotiquement $\frac{1}{4}$-pincée, alors $h_{\mathrm{vol}}=h_{\mathrm{top}}$.

- Pour tout $\varepsilon>0$, il existe une variété riemannienne de volume fini et de courbure strictement négative $\left(\frac{1}{4}+\varepsilon\right)$-pincée telle que $h_{\mathrm{top}}<h_{\mathrm{vol}}$. 
L'hypothèse de pincement asymptotique concerne la géométrie de la variété à l'infini, c'est-à-dire dans ses pointes. Dans notre contexte, c'est le corollaire 4.2 qui va nous permettre de montrer le prochain résultat :

Théorème 9.2. Soit $\Gamma$ un sous-groupe discret de $\operatorname{Aut}(\Omega)$, de covolume fini. Alors

$$
\delta_{\Gamma}=h_{\mathrm{vol}}(\Omega) .
$$

La démonstration de ce résultat est fort similaire à celle de [Dal'Bo et al. 2009], elle se simplifie par certains aspects et nécessite des arguments un peu différents par d'autres. Elle reste malgré tout un brin technique...

On va commencer par calculer l'exposant critique d'un sous-groupe parabolique de rang maximal.

Lemme 9.3. Soit $\Gamma$ un sous-groupe de $\operatorname{Aut}(\Omega)$ et $\operatorname{Aut}\left(\Omega^{\prime}\right)$ avec $\Omega \subset \Omega^{\prime}$. Appelons $g_{\Gamma, \Omega}(s, x)$ et $g_{\Gamma, \Omega^{\prime}}(s, x)$ les séries de Poincaré pour l'action de $\Gamma$ sur $\Omega$ et $\Omega^{\prime}$, $\delta_{\Gamma}(\Omega)$ et $\delta_{\Gamma}\left(\Omega^{\prime}\right)$ leur exposant critique. Alors, pour tout $s>\delta_{\Gamma}\left(\Omega^{\prime}\right)$,

$$
g_{\Gamma, \Omega}(s, x) \leqslant g_{\Gamma, \Omega^{\prime}}(s, x) .
$$

En particulier, $\delta_{\Gamma}(\Omega) \leqslant \delta_{\Gamma}\left(\Omega^{\prime}\right)$.

Démonstration. Pour $x, y \in \Omega$, on a $d_{\Omega^{\prime}}(x, y) \leqslant d_{\Omega}(x, y)$. Donc si $x \in \Omega$ et $s>\delta_{\Gamma}\left(\Omega^{\prime}\right)$, on a $g_{\Gamma, \Omega}(s, x) \leqslant g_{\Gamma, \Omega^{\prime}}(s, x)$. En particulier, la convergence de $g_{\Gamma, \Omega^{\prime}}(s, x)$ implique celle de $g_{\Gamma, \Omega}(s, x)$, d'où le résultat.

Lemme 9.4. L'exposant critique d'un sous-groupe parabolique de rang maximal $\mathscr{P}$ de $\operatorname{Aut}(\Omega)$ est $\delta_{\mathscr{P}}=(n-1) / 2$ et les séries de Poincaré de $\mathscr{P}$ divergent en $\delta_{\mathscr{P}}$ :

$$
\sum_{\gamma \in \mathscr{P}} e^{-\delta \Re d_{\Omega}(x, \gamma x)}=+\infty \quad \text { pour tout } x \in \Omega .
$$

Démonstration. Appelons $p$ le point fixe de $\mathscr{P}$. D'après le théorème 4.1 , on peut trouver deux ellipsoïdes $\mathscr{E}^{\text {int }}$ et $\mathscr{E}$ ext $\mathscr{P}$-invariants tels que

$$
\partial_{\mathscr{E}}^{\mathscr{E}^{\text {int }}} \cap \partial^{\mathscr{E}} \mathscr{E}^{\text {ext }}=\partial^{\mathscr{E}} \mathscr{E}^{\text {int }} \cap \partial \Omega=\partial^{\mathscr{E}}{ }^{\text {ext }} \cap \partial \Omega=\{p\} \quad \text { et } \quad \mathscr{E}^{\text {int }} \subset \Omega \subset \mathscr{E}^{\text {ext }} .
$$

Il est connu en géométrie hyperbolique que $\delta_{\mathscr{P}}\left(\mathscr{C}^{\mathrm{int}}\right)=\delta_{\mathscr{P}}\left(\mathscr{E}^{\mathrm{ext}}\right)=(n-1) / 2$ et que les séries de Poincaré de $\mathscr{P}$ divergent en l'exposant critique (on pourra consulter, par exemple, [Dal'bo et al. 2000, partie 3], même si les calculs, élémentaires, remontent à Alan Beardon, dans les années 1970). D'après le lemme 9.3, on a de même pour $\mathscr{P}$ agissant sur $\Omega$.

Nous aurons aussi besoin du lemme suivant :

Lemme 9.5. Soit $C>1$ arbitrairement proche de 1 et $\mathscr{P}$ un sous-groupe parabolique maximal de $\operatorname{Aut}(\Omega)$ fixant $p \in \partial \Omega$. Alors il existe une horoboule $H_{C}$ basée en $p$, 
d'horosphère au bord $\mathscr{H}_{C}$ et une constante $D>1$ telles que

$$
\frac{1}{D} N_{\mathscr{P}}\left(x, \frac{R}{C}\right) \leqslant \operatorname{Vol}_{\Omega}\left(B(x, R) \cap H_{C}\right) \leqslant D N_{\mathscr{P}}(x, C R), \quad x \in \mathscr{H}_{C}, R>0 .
$$

Démonstration. Dans l'espace hyperbolique, on sait (voir, par exemple, [Dal'Bo et al. 2009, proposition 3.3]) que, pour tout sous-groupe parabolique maximal $\mathscr{P}$, toute horoboule $H$ stable par $\mathscr{P}$, d'horosphère au bord $\mathscr{H}$, il existe un réel $D \geqslant 1$ tel que

(9-1) $\frac{1}{D} \operatorname{Vol}_{\mathbb{\sharp}^{n}}\left(B(x, R) \cap H_{C}\right) \leqslant N_{\mathscr{P}}(x, R) \leqslant D \operatorname{Vol}_{\mathbb{\sharp}^{n}}(B(x, R) \cap H), \quad x \in \mathscr{H}$.

Soit donc $\mathscr{P}$ un sous-groupe parabolique maximal de $\operatorname{Aut}(\Omega)$ fixant $p \in \partial \Omega$. Le corollaire 4.2 nous fournit une horoboule $H_{C}$ basée en $p$ qui porte deux métriques

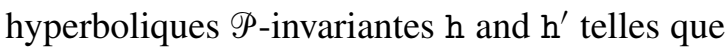

$$
\frac{1}{C} \mathrm{~h}^{\prime} \leqslant \mathrm{h} \leqslant F \leqslant \mathrm{~h}^{\prime} \leqslant C \mathrm{~h} .
$$

Prenons $x \in \mathscr{H}$. D'après la proposition 2.1, on a, pour tout $R>0$,

$$
B_{\mathrm{h}^{\prime}}\left(x, \frac{R}{C}\right) \subset B_{\mathrm{h}}(x, R) \subset B(x, R) \subset B_{\mathrm{h}^{\prime}}(x, R) \subset B_{\mathrm{h}}(x, C R),
$$

où, par $B_{\mathrm{h}}$ et $B_{\mathrm{h}^{\prime}}$, on note les boules métriques pour h et $\mathrm{h}^{\prime}$. En appelant $\mathrm{Vol}_{\mathrm{h}}$ et $\mathrm{Vol}_{\mathrm{h}^{\prime}}$ les volumes riemanniens associés à $\mathrm{h}$ et $\mathrm{h}^{\prime}$, on a, toujours d'après la proposition 2.1,

$$
\operatorname{Vol}_{h^{\prime}} \leqslant \operatorname{Vol}_{\Omega} \leqslant \operatorname{Vol}_{\mathrm{h}}
$$

Ainsi,

$$
\operatorname{Vol}_{\mathrm{h}^{\prime}}\left(B_{\mathrm{h}^{\prime}}\left(x, \frac{R}{C}\right) \cap H\right) \leqslant \operatorname{Vol}_{\Omega}(B(x, R) \cap H) \leqslant \operatorname{Vol}_{\mathrm{h}}\left(B_{\mathrm{h}}(x, C R) \cap H\right) .
$$

D'après l'encadrement (9-1), il existe une constante $D>1$ telle que

$$
\frac{1}{D} N_{\mathscr{P}}^{\mathrm{h}^{\prime}}\left(x, \frac{R}{C}\right) \leqslant \operatorname{Vol}_{\Omega}(B(x, R) \cap H) \leqslant D N_{\mathscr{P}}^{\mathrm{h}}(x, C R),
$$

où $N_{\mathscr{P}}^{\mathrm{h}}(x, R)$ est le nombre de points de l'orbite $\mathscr{P} \cdot x$ dans la boule de rayon $R$ pour $\mathrm{h}$; de même pour $\mathrm{h}^{\prime}$.

(Bien entendu, les horoboules considérées dans l'encadrement (9-1) sont les horoboules hyperboliques et pas celles de $F$, et il faut donc faire un peu plus attention lorsqu'on dit qu'une telle constante $D$ existe. Si $\mathscr{H}_{\mathrm{h}}$ est l'horosphère pour h basée en $p$ et passant par $x$, alors la h-distance maximale entre $\mathscr{H}$ et $\mathscr{H}_{\mathrm{h}}$ est finie, car $\mathscr{P}$ agit de façon cocompacte sur $\mathscr{H} \backslash\{p\}$ et $\mathscr{H}_{\mathrm{h}} \backslash\{p\}$. Donc, pour une certaine constante $D^{\prime}>0$, on a, pour tout $R>0$,

$$
\left|\operatorname{Vol}_{\mathrm{h}}\left(B_{\mathrm{h}}(x, R) \cap H\right)-\operatorname{Vol}_{\mathrm{h}}\left(B_{\mathrm{h}}(x, R) \cap H_{\mathrm{h}}\right)\right| \leqslant D^{\prime} N_{\mathscr{P}}(x, R),
$$

où $H_{\mathrm{h}}$ est l'horoboule définie par $\mathscr{H}_{\mathrm{h}}$. D'où l'existence de $D$.) 
Pour conclure, il suffit de remarquer que, comme $\mathrm{h} \leqslant F \leqslant \mathrm{~h}^{\prime}$, on a

$$
N_{\mathscr{P}}^{\mathrm{h}}(x, R) \leqslant N_{\mathscr{P}}(x, R) \leqslant N_{\mathscr{P}}^{\mathrm{h}^{\prime}}(x, C) .
$$

Démonstration du théorème 9.2. On sait déjà que $\delta_{\Gamma} \leqslant h_{\mathrm{vol}}$, et il faut donc seulement prouver l'inégalité inverse.

Fixons $C>1$ arbitrairement proche de 1, et choisissons $o \in \Omega$ ainsi qu'un domaine fondamental convexe localement fini pour l'action de $\Gamma$ sur $\Omega$, qui contienne le point $o$. Décomposons ce domaine fondamental en

$$
C_{0} \sqcup \bigsqcup_{1 \leqslant i \leqslant l} C_{i},
$$

où $C_{0}$ est compact et les $C_{i}, 1 \leqslant i \leqslant l$, correspondent aux pointes $\xi_{i} \in \partial \Omega$ : chaque $C_{i}$ est une partie d'un domaine fondamental pour l'action d'un sous-groupe parabolique maximal $\mathscr{P}_{i}$ sur une horoboule $H_{\xi_{i}}$ basée au point $\xi_{i}$; les points $\xi_{i}$ sont les points de $\partial \Omega$ adhérents au domaine fondamental. On suppose que les $H_{\xi_{i}}$ sont choisies de telle façon qu'elles satisfassent au lemme 9.5 pour la constante $C$ qu'on a fixée.

La boule $B(o, R)$ de rayon $R \geqslant 0$ peut être décomposée en

$$
B(o, R)=\left(\Gamma \cdot C_{0} \cap B(o, R)\right) \sqcup \bigsqcup_{1 \leqslant i \leqslant l}\left(\Gamma \cdot H_{\xi_{i}} \cap B(o, R)\right),
$$

de telle façon que

$$
\operatorname{Vol}_{\Omega}(B(o, R))=\operatorname{Vol}_{\Omega}\left(\Gamma \cdot C_{0} \cap B(o, R)\right)+\sum_{i=1}^{l} \operatorname{Vol}_{\Omega}\left(\Gamma \cdot H_{\xi_{i}} \cap B(o, R)\right) .
$$

Pour le premier terme, on a $\operatorname{Vol}_{\Omega}\left(\Gamma \cdot C_{0} \cap B(o, R)\right) \leqslant N_{\Gamma}(o, R) \operatorname{Vol}_{\Omega}\left(C_{0}\right)$; c'est donc le second qu'il nous faut étudier.

Pour chaque horoboule $H_{\gamma \xi_{i}}=\gamma H_{\xi_{i}}$, appelons $x_{\gamma, i}$ le point d'intersection de la droite $\left(o \gamma \xi_{i}\right)$ avec l'horosphère $\partial H_{\gamma \xi_{i}} \backslash\left\{\gamma \xi_{i}\right\}$, qui n'est rien d'autre que la projection de $o$ sur $H_{\gamma \xi_{i}}$. Pour chaque $\gamma \in \Gamma$, notons $\bar{\gamma} \in \Gamma$ un des éléments $g \in \Gamma$, en nombre fini, tels que $x_{\gamma, i} \in g \cdot C_{i} ; \bar{\gamma}$ est le "premier élément pour lequel $H_{\gamma \xi_{i}}$ intersecte $B(o, R)$." Appelons $\bar{\Gamma}$ l'ensemble de ces éléments $\bar{\gamma}$.

La remarque principale est le lemme ci-dessous, équivalent du fait classique suivant en courbure négative pincée : pour chaque $\theta \in(0, \pi)$, on peut trouver une constante $C(\theta)$ telle que, pour chaque triangle géodésique $x y z$ dont l'angle au point $y$ est au moins $\theta$, le chemin $x \rightarrow y \rightarrow z$ sur le triangle est une quasi-géodésique entre $x$ et $z$ avec une erreur au plus $C(\theta)$.

Lemme 9.6. Il existe $r>0$ tel que, pour chaque $\gamma \in \Gamma, 1 \leqslant i \leqslant l$ et $z \in H_{\gamma \xi_{i}}$, le chemin formé des segments $\left[o x_{\gamma, i}\right]$ et $\left[x_{\gamma, i} z\right]$ est une quasi-géodésique avec une erreur au plus $r$, c'est-à-dire que

$$
d_{\Omega}(o, z) \geqslant d_{\Omega}\left(o, x_{\gamma, i}\right)+d_{\Omega}\left(x_{\gamma, i}, z\right)-r .
$$


Démonstration. Prenons $\gamma \in \Gamma, 1 \leqslant i \leqslant l$ et $z \in H_{\gamma \xi_{i}}$. Rappelons que l'espace métrique $\left(\Omega, d_{\Omega}\right)$ est Gromov-hyperbolique (voir [Crampon et Marquis 2012, section 9]). Aussi existe t-il un réel $\delta \geqslant 0$ pour lequel chaque triangle géodésique est $\delta$-fin. Ainsi, il existe $p \in[o z]$ tel que

$$
d_{\Omega}\left(p,\left[x_{\gamma, i} z\right]\right) \leqslant \delta, \quad d_{\Omega}\left(p,\left[o x_{\gamma, i}\right]\right) \leqslant \delta .
$$

On peut donc trouver des points $o^{\prime} \in\left[o x_{\gamma, i}\right]$ et $z^{\prime} \in\left[x_{\gamma, i} z\right]$ de telle façon que

$$
d_{\Omega}\left(o^{\prime}, p\right)+d_{\Omega}\left(p, z^{\prime}\right) \leqslant 2 \delta .
$$

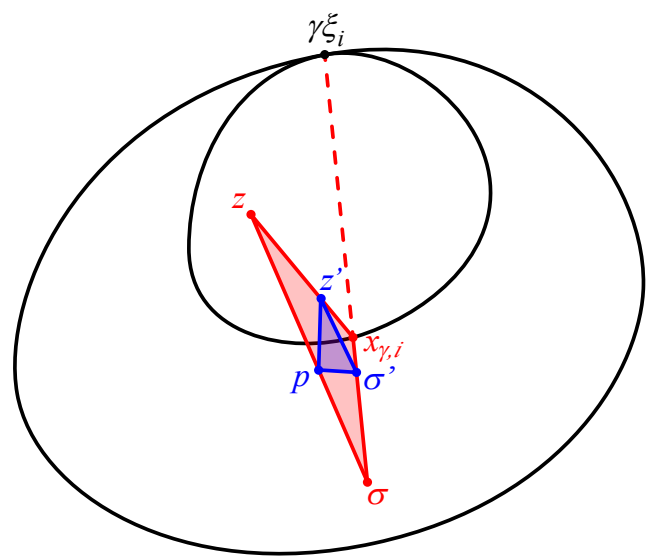

Par l'inégalité triangulaire, la distance entre $o^{\prime}$ et $z^{\prime}$ est alors plus petite que $2 \delta$. Puisque $x_{\gamma, i}$ est la projection de $o^{\prime}$ sur l'horoboule $H_{\gamma \xi_{i}}$ et que $z^{\prime}$ est dans l'horoboule $H_{\gamma \xi_{i}}$, on a $d_{\Omega}\left(o^{\prime}, x_{\gamma, i}\right) \leqslant d_{\Omega}\left(o^{\prime}, z^{\prime}\right) \leqslant 2 \delta$ et, par l'inégalité triangulaire, $d_{\Omega}\left(x_{\gamma, i}, z^{\prime}\right) \leqslant 4 \delta$. Ainsi, on obtient

$$
d_{\Omega}\left(o^{\prime}, x_{\gamma, i}\right)+d_{\Omega}\left(x_{\gamma, i}, z^{\prime}\right) \leqslant 6 \delta .
$$

Cela amène

$$
\begin{aligned}
d_{\Omega}\left(o, x_{\gamma, i}\right)+d_{\Omega}\left(x_{\gamma, i}, z\right) & \leqslant d_{\Omega}\left(o, o^{\prime}\right)+d_{\Omega}\left(o^{\prime}, x_{\gamma, i}\right)+d_{\Omega}\left(x_{\gamma, i}, z^{\prime}\right)+d_{\Omega}\left(z^{\prime}, z\right) \\
& \leqslant 6 \delta+d_{\Omega}(o, p)+d_{\Omega}\left(p, o^{\prime}\right)+d_{\Omega}\left(z^{\prime}, p\right)+d_{\Omega}(p, z) \\
& \leqslant 8 \delta+d_{\Omega}(o, z) .
\end{aligned}
$$

Maintenant, si $z$ est un point dans $\gamma \cdot H_{\xi_{i}} \cap B(o, R)$, pour certains $\gamma \in \Gamma, 1 \leqslant i \leqslant l$ et $R>0$, le lemme 9.6 implique que

$$
d_{\Omega}\left(o, x_{\gamma, i}\right)+d_{\Omega}\left(x_{\gamma, i}, z\right) \leqslant d_{\Omega}(o, z)+r \leqslant R+r .
$$

Or, il existe $c \geqslant 0$ tel que $d_{\Omega}\left(o, x_{\gamma, i}\right) \geqslant d_{\Omega}(o, \bar{\gamma} o)-c$ : il suffit de prendre pour $c$ la distance maximale entre $o$ et le bord $\partial C_{i} \cap \partial H_{\xi_{i}} \backslash\left\{\xi_{i}\right\}$. D'où

$$
d_{\Omega}\left(x_{\gamma, i}, z\right) \leqslant R+r-d_{\Omega}(o, \bar{\gamma} o)+c .
$$


Posons $K=r+c$. Pour tous $\gamma \in \Gamma, 1 \leqslant i \leqslant l$, et $R>0$, on a ainsi

$$
\gamma \cdot H_{\xi_{i}} \cap B(o, R) \subset \gamma \cdot H_{\xi_{i}} \cap B\left(x_{\gamma, i}, R-d_{\Omega}(o, \bar{\gamma} o)+K\right) .
$$

Cela permet d'évaluer le volume $\operatorname{Vol}_{\Omega}\left(\Gamma \cdot H_{\xi_{i}} \cap B(o, R)\right)$. En effet,

$$
\begin{aligned}
\operatorname{Vol}_{\Omega}\left(\Gamma \cdot H_{\xi_{i}} \cap B(o, R)\right) & =\sum_{\bar{\gamma} \in \bar{\Gamma}} \operatorname{Vol}_{\Omega}\left(\bar{\gamma} \cdot H_{\xi_{i}} \cap B(o, R)\right) \\
& \leqslant \sum_{\bar{\gamma} \in \bar{\Gamma}} \operatorname{Vol}_{\Omega}\left(\bar{\gamma} \cdot H_{\xi_{i}} \cap B\left(x_{\gamma, i}, R-d(o, \bar{\gamma} o)+K\right)\right) \\
& \leqslant \sum_{0 \leqslant k \leqslant[R]} \sum_{\substack{\bar{\gamma} \in \bar{\Gamma} \\
k \leqslant d_{\Omega}(o, \bar{\gamma} o) \leqslant k+1}} \operatorname{Vol}_{\Omega}\left(\bar{\gamma} \cdot H_{\xi_{i}} \cap B\left(x_{\gamma, i}, R-k+K\right)\right) \\
& \leqslant \sum_{0 \leqslant k \leqslant[R]} N_{\bar{\Gamma}}(o, k, k+1) \operatorname{Vol}_{\Omega}\left(H_{\xi_{i}} \cap B\left(x_{i}, R-k+K\right)\right),
\end{aligned}
$$

où $x_{i}=x_{\mathrm{Id}, i}$ et, pour toute partie $S$ de $\Gamma$ et tout $0 \leqslant r<R$,

$$
N_{S}(o, r, R)=\sharp\left\{\gamma \in S \mid r \leqslant d_{\Omega}(o, \gamma o)<R\right\} .
$$

Le lemme 9.5 donne

(9-2) $\operatorname{Vol}_{\Omega}\left(\Gamma \cdot H_{\xi_{i}} \cap B(o, R)\right) \leqslant D \sum_{0 \leqslant k \leqslant[R]} N_{\bar{\Gamma}}\left(x_{i}, k, k+1\right) N_{\mathscr{S}_{i}}\left(x_{i}, C(R-k+K)\right)$

pour une certaine constante $D>1$ qui peut être choisie indépendante de $i$. De plus, comme l'exposant critique de chaque $\mathscr{P}_{i}$ est $(n-1) / 2$, il existe un réel $M \geqslant 1$, indépendant de $i$ mais dépendant de $C$, tel que

$$
\left.\frac{1}{M} e^{\left(\frac{n-1}{2}-(C-1)\right) R} \leqslant N_{\mathscr{P}_{i}}\left(x_{i}, R\right)\right) \leqslant M e^{\left(\frac{n-1}{2}+(C-1)\right) R} .
$$

D’un côté, cela implique que

$$
N_{\mathscr{P}_{i}}\left(x_{i}, C(R-k+K)\right) \leqslant L N_{\mathscr{P}_{i}}\left(x_{i}, C(R-k)\right),
$$

où $L=M^{2} e^{\left(\frac{n-1}{2}-(C-1)\right) C K}$. D'un autre côté, cela nous donne

$$
\begin{aligned}
N_{\mathscr{P}_{i}}\left(x_{i}, C R\right) & \leqslant M e^{\left(\frac{n-1}{2}+(C-1)\right) C R} \\
& =M e^{\left(\frac{n-1}{2}-(C-1)\right) R} e^{\left(\frac{n-1}{2}+C+1\right)(C-1) R} \\
& \leqslant M^{2} e^{\left(\frac{n-1}{2}+C+1\right)(C-1) R} N_{\mathscr{P}_{i}}\left(x_{i}, R\right) .
\end{aligned}
$$

Avec (9-2), on obtient

(9-3) $\operatorname{Vol}_{\Omega}\left(\Gamma \cdot H_{\xi_{i}} \cap B(o, R)\right) \leqslant$

$$
D L M^{2} e^{\left(\frac{n-1}{2}+C+1\right)(C-1) R} \sum_{0 \leqslant k \leqslant[R]} N_{\bar{\Gamma}}\left(x_{i}, k, k+1\right) N_{\mathscr{P}_{i}}\left(x_{i}, R-k\right) .
$$


Finalement, remarquons que tout élément $\gamma \in \Gamma$ tel que $d_{\Omega}\left(x_{i}, \gamma x_{i}\right)<R$ peut être écrit de façon unique $\gamma=\bar{\gamma}_{i} p_{i}$, avec $d_{\Omega}\left(x_{i}, \bar{\gamma}_{i} x_{i}\right)<R$ et $p_{i} \in \mathscr{P}_{i}$, de telle façon que

$$
d_{\Omega}\left(x_{i}, p_{i} x_{i}\right)+d_{\Omega}\left(x_{i}, \bar{\gamma}_{i} x_{i}\right) \geqslant R \text {. }
$$

D'où

$$
N_{\Gamma}\left(x_{i}, R\right) \geqslant \sum_{0 \leqslant k \leqslant[R]} N_{\bar{\Gamma}}\left(x_{i}, k, k+1\right) N_{\mathscr{P}_{i}}\left(x_{i}, R-k\right) .
$$

Les inégalités (9-3) et (9-4) impliquent alors

$$
\operatorname{Vol}_{\Omega}\left(\Gamma \cdot H_{\xi_{i}} \cap B(o, R)\right) \leqslant D L M^{2} e^{\left(\frac{n-1}{2}+C+1\right)(C-1) R} N_{\Gamma}\left(x_{i}, R\right) ;
$$

et donc, en mettant tout ensemble

$$
\operatorname{Vol}_{\Omega}(B(o, R)) \leqslant N e^{\left(\frac{n-1}{2}+C+1\right)(C-1) R} N_{\Gamma}(o, R),
$$

pour un certain réel $N>1$. Cela donne

$$
h_{\mathrm{vol}} \leqslant \delta_{\Gamma}+\left(\frac{n-1}{2}+C+1\right)(C-1) .
$$

Comme $C$ est arbitrairement proche de 1 , on obtient $h_{\mathrm{vol}} \leqslant \delta_{\Gamma}$.

9B. Groupes dont l'action est géométriquement finie sur $\Omega$. En fait, par la même démonstration et les résultats de [Crampon et Marquis 2012], on peut obtenir un résultat similaire pour des groupes dont l'action est géométriquement finie sur $\Omega$ : Théorème 9.7. Soit $\Gamma$ un sous-groupe discret de $\operatorname{Aut}(\Omega)$ dont l'action sur $\Omega$ est géométriquement finie. Alors

$$
\delta_{\Gamma}=\limsup _{R \rightarrow+\infty} \frac{1}{R} \ln \operatorname{Vol}_{\Omega}\left(B(o, R) \cap C\left(\Lambda_{\Gamma}\right)\right),
$$

où o est un point quelconque de $\Omega$.

Nous avons préféré présenter la démonstration dans le cas du volume fini que nous considérions déjà assez technique pour ne pas la surcharger. Les seuls points à vérifier pour étendre le résultat sont les trois lemmes 9.4, 9.5 et 9.6 ; le reste se lit tel quel en pensant seulement à considérer l'intersection avec $C\left(\Lambda_{\Gamma}\right)$.

Pour le lemme 9.6, il suffit de se souvenir que, d'après [Crampon et Marquis 2012], l'espace $\left(C\left(\Lambda_{\Gamma}\right), d_{\Omega}\right)$ est Gromov-hyperbolique.

Pour les deux autres, il nous faut rappeler quelques éléments de [Crampon et Marquis 2012], dont on conseille de consulter la partie 7.

Tous les groupes paraboliques apparaissant dans une action géométriquement finie d'un groupe $\Gamma$ sur $\Omega$ sont conjugués dans $\mathrm{SL}_{n+1}(\mathbb{R})$ à des sous-groupes paraboliques de $\mathrm{SO}_{n, 1}(\mathbb{R})$. En particulier, un sous-groupe parabolique $\mathscr{P}$ de $\Gamma$ est 
virtuellement isomorphe à $\mathbb{Z}^{d}$ pour un certain $1 \leqslant d \leqslant n-1 ; d$ est le rang de $\mathscr{P}$. De plus, si $p \in \partial \Omega$ est le point fixe de $\mathscr{P}$, il existe une coupe $\Omega_{p}$ de dimension $d+1$ de $\Omega$, contenant $p$ dans son adhérence, c'est-à-dire l'intersection de $\Omega$ avec un sous-espace projectif de dimension $d+1$, qui est préservée par $\mathscr{P}$; ainsi, $\mathscr{P}$ apparaît comme un sous-groupe parabolique de rang maximal de $\operatorname{Aut}\left(\Omega_{p}\right)$. On obtient la généralisation suivante du lemme 9.4 :

Lemme 9.8. L'exposant critique d'un sous-groupe parabolique $\mathscr{P}$ de $\operatorname{Aut}(\Omega)$, de rang $d \leqslant n-1$, est $\delta_{\mathscr{P}}=d / 2$ et les séries de Poincaré de $\mathscr{P}$ divergent en $\delta_{\mathscr{P}}$ :

$$
\sum_{\gamma \in \mathscr{P}} e^{-\delta \Re d_{\Omega}(x, \gamma x)}=+\infty \quad \text { pour tout } x \in \Omega .
$$

Démonstration. Comme rien ne dépend pas du point base considéré, on peut le prendre dans le convexe $\Omega_{p}$ pour se ramener au cas original du lemme 9.4.

Le lemme 9.5 s'étendrait immédiatement sous les conclusions du corollaire 4.2. En général, on peut étendre au moins la majoration, qui est le point que l'on utilise dans la démonstration du théorème :

Lemme 9.9. Soit $C>1$ arbitrairement proche de 1 et $\mathscr{P}$ un sous-groupe parabolique maximal de $\operatorname{Aut}(\Omega)$ fixant $p \in \partial \Omega$. Il existe une horoboule $H_{C}$ basée en $p$ d'horosphère au bord $\mathscr{H}_{C}$ et une constante $D>1$ telle que

$$
\operatorname{Vol}_{\Omega}\left(B(x, R) \cap H \cap C\left(\Lambda_{\Gamma}\right)\right) \leqslant D N_{\mathscr{P}}(x, C R), \quad x \in \mathscr{H}_{C} \cap C\left(\Lambda_{\Gamma}\right), R \geqslant 1 .
$$

On aura besoin du lemme 9.12 ci-dessous. Il se déduit du fait suivant :

Lemme 9.10 [Colbois et Vernicos 2006]. Pour tout $m \geq 1$ et $R>0$, il existe deux constantes $v_{m}(R), V_{m}(R)>0$ telles que, pour tout ouvert proprement convexe $\Omega$ de $\mathbb{P}^{m}$ et $x \in \Omega$,

$$
v_{m}(R) \leqslant \operatorname{Vol}_{\Omega}(B(x, R)) \leqslant V_{m}(R) .
$$

Remarque 9.11. Le lemme précédent est contenu dans [Colbois et Vernicos 2006, théorème 12], qui donne en plus des bornes explicites, dont la dépendance en $R$ est exponentielle. Pour l'énoncé présenté ici, on peut donner une démonstration qualitative, basé sur le théorème de compacité de Benzécri [1960]; ce théorème affirme que l'action de $\mathrm{SL}_{n+1}(\mathbb{R})$ sur l'ensemble des couples $(\Omega, x)$, où $\Omega$ est un ouvert proprement convexe de $\mathbb{P}^{n}$ et $x$ un point de $\Omega$, est propre et cocompacte. On peut trouver cette démonstration dans [Crampon et Marquis 2012].

Lemme 9.12. Soient $r>0$ et $1 \leqslant d \leqslant n$. Il existe deux constantes $M, m>0$, dépendant seulement de $r, n$ et $d$, telles que, pour tout ouvert proprement convexe $\Omega$ de $\mathbb{P}^{n}$, tout sous-espace $\mathbb{P}^{d}$ de dimension d intersectant $\Omega$ et toute partie A compacte 
de l'ouvert proprement convexe $\Omega_{d}=\mathbb{P}^{d} \cap \Omega$ de $\mathbb{P}^{d}$, le volume du $r$-voisinage $V_{r}(A)$ de A dans $\Omega$ est comparable au volume du $r$-voisinage de A dans $\Omega_{d}$ :

$$
m \leqslant \frac{\operatorname{Vol}_{\Omega}\left(V_{r}(A)\right)}{\operatorname{Vol}_{\Omega_{d}}\left(V_{r}(A) \cap \Omega_{d}\right)} \leqslant M .
$$

Démonstration. Considérons un ensemble $\left\{x_{i}\right\}_{1 \leqslant i \leqslant N} 2 r$-séparé maximal de $A$ : deux points $x_{i}, x_{j}, i \neq j$, sont à distance au moins $2 r$ et il est impossible de rajouter un point à l'ensemble qui satisfasse à cette propriété. En particulier, les boules de rayon $r$ centrées aux points $x_{i}$ sont disjointes et incluses dans $V_{r}(A)$, alors que $V_{r}(A)$ est recouvert par les boules de rayon $4 r$ centrées aux points $x_{i}$. On a ainsi, en utilisant le lemme 9.10,

$$
\begin{aligned}
N v_{n}(r) & \leqslant \sum_{i=1}^{N} \operatorname{Vol}_{\Omega}\left(B\left(x_{i}, r\right)\right) \leqslant \operatorname{Vol}_{\Omega}\left(V_{r}(A)\right) \\
& \leqslant \sum_{i=1}^{N} \operatorname{Vol}_{\Omega}\left(B\left(x_{i}, 4 r\right)\right) \leqslant N V_{n}(4 r) ;
\end{aligned}
$$

de même,

$$
N v_{d}(r) \leqslant \operatorname{Vol}_{\Omega_{d}}\left(V_{r}(A) \cap \Omega_{d}\right) \leqslant N V_{d}(4 r) .
$$

En prenant le quotient, on obtient

$$
m:=\frac{v_{n}(r)}{V_{d}(4 r)} \leqslant \frac{\operatorname{Vol}_{\Omega}\left(V_{r}(A)\right)}{\operatorname{Vol}_{\Omega_{d}}\left(V_{r}(A) \cap \Omega_{d}\right)} \leqslant \frac{V_{n}(4 r)}{v_{d}(r)}=: M .
$$

Démonstration du lemme 9.9. Notons $d$ le rang de $\mathscr{P}$ et $\Omega_{p}=\Omega \cap \mathbb{P}^{d+1}$ une coupe de $\Omega$ de dimension $d+1$, contenant $p$ dans son adhérence, et préservée par $\mathscr{P}$. Pour toute horoboule $H$ basée en $p$, l'intersection $C\left(\Lambda_{\Gamma}\right) \cap H$ est dans un $d_{\Omega}$-voisinage de taille $r=r(H) \geqslant 0$ finie de $\Omega_{p} \cap C\left(\Lambda_{\Gamma}\right)$. Cela est simplement dû au fait que $\mathscr{P}$ agit de façon cocompacte sur $C\left(\Lambda_{\Gamma}\right) \cap \mathscr{H}$, où $\mathscr{H}$ est l'horosphère au bord de $H$. De plus, comme le bord de $\Omega$ est $\mathscr{C}^{1}$ en $p$, on peut, en considérant une horoboule plus petite, prendre $r$ aussi petit que l'on veut.

On fixe l'horoboule $H$ de telle façon que l'intersection $H \cap \Omega_{p}$, qui est une horoboule de $\Omega_{p}$ basée en $p$, satisfasse au lemme 9.5 pour $\Omega_{p}$, avec la constante $C$. On fixe aussi $r>0$ tel que $C\left(\Lambda_{\Gamma}\right) \cap H$ est dans un $d_{\Omega}$-voisinage de taille $r$ de $\Omega_{p} \cap C\left(\Lambda_{\Gamma}\right)$.

Si $x$ est un point de $\mathscr{H} \cap C\left(\Lambda_{\Gamma}\right)$, il existe un point $x^{\prime}$ de $\mathscr{H} \cap \Omega_{p}$ à distance moins que $r$ de $x$; on a alors $B(x, R) \subset B\left(x^{\prime}, R+r\right)$ et

$$
\operatorname{Vol}_{\Omega}\left(B(x, R) \cap H \cap C\left(\Lambda_{\Gamma}\right)\right) \leqslant \operatorname{Vol}_{\Omega}\left(B\left(x^{\prime}, R+r\right) \cap H \cap C\left(\Lambda_{\Gamma}\right)\right) .
$$


Maintenant, si $x^{\prime} \in \mathscr{H} \cap \Omega_{p}$, l'ensemble $B\left(x^{\prime}, R+r\right) \cap H \cap C\left(\Lambda_{\Gamma}\right)$ est inclus dans le $r$-voisinage de $B_{\Omega_{p}}\left(x^{\prime}, R\right) \cap H$ dans $\Omega$. On a donc, d'après le lemme 9.12,

$$
\operatorname{Vol}_{\Omega}\left(B\left(x^{\prime}, R+r\right) \cap H \cap C\left(\Lambda_{\Gamma}\right)\right) \leqslant M \operatorname{Vol}_{\Omega_{p}}\left(A_{r}\right) .
$$

où $A_{r}$ est le $r$-voisinage de $B_{\Omega_{p}}\left(x^{\prime}, R\right) \cap H$ dans $\Omega_{p}$.

La partie de $A_{r}$ qui est dans $H$ correspond précisément à l'intersection

$$
B_{\Omega_{p}}\left(x^{\prime}, R+r\right) \cap H
$$

à laquelle on peut appliquer le lemme 9.5, qui donne :

$$
\operatorname{Vol}_{\Omega_{p}}\left(B_{\Omega_{p}}\left(x^{\prime}, R+r\right) \cap H\right) \leqslant D N_{\mathscr{P}}(x, C(R+r)) .
$$

Le reste de $A_{r}$ est dans un voisinage de taille $r^{\prime}=\max \{r$, diam $\}$ de l'ensemble fini de points $\mathscr{P} \cdot x \cap B(x, R+r)$, où diam est le diamètre d'un domaine fondamental compact pour l'action de $\mathscr{P}$ sur $\mathscr{H} \cap \Omega_{p} \cap C\left(\Lambda_{\Gamma}\right)$ (tout cela pour la distance $d_{\Omega_{p}}$ ). Le volume de cette partie est donc majoré par

$$
N_{\mathscr{P}}\left(x^{\prime}, R+r\right) V_{d}\left(r^{\prime}\right),
$$

où $V_{d}\left(r^{\prime}\right)$ est la constante donnée par le lemme 9.10. Au final, on obtient

$$
\begin{aligned}
\operatorname{Vol}_{\Omega_{p}}\left(A_{r}\right) & \leqslant N_{\mathscr{P}}\left(x^{\prime}, R+r\right) V_{d}\left(r^{\prime}\right)+D N_{\mathscr{P}}\left(x^{\prime}, C(R+r)\right) \\
& \leqslant D^{\prime} N_{\mathscr{P}}\left(x^{\prime}, C(R+r)\right),
\end{aligned}
$$

pour une certaine constante $D^{\prime}$.

En regroupant le tout, on arrive à

$$
\begin{aligned}
\operatorname{Vol}_{\Omega}\left(B(x, R) \cap H \cap C\left(\Lambda_{\Gamma}\right)\right) & \leqslant D^{\prime} N_{\mathscr{P}}\left(x^{\prime}, C(R+r)\right) \\
& \leqslant D^{\prime} N_{\mathscr{P}}(x, C(R+r)+r) .
\end{aligned}
$$

Cela donne le résultat, la condition $R \geqslant 1$ étant due à la présence du $r$.

Le théorème 9.7 soulève la question suivante, sur laquelle nous terminerons ce texte. C'est une question qu'on peut poser de façon très générale mais une réponse dans des cas particuliers serait déjà intéressante.

Question 9.13. Soit $\Omega$ un ouvert proprement convexe de $\mathbb{P}^{n}$ (strictement convexe et à bord $\left.\mathscr{C}^{1}\right)$. A t-on $\delta_{\Gamma}=h_{\mathrm{vol}}\left(C\left(\Lambda_{\Gamma}\right), d_{C\left(\Lambda_{\Gamma}\right)}\right)$ pour tout sous-groupe discret $\Gamma$ de $\operatorname{Aut}(\Omega)$ ? 


\section{Remerciements}

Nous tenons à remercier Françoise Dal'bo pour son intérêt, notamment pour la section 9, ainsi que François Ledrappier qui nous a indiqué l'article [Hamenstädt 1994]. Nous remercions également le rapporteur anonyme pour sa relecture minutieuse et ses remarques pertinentes.

Mickaël Crampon est financé par le programme FONDECYT N 3120071 de la CONICYT (Chile).

\section{Bibliographie}

[Benoist 2000a] Y. Benoist, "Automorphismes des cônes convexes", Invent. Math. 141:1 (2000), 149-193. MR 2001f:22034 Zbl 0957.22008

[Benoist 2000b] Y. Benoist, "Propriétés asymptotiques des groupes linéaires, II", pp. 33-48 dans Analysis on homogeneous spaces and representation theory of Lie groups (Okayama, Kyoto, 1997), édité par T. Kobayashi et al., Adv. Stud. Pure Math. 26, Math. Soc. Japan, Tokyo, 2000. MR 2001e:22005 Zbl 0960.22012

[Benoist 2004] Y. Benoist, "Convexes divisibles, I", pp. 339-374 dans Algebraic groups and arithmetic (Mumbai, 2001), édité par S. G. Dani et G. Prasad, Tata Inst. Fund. Res., Mumbai, 2004. MR 2005h:37073 Zbl 1084.37026

[Benoist 2006] Y. Benoist, "Convexes divisibles, IV: Structure du bord en dimension 3", Invent. Math. 164:2 (2006), 249-278. MR 2007g:22007 Zbl 1107.22006

[Benzécri 1960] J.-P. Benzécri, "Sur les variétés localement affines et localement projectives", Bull. Soc. Math. France 88 (1960), 229-332. MR 23 \#A1325 Zbl 0098.35204

[Colbois et Vernicos 2006] B. Colbois et C. Vernicos, "Bas du spectre et delta-hyperbolicité en géométrie de Hilbert plane”, Bull. Soc. Math. France 134:3 (2006), 357-381. MR 2007g:53080 Zbl 1117.53034

[Cooper et al. 2011] D. Cooper, D. Long et S. Tillmann, "On convex projective manifolds and cusps", preprint, 2011. arXiv 1109.0585

[Coudene et Schapira 2010] Y. Coudene et B. Schapira, "Generic measures for hyperbolic flows on non-compact spaces", Israel J. Math. 179 (2010), 157-172. MR 2012g:37050 Zbl 1229.53078

[Crampon 2009] M. Crampon, "Entropies of strictly convex projective manifolds", J. Mod. Dyn. 3:4 (2009), 511-547. MR 2011g:37079 Zbl 1189.37034

[Crampon 2014] M. Crampon, "Lyapunov exponents in Hilbert geometry", 34:2 (2014), 501-533. arXiv 1105.6275

[Crampon et Marquis 2012] M. Crampon et L. Marquis, "Finitude géométrique en géométrie de Hilbert”, preprint, 2012. À paraître aux Annales de l'Institut Fourier. arXiv 1202.5442

[Crampon et Marquis 2013] M. Crampon et L. Marquis, "Un lemme de Kazhdan-Margulis-Zassenhaus pour les géométries de Hilbert", Ann. Math. Blaise Pascal 20:2 (2013), 363-376. MR 3138033 Zbl 1282.22007

[Dal'bo et al. 2000] F. Dal'bo, J.-P. Otal et M. Peigné, "Séries de Poincaré des groupes géométriquement finis”, Israel J. Math. 118 (2000), 109-124. MR 2001g:37040 
[Dal'Bo et al. 2009] F. Dal'Bo, M. Peigné, J.-C. Picaud et A. Sambusetti, "On the growth of nonuniform lattices in pinched negatively curved manifolds", J. Reine Angew. Math. 627 (2009), 31-52. MR 2010c:53049 Zbl 1184.53039

[Eberlein 1996] P. B. Eberlein, Geometry of nonpositively curved manifolds, University of Chicago Press, 1996. MR 98h:53002 Zbl 0883.53003

[Hamenstädt 1994] U. Hamenstädt, "Anosov flows which are uniformly expanding at periodic points", Ergodic Theory Dynam. Systems 14:2 (1994), 299-304. MR 95c:58132 Zbl 0805.58049

[Katok et Hasselblatt 1995] A. Katok et B. Hasselblatt, Introduction to the modern theory of dynamical systems, Encyclopedia of Mathematics and its Applications 54, Cambridge University Press, 1995. MR 96c:58055 Zbl 0878.58020

Received March 30, 2012.

MICKAËL CRAMPON

UNIVERSIDAD DE SANTIAGo DE CHILE

Departamento de Matemática y Ciencia de la Computación

AVENIDA LAS SOPHORAS 173

Estación Central, SAntiago de Chile

CHILE

mickael.crampon@usach.cl

LUDOVIC MARQUIS

IRMAR

263 AVEnUe du GÉNÉRAL LECLERC, CS 74205

35042 RENNES CÉDEX

FRANCE

ludovic.marquis@univ-rennes1.fr 


\title{
PACIFIC JOURNAL OF MATHEMATICS
}

\author{
msp.org/pjm
}

Founded in 1951 by E. F. Beckenbach (1906-1982) and F. Wolf (1904-1989)

\section{EDITORS}

Don Blasius (Managing Editor)

Department of Mathematics

University of California

Los Angeles, CA 90095-1555

blasius@math.ucla.edu

\author{
Paul Balmer \\ Department of Mathematics \\ University of California \\ Los Angeles, CA 90095-1555 \\ balmer@math.ucla.edu \\ Robert Finn \\ Department of Mathematics \\ Stanford University \\ Stanford, CA 94305-2125 \\ finn@math.stanford.edu \\ Sorin Popa \\ Department of Mathematics \\ University of California \\ Los Angeles, CA 90095-1555 \\ popa@math.ucla.edu
}

\author{
Vyjayanthi Chari \\ Department of Mathematics \\ University of California \\ Riverside, CA 92521-0135 \\ chari@math.ucr.edu \\ Kefeng Liu \\ Department of Mathematics \\ University of California \\ Los Angeles, CA 90095-1555 \\ liu@math.ucla.edu \\ Jie Qing \\ Department of Mathematics \\ University of California \\ Santa Cruz, CA 95064 \\ qing@ cats.ucsc.edu
}

\section{PRODUCTION}

Silvio Levy, Scientific Editor, production@msp.org

\section{SUPPORTING INSTITUTIONS}

ACADEMIA SINICA, TAIPEI

CALIFORNIA INST. OF TECHNOLOGY

INST. DE MATEMÁTICA PURA E APLICADA

KEIO UNIVERSITY

MATH. SCIENCES RESEARCH INSTITUTE

NEW MEXICO STATE UNIV.

OREGON STATE UNIV.

\author{
STANFORD UNIVERSITY \\ UNIV. OF BRITISH COLUMBIA \\ UNIV. OF CALIFORNIA, BERKELEY \\ UNIV. OF CALIFORNIA, DAVIS \\ UNIV. OF CALIFORNIA, LOS ANGELES \\ UNIV. OF CALIFORNIA, RIVERSIDE \\ UNIV. OF CALIFORNIA, SAN DIEGO \\ UNIV. OF CALIF., SANTA BARBARA
}

\author{
Daryl Cooper \\ Department of Mathematics \\ University of California \\ Santa Barbara, CA 93106-3080 \\ cooper@math.ucsb.edu \\ Jiang-Hua Lu \\ Department of Mathematics \\ The University of Hong Kong \\ Pokfulam Rd., Hong Kong \\ jhlu@maths.hku.hk \\ Paul Yang \\ Department of Mathematics \\ Princeton University \\ Princeton NJ 08544-1000 \\ yang@math.princeton.edu
}

These supporting institutions contribute to the cost of publication of this Journal, but they are not owners or publishers and have no responsibility for its contents or policies.

See inside back cover or msp.org/pjm for submission instructions.

The subscription price for 2014 is US $\$ 410 /$ year for the electronic version, and \$535/year for print and electronic.

Subscriptions, requests for back issues and changes of subscribers address should be sent to Pacific Journal of Mathematics, P.O. Box 4163, Berkeley, CA 94704-0163, U.S.A. The Pacific Journal of Mathematics is indexed by Mathematical Reviews, Zentralblatt MATH, PASCAL CNRS Index, Referativnyi Zhurnal, Current Mathematical Publications and Web of Knowledge (Science Citation Index).

The Pacific Journal of Mathematics (ISSN 0030-8730) at the University of California, c/o Department of Mathematics, 798 Evans Hall \#3840, Berkeley, CA 94720-3840, is published twelve times a year. Periodical rate postage paid at Berkeley, CA 94704, and additional mailing offices. POSTMASTER: send address changes to Pacific Journal of Mathematics, P.O. Box 4163, Berkeley, CA 94704-0163.

PJM peer review and production are managed by EditFLOW ${ }^{\circledR}$ from Mathematical Sciences Publishers.

\section{PUBLISHED BY}

\section{mathematical sciences publishers \\ nonprofit scientific publishing}

http://msp.org/

(C) 2014 Mathematical Sciences Publishers 


\title{
PACIFIC JOURNAL OF MATHEMATICS
}

\author{
Volume $268 \quad$ No. $2 \quad$ April 2014
}

In memoriam: Jonathan Rogawski

DON BLASIUS, DINAKAR RAMAKRISHNAN and V. S. VARADARAJAN

Formes modulaires sur la $\mathbb{Z}_{p}$-extension cyclotomique de $\mathbb{Q}$

LAURENT CLOZEL

Weight zero Eisenstein cohomology of Shimura varieties via Berkovich

275 spaces

MICHAEL HARRIS

$\Lambda$-adic Barsotti-Tate groups

HARUZO HIDA

Le flot géodésique des quotients géométriquement finis des géométries de

Hilbert

MickaËl CRAMPON and LUdOViC MARQuis

Nonplanarity of unit graphs and classification of the toroidal ones

A. K. Das, H. R. Maimani, M. R. Pournaki and S. Yassemi

Discrete semiclassical orthogonal polynomials of class one

DIEGO DOMINICI and FRANCISCO MARCELLÁN

A note on conformal Ricci flow

PENG LU, Jie QING and Yu Zheng

On representations of $\mathrm{GL}_{2 n}(F)$ with a symplectic period

ARNAB Mitra

Linked triples of quaternion algebras

ALEXANDER S. SIVATSKI

Finite nonsolvable groups with many distinct character degrees

Hung P. TONG-VIET

Errata to "Dynamics of asymptotically hyperbolic manifolds"

JULIE ROWLETT

Erratum to "Singularities of the projective dual variety" 